\title{
A model-based iterative reconstruction algorithm DIRA using patient-specific tissue classification via DECT for improved quantitative $\mathrm{CT}$ in dose planning
}

Alexandr Malusek, Maria Magnusson, Michael Sandborg and Gudrun Alm Carlsson

The self-archived postprint version of this journal article is available at Linköping University Institutional Repository (DiVA):

http:/ / urn.kb.se/ resolve?urn=urn:nbn:se:liu:diva-140809

N.B.: When citing this work, cite the original publication.

Malusek, A., Magnusson, M., Sandborg, M., Alm Carlsson, G., (2017), A model-based iterative reconstruction algorithm DIRA using patient-specific tissue classification via DECT for improved quantitative CT in dose planning, Medical physics (Lancaster), 44(6), 2345-2357.

https:// doi.org/ 10.1002/ mp.12238

Original publication available at:

https:/ / doi.org/ 10.1002/ mp.12238

Copyright: American Association of Physicists in Medicine (Medical Physics)

http:// www.aapm.org/ main.asp 


\title{
A model-based iterative reconstruction algorithm DIRA using patient-specific tissue classification via DECT for improved quantitative CT in dose planning
}

\author{
Alexandr Malusek* \\ Radiation Physics, Department of Medical and Health Sciences, Linköping University, Linköping, Sweden, \\ and Center for Medical Image Science and Visualization (CMIV), Linköping University, Linköping, Sweden \\ Maria Magnusson ${ }^{\dagger}$ \\ Computer Vision Laboratory, Department of Electrical Engineering, \\ Linköping University, Linköping, Sweden; Radiation Physics, \\ Department of Medical and Health Sciences, Linköping University, Linköping, Sweden, \\ and Center for Medical Image Science and Visualization (CMIV), Linköping University, Linköping, Sweden \\ Michael Sandborg \\ Radiation Physics, Department of Medical and Health Sciences, Linköping University, Linköping, Sweden, \\ and Center for Medical Image Science and Visualization (CMIV), Linköping University, Linköping, Sweden \\ Gudrun Alm Carlsson \\ Radiation Physics, Department of Medical and Health Sciences, Linköping University, Linköping, Sweden, \\ and Center for Medical Image Science and Visualization (CMIV), Linköping University, Linköping, Sweden
}

(Dated: October 27, 2017)

\begin{abstract}
Purpose: To develop and evaluate - in a proof-of-concept configuration - a novel iterative reconstruction algorithm (DIRA) for quantitative determination of elemental composition of patient tissues for application to brachytherapy with low energy $(<50 \mathrm{keV})$ photons and proton therapy. Methods: DIRA was designed as a model-based iterative reconstruction algorithm, which uses filtered backprojection, automatic segmentation and multimaterial tissue decomposition. The evaluation was done for a phantom derived from the voxelized ICRP 110 male phantom. Soft tissues were decomposed to the lipid, protein and water triplet, bones were decomposed to the compact bone and bone marrow doublet. Projections were derived using the Drasim simulation code for an axial scanning configuration resembling a typical DECT (dual-energy CT) scanner with $80 \mathrm{kV}$ and $\mathrm{Sn} 140 \mathrm{kV}$ x-ray spectra. The iterative loop produced mono-energetic images at 50 and $88 \mathrm{keV}$ without beam hardening artifacts. Different noise levels were considered: no noise, a typical noise level in diagnostic imaging and reduced noise level corresponding to tenfold higher doses. An uncertainty analysis of the results was performed using type A and B evaluations. The two approaches were compared.

Results: Linear attenuation coefficients averaged over a region were obtained with relative errors less than $0.5 \%$ for all evaluated regions. Errors in average mass fractions of the three-material decomposition were less than 0.04 for no noise and reduced noise levels and less than 0.11 for the typical noise level. Mass fractions of individual pixels were strongly affected by noise, which slightly increased after the first iteration but subsequently stabilized. Estimates of uncertainties in mass fractions provided by the type B evaluation differed from the type A estimates by less than $1.5 \%$ for most cases. The algorithm was fast, the results converged after 5 iterations. The algorithmic complexity of forward polyenergetic projection calculation was much reduced by using material doublets and triplets.

Conclusions: The simulations indicated that DIRA is capable of determining elemental composition of tissues, which are needed in brachytherapy with low energy $(<50 \mathrm{keV})$ photons and proton therapy. The algorithm provided quantitative monoenergetic images with beam hardening artifacts removed. Its convergence was fast, image sharpness expressed via the modulation transfer function was maintained, and image noise did not increase with the number of iterations.
\end{abstract}

\section{INTRODUCTION}

$\mathrm{X}$-ray CT is the basis for dose calculations in radiotherapy. In external beam therapy with MV photon beams,

\footnotetext{
* Alexandr.Malusek@liu.se

$\dagger$ A. Malusek and M. Magnusson have made equal intellectual contributions to the manuscript and the associated scientific investigation
}

electron density is the quantity needed for accurate dose calculations because Compton scattering is the dominant interaction process at these high photon energies. With development of new applications for brachytherapy with low energy $(<50 \mathrm{keV})$ photons and proton therapy, information on electron densities is not sufficient. Knowledge of the elemental composition (concentrations of $\mathrm{H}$, $\mathrm{C}, \mathrm{N}, \mathrm{O}$ etc) of the tissues is also needed [1]. This is because in brachytherapy with low energy photons, the photoelectric effect is an important interaction process 
that depends strongly on atomic number.

Until now, dose calculations for treatment planning in brachytherapy have been done assuming soft tissues to be equivalent to water [2]. This may lead to errors in absorbed doses to soft tissues of up to a factor of 2 using 20-30 keV photons as emitted from radioactive seeds of, e.g., ${ }^{125} \mathrm{I}$ and ${ }^{103} \mathrm{Pd}$ and miniature $\mathrm{x}$-ray sources operated at $50 \mathrm{kV}$ (Figure 3 in Ref. 1, based on data in Ref. 3). For accurate treatment planning the soft tissues need, in these instances, be known individually on a voxel by voxel basis [1]. Model based dose calculation algorithms (MBDCAs) taking into account patient anatomy [1] have recently been developed and implemented in commercially available treatment planning systems, viz., Electa's "Oncentra Brachy" and Varian's "Brachy Vision". Accurate tools providing information on the elemental composition of the tissues is, however, still not available [1]. In radiotherapy with protons and heavier ions, mean excitation potentials ( $I$-values) and nuclear cross sections for these particles depend on atomic number [4]. Significant errors in $I$-values increase range uncertainties and consequently the position of the Bragg peak [5]; relative concentrations of $\mathrm{C}$ and $\mathrm{O}$ are particularly important [6]. As in brachytherapy, there is need for accurate tools for determination of elemental compositions. A recent review [7] on dual energy $\mathrm{CT}$ in radiotherapy summarizes the needs and status for both modalities.

In contemporary CT scanners, beam hardening and scatter artifacts adversely affect the accuracy of quantitative $\mathrm{CT}$ (QCT). In principle, the artifacts could be removed by model-based iterative reconstruction (MBIR) methods [8], which include geometric modeling, physical modeling and prior object information modeling. For QCT, modeling of the poly-energetic x-ray spectrum and material composition is most important, but only few reconstruction algorithms described in scientific literature deal with both. For instance the iterative maximum-likelihood polychromatic algorithm for CT [9] and the MBIR algorithm for QCT [10] (a precursor to the algorithm presented in this article) modeled the polyenergetic x-ray spectrum and material decomposition of body tissues in single- and dual-energy CT, respectively. Iterative reconstruction algorithms implemented in commercially available CT scanners (e.g., ADMIRE, ASiR$\mathrm{V}$ ) implement some of the MBIR techniques (details have not been published in scientific literature), but they primarily focus on noise reduction so as to allow reduction of patient doses while maintaining (or improving) image quality for diagnostic purposes; they are not optimized for QCT.

Material composition of the imaged object can be modeled by material decomposition, i.e. a method determining fractions of pre-selected material components (bases) using projection-space or image-space data. In projection space, a decomposition to (i) photoelectric effect and Compton scattering components or (ii) two arbitrary material bases, e.g. water and bone, can be done inside a non-iterative image reconstruction algorithm in DECT described by Alvarez and Macovsky [11]. The algorithm suppresses the beam hardening artifact, but the resulting CT values are biased since coherent scattering is not considered in the former case and any two bases cannot fully represent all body tissues in the latter case. The algorithm demands geometrically consistent raw data projections.

Several methods performing the material decomposition in image space have been developed. The material decomposition from inconsistent rays (MDIR) [12] is an iterative algorithm that can be used when geometrically consistent raw data projections are not available. Lower noise and bias than in the MDIR method can be achieved in the extended algebraic reconstruction technique (EART) [13] at the expense of longer computation time. The iterative reconstruction algorithm for polychromatic CT imaging [14] (here abbreviated as RAPCTI) can suppress beam hardening artifact in single-energy CT and, in case of DECT, also allows material decomposition to two material bases.

Material decomposition in image space performed on already reconstructed data was used in the following algorithms. A two-material decomposition was applied in single-energy CT [15] and a three-material decomposition in DECT. For instance DECT was used to quantify mass fractions of water, hydroxyapatite and aqueous iron nitrate[16]. The method was also applied for the determination of (i) iron content in liver composed of soft tissue, fat and iron (ii) bone mineral density in a trabecular bone composed of calcium hydroxyapatite, yellowand read-marrow[17]. The three-material decomposition method in DECT was extended to a framework that also includes the two-material decomposition and tissue segmentation to quantify soft tissues in terms of their mass fractions of water, protein and lipid[18]. Another approach using image data from DECT is to determine electron densities and effective atomic numbers of the materials [19-21]. Additional mathematical models that link these quantities to elemental concentrations $[6,22]$ or $I$-values [23] have been developed. However, all the non-iterative image-space based methods cited above use data from commercially available CT scanners and are prone to inaccuracies due to biased CT numbers caused by beam hardening.

Today's radiation therapy requires quantitative information about tissue compositions, a need that current commercial image reconstruction algorithms do not satisfy. To address this problem the aim of this work was to develop and evaluate the MBIR dual-energy iterative reconstruction algorithm (DIRA), whose design goals are: (i) Conversion of polyenergetic reconstruction results to monoenergetic and thereby the elimination of beam hardening artifacts, and (ii) the decomposition of human tissues in doublets and triplets of base materials.

In this work a proof of concept is presented for the determination of material composition using the DIRA algorithm with simulated data calculated for anthropomorphic phantoms. As the accuracy and precision of ma- 
terial decomposition depends on noise in projection data, the behavior of the algorithm is presented and evaluated for three different noise levels and an analysis of associated uncertainties is performed.

\section{THEORY}

\section{A. Three-material decomposition in DECT}

The system of equations in the three-material decomposition method (3MD) in DECT can be derived from (i) the summation rule

$$
\mu_{\mathrm{m}}(E)=\sum_{i=1}^{3} w_{i} \mu_{\mathrm{m}, i}(E),
$$

where $\mu_{\mathrm{m}}(E)$ is the mass attenuation coefficient of a mixture at photon energy $E$ and $\mu_{\mathrm{m}, i}(E)$ is the mass attenuation coefficient at the same energy $E$ of the $i$ th base material with the mass fraction $w_{i}(1 \leq i \leq 3)$, (ii) the normalization condition $w_{1}+w_{2}+w_{3}=1$, and (iii) the formula for the mass density of a mixture

$$
\frac{1}{\rho}=\sum_{i=1}^{3} \frac{w_{i}}{\rho_{i}}
$$

where $\rho$ and $\rho_{i}$ are mass densities of the mixture and $i$ th material, respectively. Formula (2) is valid when partial molar volumes of base materials are the same when the materials are standalone and in the mixture. Eqs. (1) and (2) and the normalization condition lead to a system of linear equations that can be written in the matrix form as

$$
\mathbf{A w}=\mathbf{b}
$$

where $\mathbf{b}=(0,0,1)^{T}$ is the right-hand side vector, $\mathbf{w}=$ $\left(w_{1}, w_{2}, w_{3}\right)^{T}$ is the vector of unknown mass fractions and $\mathbf{A}$ is the system matrix. The superscript $T$ denotes a matrix transpose; the vectors $\mathbf{b}$ and $\mathbf{w}$ are represented by $3 \times 1$ matrices. The elements of the system matrix are

$$
A_{i, j}=\left\{\begin{array}{ll}
\frac{\mu\left(E_{i}\right)}{\rho_{j}}-\mu_{\mathrm{m}, j}\left(E_{i}\right) & i=1,2 \\
1 & i=3
\end{array},\right.
$$

where $j=1,2,3$ and $\mu=\mu_{\mathrm{m}} \rho$ is the linear attenuation coefficient. The mass fractions can be obtained as

$$
\mathbf{w}=\mathbf{A}^{-1} \mathbf{b},
$$

where $\mathbf{A}^{-1}$ is the inverse matrix of $\mathbf{A}$ in Eq. (4).

\section{B. Two-material decomposition in DECT}

In the two-material decomposition method (2MD) in DECT [18], the summation rule (1) and the normalization condition $w_{1}+w_{2}=1$ lead to a system of equations that can be written in the matrix form as

$$
\left(\begin{array}{ccc}
\mu_{\mathrm{m}, 1}\left(E_{1}\right) & \mu_{\mathrm{m}, 2}\left(E_{1}\right) & -\mu\left(E_{1}\right) \\
\mu_{\mathrm{m}, 1}\left(E_{2}\right) & \mu_{\mathrm{m}, 2}\left(E_{2}\right) & -\mu\left(E_{2}\right) \\
1 & 1 & 0
\end{array}\right)\left(\begin{array}{c}
w_{1} \\
w_{2} \\
1 / \rho
\end{array}\right)=\left(\begin{array}{l}
0 \\
0 \\
1
\end{array}\right),
$$

where the mass fractions $w_{1}$ and $w_{2}$ and the mass density $\rho$ of the mixture are unknown, the linear attenuation coefficients $\mu\left(E_{1}\right)$ and $\mu\left(E_{2}\right)$ of the mixture are known. Eq. (6) can be solved as

$$
\mathbf{x}=\mathbf{A}^{-1} \mathbf{b}
$$

where $\mathbf{x}=\left(w_{1}, w_{2}, 1 / \rho\right)^{T}$ is the unknown vector, $\mathbf{b}=$ $(0,0,1)^{T}$ is the right-hand side vector, and $\mathbf{A}$ is the system matrix in Eq. (6).

\section{DIRA}

The model-based iterative image reconstruction algorithm DIRA uses CT projections obtained at two different tube voltages and automatic quantitative tissue classification for the generation of the reconstructed volume. The main idea is that polyenergetic projections measured by a CT scanner are converted to monoenergetic ones by adding a correction term to each measured projection. The correction terms are calculated by simulating monoenergetic and polyenergetic projections using the reconstructed volume for each of the used tube voltages. The algorithm is illustrated in Fig. 1.

Step 1: Measured polyenergetic projections for two tube voltages are obtained. At iteration 0 and step 2, classical water beam-hardening correction[24, p. 121] (WBHC) is performed, otherwise not. Step 3: Reconstruction with filtered backprojection (FBP) is performed and reconstructed images $\mu_{1}$ and $\mu_{2}$ are obtained. These are then automatically segmented into pre-defined tissues and organs at step 4 . In the presented example, a threshold segmentation to soft and bone tissue combined with filling of bone cavities is used. The user is free to choose different segmentation methods. In step 5 , classification into mass fractions of base materials is performed. For soft tissue, three-material decomposition (3MD) (Section IIA) is used and for bone tissue two-material decomposition (2MD) (Section IIB) is applied. Forward monoenergetic and polyenergetic projections are calculated in steps 6 and 7 . The goal is that after some iterations, the calculated polyenergetic projections become almost equal to the measured polyenergetic projections. Then only the calculated monoenergetic projections contribute to the reconstructed $\mu$-volumes. The filter functions $W_{0}$, $W_{\mathrm{A}}$ and $W_{\mathrm{B}}$ are described in Supplementary material. The result of DIRA is the classified reconstructed volume (CRV) in step 8.

To formulate DIRA in mathematical terms, set

$$
\boldsymbol{\mu}=\left(\begin{array}{c}
\mu_{1} \\
\mu_{2}
\end{array}\right), \quad \mathbf{P}_{\mathbf{M}, \mathbf{U}}=\left(\begin{array}{c}
P_{M, U_{1}} \\
P_{M, U_{2}}
\end{array}\right),
$$




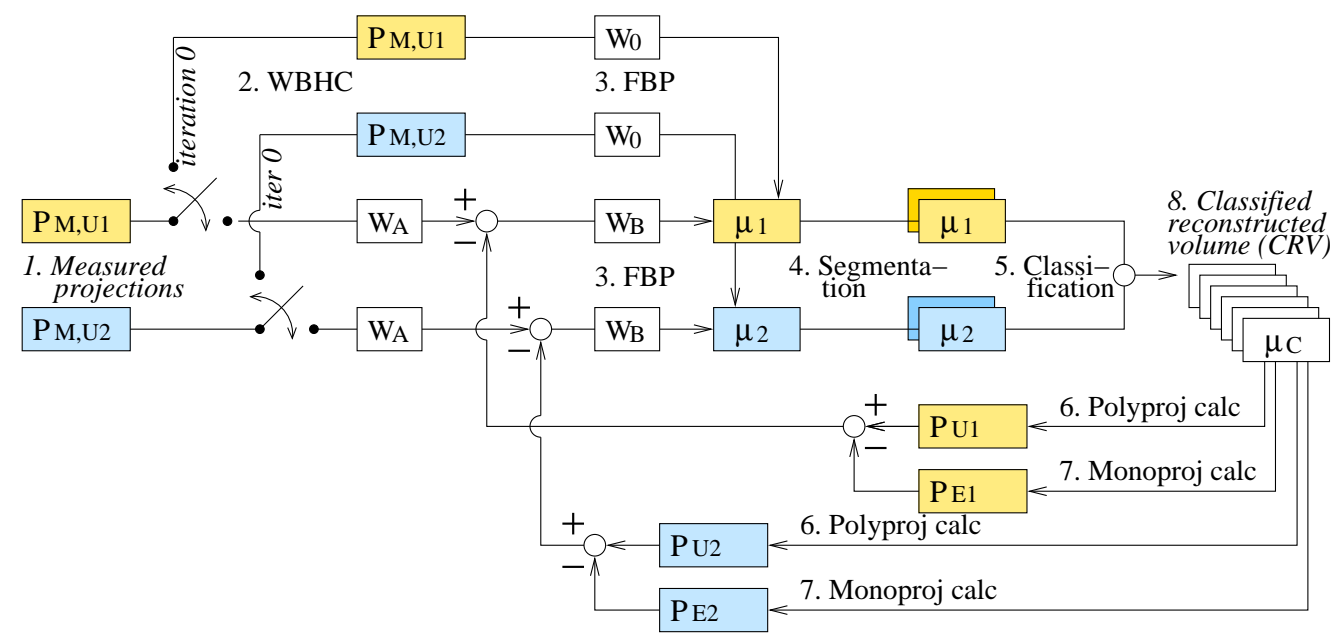

FIG. 1. Data-flowchart of the dual-energy iterative reconstruction algorithm DIRA. $P_{\mathrm{M}, \mathrm{U}_{1}}$ and $P_{\mathrm{M}, \mathrm{U}_{2}}$ denote measured polyenergetic projections at tube voltages $U_{1}$ and $U_{2}$, respectively. $P_{\mathrm{U}_{1}}$ and $P_{\mathrm{E}_{1}}$ denote simulated polyenergetic and monoenergetic projections at the tube voltage $U_{1}$ and effective energy $E_{1}$ (see section IID), respectively, and similarly for $P_{\mathrm{U}_{2}}$ and $P_{\mathrm{E}_{2}}$. The linear attenuation coefficients $\mu_{1}$ and $\mu_{2}$ denote reconstructed images. $W_{0}, W_{\mathrm{A}}$, and $W_{\mathrm{B}}$ denote filter functions (windows).

for the reconstructed images and the measured projections (sinograms), respectively. Furthermore, denote the filtered backprojection operator $\mathcal{B}$, and the filtering operator $\mathcal{F}_{W_{k}}$, where $k=0, A, B$ denotes different filter functions. Set

$$
\mathcal{P}_{U}=\left(\begin{array}{c}
\mathcal{P}_{U_{1}} \\
\mathcal{P}_{U_{2}}
\end{array}\right), \quad \mathcal{P}_{E}=\left(\begin{array}{c}
\mathcal{P}_{E_{1}} \\
\mathcal{P}_{E_{2}}
\end{array}\right)
$$

for the projection operator for polyenergetic projections and monoenergetic projections, respectively. These projection operators include the automatic tissue segmentation and classification. The linear attenuation coefficient $\boldsymbol{\mu}^{(i+1)}$ obtained at the $(i+1)$ th iteration is

$$
\begin{aligned}
\boldsymbol{\mu}^{(i+1)} & =\mathcal{B F}_{W_{A}} \mathcal{F}_{W_{B}}\left(\mathbf{P}_{\mathbf{M}, \mathbf{U}}\right)-\mathcal{B F}_{W_{B}} \mathcal{P}_{U}\left(\boldsymbol{\mu}^{(i)}\right) \\
& +\mathcal{B} \mathcal{F}_{W_{B}} \mathcal{P}_{E}\left(\boldsymbol{\mu}^{(i)}\right),
\end{aligned}
$$

The filter function $W_{0}$ contributes multiplicatively to the modulation transfer function (MTF) of the imaging system at iteration 0 of the algorithm, and the product $W_{A} \cdot W_{B}$ contributes multiplicatively to the MTF at iteration $i$. In the presented work, $W_{A}=1$ for reasons discussed in Supplementary material.

Assume that $W_{A}=W_{B}=W_{0}=1$, i.e. disregard the filters. Eq. (10) then reduces to

$$
\boldsymbol{\mu}^{(i+1)}=\mathcal{B}\left(\mathbf{P}_{\mathbf{M}, \mathbf{U}}\right)-\mathcal{B} \mathcal{P}_{U}\left(\boldsymbol{\mu}^{(i)}\right)+\mathcal{B} \mathcal{P}_{E}\left(\boldsymbol{\mu}^{(i)}\right) .
$$

Ideally, the simulated polyenergetic projections $\mathcal{P}_{U}\left(\boldsymbol{\mu}^{(i)}\right)$ converge towards the measured projection $\mathbf{P}_{\mathbf{M}, \mathbf{U}}$. The $(i+1)$ th iteration then gives $\boldsymbol{\mu}^{(i+1)} \approx \mathcal{B} \mathcal{P}_{E}\left(\boldsymbol{\mu}^{(i)}\right)$, which is the filtered backprojection result of the monoenergetic projections.

Eq. (11) can be rewritten to

$$
\boldsymbol{\mu}^{(i+1)}=\mathcal{B}\left(\mathbf{P}_{\mathbf{M}, \mathbf{U}}\right)-\mathcal{B} \mathcal{P}_{U}\left(\boldsymbol{\mu}^{(i)}\right)+\boldsymbol{\mu}^{(i)}-\mathcal{F}_{H P}\left(\boldsymbol{\mu}^{(i)}(12)\right.
$$

where $\mathcal{F}_{H P}\left(\boldsymbol{\mu}^{(i)}\right)=\boldsymbol{\mu}^{(i)}-\mathcal{B} \mathcal{P}_{E}\left(\boldsymbol{\mu}^{(i)}\right)$ denotes the result of filtration with a high pass filter. The reason is that projection followed by backprojection, $\mathcal{B P}_{E}$, serves as a low pass filter on the original function. Eq. (12) shows that the generation of monoenergetic projections followed by backprojection in DIRA serve as a regularization. The importance of regularization stems from the fact that non-regularized iterative methods tend to increase noise in reconstructed images, more information is in section V.

The polyenergetic spectra and the tissue classification in base materials complicate the proof of convergence of Eqs. (10) and (11). With no proof available, the applicability of the method is shown experimentally by realistic simulations.

\section{Computation of projections in DIRA}

For a polyenergetic spectrum, the x-ray beam intensity at the detector position is given by

$$
I=\int_{0}^{E_{\max }} E N(E) \exp \left[-\int_{L} \mu(x, y, E) \mathrm{d} l\right] \mathrm{d} E,
$$

where $E N(E) \mathrm{d} E$ is the energy carried by $N(E) \mathrm{d} E$ photons with energies in the interval $(E, E+\mathrm{d} E), \mu(x, y, E)$ is the linear attenuation coefficient of the object at position $(x, y)$ for photons with energy $E$. The inner integration is performed over a straight line $L$ through the object. For an unattenuated ray that does not intersect the object, we get

$$
I_{0}=\int_{0}^{E_{\max }} E N(E) \mathrm{d} E .
$$


Projection data is calculated by

$$
P=\ln \left(\frac{I_{0}}{I}\right) .
$$

For a monoenergetic spectrum, $P$ is equal to the line integral of the object function $\int_{L} \mu(x, y, E) \mathrm{d} l$. For the sake of simplicity an ideal energy integrating detector is assumed. A generalization to a detector with a known energy-dependent efficiency is straightforward.

As mentioned above, the reconstructed object is segmented into tissues defined by the user, for instance bone and soft tissue. Each tissue is then decomposed using the $2 \mathrm{MD}$ or $3 \mathrm{MD}$. For the sake of brevity, the $3 \mathrm{MD}$ is used in the following explanation, i.e each voxel $\mu(x, y, E)$ is classified as a mixture of three base materials with mass attenuation coefficients $\mu_{\mathrm{m}, i}(E), i=1,2,3$ and mass fractions $w_{i}$ corresponding to Eq. (1). A modification for the $2 \mathrm{MD}$ is straightforward. The Eq. (13) can be written with $\mu$ replaced with $\rho \mu_{\mathrm{m}}$, where $\rho$ is the density of the mixture. This density is not known and so an approximation defined by Eq. (2) was made. Using Eq. (1), Eq. (13) can be written as

$$
\begin{aligned}
I & =\int_{0}^{E_{\max }} E N(E) \\
& \times \exp \left\{-\int_{L}\left[\rho(x, y) \sum_{i=1}^{3} \mu_{\mathrm{m}, i}(E) w_{i}(x, y)\right] \mathrm{d} l\right\} \mathrm{d} E \\
& =\int_{0}^{E_{\max }} E N(E) \\
& \times \exp \left[-\sum_{i=1}^{3} \mu_{i}(E) \rho_{i}^{-1} \int_{L} \rho(x, y) w_{i}(x, y) \mathrm{d} l\right] \mathrm{d} E(1 \\
& =\int_{0}^{E_{\max }} E N(E) \exp \left[-\sum_{i=1}^{3} \mu_{i}(E) l_{i}\right] \mathrm{d} E
\end{aligned}
$$

where $\rho_{i}$ and $\mu_{i}(E)$ are the mass density and linear attenuation coefficient, respectively, of the $i$ th base material and

$$
l_{i}=\rho_{i}^{-1} \int_{L} \rho(x, y) w_{i}(x, y) \mathrm{d} l=\rho_{i}^{-1} \int_{L} \rho_{\mathrm{p}, i}(x, y) \mathrm{d} l
$$

is the line integral of the partial mass density $\rho_{\mathrm{p}, i}$ normalized per unit mass density $\rho_{i}$ of the $i$ th base material. (The partial mass density is the mass of the $i$ th base material in a unit volume of the mixture.) Such line integrals are calculated with Joseph's method [25].

Eqs. (18) and (19) show that the calculation of line integrals can be performed on the three images of partial mass densities $\rho_{\mathrm{p}, i}$. The influence of the X-ray spectrum and linear attenuation coefficients can be taken into account afterwards. The intensity spectrum of the source $E N(E)$, and the linear attenuation coefficients as functions of energy $\mu_{i}(E)$, can be re-sampled to a common grid $E_{k}, k=0, . ., K$. Then Eq. (18) can be computed using the Simpson's formula

$$
I \approx \frac{1}{2} \sum_{k=1}^{K-1} E_{k} N\left(E_{k}\right) \exp \left[-\sum_{i=1}^{3} \mu_{i}\left(E_{k}\right) l_{i}\right]\left(E_{k+1}-E_{k-1}\right) .
$$

Finally, the polyenergetic projections are received by inserting $I$ into (15). Direct computation of Eq. (17) is considerably faster than computation of Eq. (16). The corresponding time complexities (see Supplementary material) are $O(K N)$ and $O(K+N)$ for discretized formulas in Eqs. (16) and (17), respectively, where $K$ is the number of energy bins and $N \times N$ is the size of the voxel array.

Monoenergetic projections for the energies $E_{j}, j=1,2$, are calculated as

$$
P_{E_{j}}=\sum_{i=1}^{3} \mu_{i}\left(E_{j}\right) l_{i}
$$

where the summation is done over all three base materials.

\section{METHODS}

\section{A. The phantom}

An anthropomorphic phantom was created by approximating three slices (slice numbers 111, 113 and 115) of the ICRP 110 voxel male phantom [26] with ellipses fitting the tissue structures, see Fig. 2. The approximation was done to overcome the limitation of the Drasim code [27], which cannot calculate CT projections of voxel phantoms. The prostate-containing slices demonstrate large variations in the shape and positions of pelvic bones, which cause strong beam hardening artifacts in conventional CT. The resulting mathematical model for slice B is shown in Fig. 3. Elemental composition and mass density of materials in the three slices (adipose tissue (49), muscle (29), pelvis spongiosa (14), femora spongiosa (9), mineral bone (2), prostate (46), and urinary bladder (41)) were taken from Ref. 26; the corresponding tissue numbers are given in parenthesis. Linear attenuation coefficients of the tissues are shown in Fig. 4.

\section{B. CT scanner simulation}

The scanner geometry is described in Supplementary material. The energy $E_{j}, j=1,2$, in Eq. (21), here referred to as the effective energy, was chosen as the energy for which the linear attenuation coefficient for water equals the energy fluence weighted mean linear attenuation coefficient for water. The weighting was done for the energy spectrum of photons emitted from the $\mathrm{x}$ ray source. In this work, effective energies of 50 and 

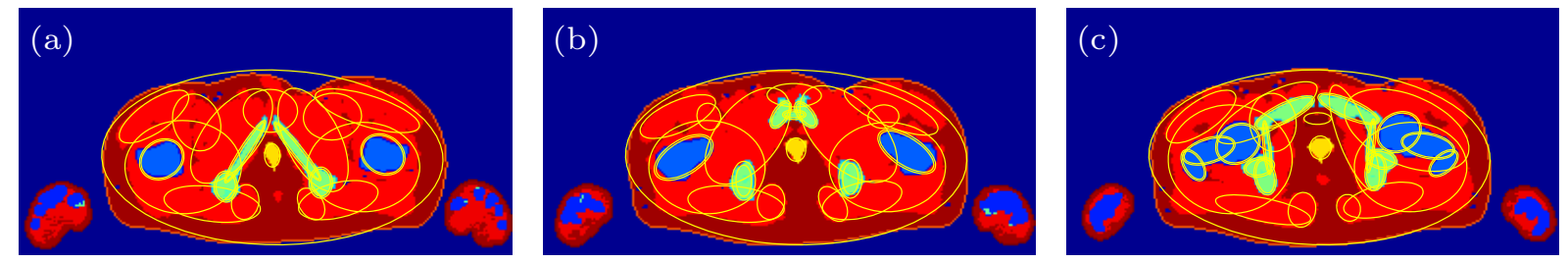

FIG. 2. A colormap of material numbers in transversal slices A (a), B (b) and C (c) of the pelvic region of the ICRP 110 voxel phantom. Ellipses approximating tissue boundaries were used to construct mathematical models of the slices.

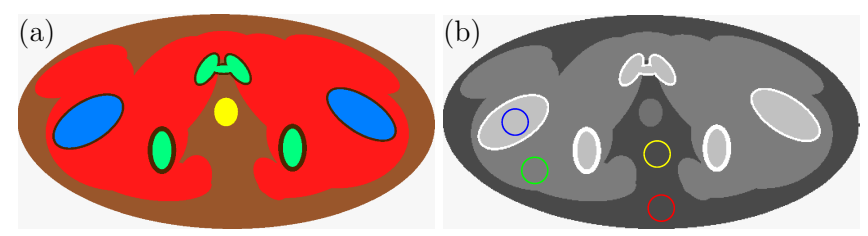

FIG. 3. (a) Color-coding of the resulting mathematical model of slice B: adipose tissue (brown), muscle (red), pelvis spongiosa (green), femora spongiosa (blue), mineral bone (black), prostate (yellow). (b) Circular regions for statistical analysis in slice B: $R_{\mathrm{M}}=$ muscle (green), $R_{\mathrm{A}}=$ peripheral adipose tissue (red), $R_{\mathrm{C}}=$ central adipose tissue (yellow), $R_{\mathrm{F}}=$ femora spongiosa (blue).

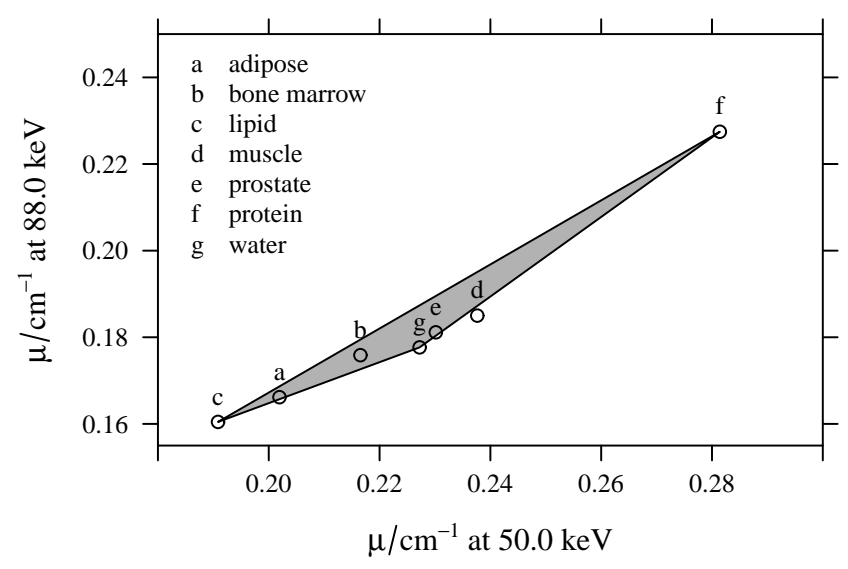

FIG. 4. Position of the chosen lipid, protein, and water triplet in the linear attenuation diagram. The point for mineral bone $\left(0.79 \mathrm{~cm}^{-1}, 0.39 \mathrm{~cm}^{-1}\right)$ is off the scale.

$88 \mathrm{keV}$ were used for the $80 \mathrm{kV}$ and $\mathrm{Sn} 140 \mathrm{kV}$ source energy spectra, respectively. Other approaches are possible, the algorithm is not very sensitive to the settings of $E_{j}, j=1,2$.

Simulations were performed for three levels of quantum noise in reconstructed images. The level 2 corresponded to a typical noise in clinical CT images (see Supplementary material). In this case, tube loads for a single projection were set to 0.217 and $0.080 \mathrm{mAs}$ for the low and high tube voltages, respectively, in Drasim. This corresponded to tube loads of $0.217 \cdot 1152=250 \mathrm{mAs}$ and $0.080 \cdot 1152=92.16 \mathrm{mAs}$ per full rotation of the gantry for the two tube voltages. The level 1 corresponded to tube loads increased by a factor of 10 compared to the level 2. The level 0 corresponded to no quantum noise. The tube load was the same for all projections; tube current modulation was not simulated.

\section{Tissue segmentation}

The reconstructed image at $50 \mathrm{keV}$ was segmented to outside air (pixels with $\mu<19 \mathrm{~m}^{-1}$ ), soft tissue (pixels with $19 \mathrm{~m}^{-1} \leq \mu \leq 33 \mathrm{~m}^{-1}$ ) and bone (pixels with $33 \mathrm{~m}^{-1}<\mu$ ) using threshold segmentation. If these values are outside of reasonable limits, the computed segmentation masks may not be accurate. The computation of the linear attenuation coefficients is not, however, affected much by those inaccuracies in the segmentation. Pixels at tissue boundaries received special treatment to reduce the adverse effect of volume averaging on the material decomposition routines: (i) The air-soft tissue boundary pixels were assigned to air by setting the threshold value $\left(19 \mathrm{~m}^{-1}\right)$ close to the LAC of lipid, see Fig. 4. (ii) The bone-soft tissue boundary pixels were assigned to bone by expanding (dilating) the bone regions by including an extra pixel around the borders. Moreover, bone cavities containing bone marrow were added to the bone regions using hole filling, see Fig. 3. This segmentation was performed at each iteration of DIRA.

\section{Tissue classification}

Air pixels were decomposed to the lipid and water doublet using the 2MD method. Resulting inaccuracies for pure air pixels had little effect on the calculation of forward projections because of the small mass density of air. On the other hand, mass density and material composition of pixels at the air-soft tissue boundary was realistically modeled. Soft tissues were decomposed to the lipid, protein and water (LPW) triplet using the $3 \mathrm{MD}$ method (section II A). Bone tissues were decomposed to the mineral bone and bone marrow doublet using the 2MD method (section IIB). Elemental compositions of the doublet and triplet components are in Supplementary material. The decomposition was done for energies of 50 and $88 \mathrm{keV}$ and the resulting mass fractions were 
stored in the CRV.

\section{E. Uncertainty analysis}

\section{Uncertainty of reconstructed linear attenuation coefficients}

Linear attenuation coefficients $\mu_{1}(x, y)$ and $\mu_{2}(x, y)$ for effective energies $E_{1}$ and $E_{2}$, respectively, were provided by DIRA for each of the four homogeneous circular regions (Fig. 3). Corresponding sample mean, $\bar{\mu}$, standard deviation, $\sigma$, and correlation coefficient, $r\left(\mu_{1}, \mu_{2}\right)$, were calculated using the Matlab functions mean, std and corrcoef, respectively. Error, $\epsilon(\bar{\mu})$, of the sample mean was calculated as $\epsilon(\bar{\mu})=\bar{\mu}-\mu^{\mathrm{t}}$, where $\mu^{\mathrm{t}}$ is the true value calculated from the known elemental composition of the phantom (see Eq. 1). Relative error, $\delta(\bar{\mu})$, of the sample mean was calculated as

$$
\delta(\bar{\mu})=\epsilon(\bar{\mu}) / \mu^{\mathrm{t}}=\left(\bar{\mu}-\mu^{\mathrm{t}}\right) / \mu^{\mathrm{t}},
$$

In the computation of uncertainties in mass fractions (section IIIE 2), the sample standard deviation $\sigma$ was used as the estimate of the standard uncertainty, $u(\mu)$, in the linear attenuation coefficient $\mu$ calculated by DIRA. Corresponding relative standard deviation, $u_{\mathrm{r}}(\mu)$, was calculated as $u_{\mathrm{r}}(\mu)=u(\mu) / \mu$. It should be noted that the uncertainty estimate based on $\sigma$ does not include uncertainties arising from inaccuracies in material data and the summation rule. This should not be a problem in practical situations since the quantum noise and image artifacts (the contribution from $\sigma$ ) dominate the uncertainty. Histograms of $\mu_{1}$ and $\mu_{2}$ were plotted and inspected for each considered configuration.

\section{Uncertainty in estimated mass fractions}

Uncertainty in estimated mass fractions was determined in two ways, here denoted as methods A and B. Method A used the $\mu_{1}$ and $\mu_{2}$ of each pixel in the four circular regions to calculate mass fractions (for each such pixel) from Eqs. (5) and (7). Means, standard deviations, correlation coefficients errors, and relative errors for these mass fractions were calculated similarly as for the attenuation coefficients in section IIIE 1 . Note that the method A resembled the purely statistical, type A evaluation of measurement uncertainty [28]. Method B used the type $\mathrm{B}$ evaluation of uncertainty as derived by GUM [28]. Means and standard deviations for $\mu_{1}$ and $\mu_{2}$ described in section III E 1 were used as input data in the method B (see Supplementary material for details). Uncertainties in mass fractions calculated using the methods $\mathrm{A}$ and $\mathrm{B}$ were compared.

\section{F. Hardware and software}

Simulations were performed on a PC with 82 GB RAM and $2 \times$ Intel Xeon X5650 CPU. Each $2.67 \mathrm{GHz}$ CPU had 6 cores and hyper-threading was turned on. OpenMP version of DIRA 2015a [29] was executed under MATLAB 2014a.

\section{RESULTS}

\section{A. Linear attenuation coefficients}

Reconstructed images at energies $E_{1}=50 \mathrm{keV}$ and $E_{2}=88 \mathrm{keV}$ for the slice B, three noise levels ( $L=0,1,2$, Section IIIB and Supplementary material) and the 8th iteration are shown in Fig. 5. The beam hardening artifact was suppressed in all images. Quality of this suppression was notably better for DIRA than for the filtered backprojection with classical water beam hardening correction, see Fig. 6 , which shows the differences between the phantom image processed with a filter having the

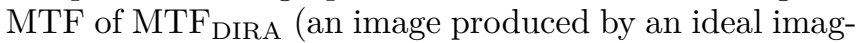
ing system) and corresponding reconstructed images for 0 th and 8th iterations.

Relative errors less than $0.4 \%$ were observed in the four circular regions depicted in Fig. 3, see Table I; the true values were 23.76 and $18.47 \mathrm{~m}^{-1}$ for muscle (region $R_{\mathrm{M}}$ ), 20.19 and $16.59 \mathrm{~m}^{-1}$ for adipose tissue (regions $R_{\mathrm{A}}$ and $R_{\mathrm{C}}$ ), and 28.69 and $20.23 \mathrm{~m}^{-1}$ for femora spongiosa (region $R_{\mathrm{F}}$ ) at energies of 50 and $88 \mathrm{keV}$, respectively.

A small difference between reconstructed and true values was observed for the bones (Fig. 6). Visual inspection of the $50 \mathrm{keV}$ images showed that the reconstructed value had improved from approximately $67.0 \mathrm{~m}^{-1}$ for the 0th iteration to approximately $78.9 \mathrm{~m}^{-1}$ for the 8 th iteration while the true value for bone was $79.3 \mathrm{~m}^{-1}$; the corresponding relative error was reduced from $16 \%$ to $0.5 \%$. The discrepancy between the result for the 8 th iteration and the true value was most likely caused by differences in the implementation of line integral calculations in DIRA and Drasim; the same material cross section data were used in both codes. This discrepancy combined with aliasing is also most likely the cause of dark streaks in no noise images associated with long photon paths through compact bones (Fig. Supp-I for $L=0$.)

Relative standard deviations of the reconstructed linear attenuation coefficients (and consequently the standard deviations of the reconstructed CT numbers) increased with increasing noise level in all four circular regions, see Table I. Note that DIRA did not amplify noise in reconstructed images; the noise was approximately the same for iterations 0 and 8 .

The reconstructed linear attenuation coefficients $\mu_{1}$ and $\mu_{2}$ were correlated (Table I). The correlation was positive for no quantum noise and became negative for noise levels 1 and 2. Most likely, the positive correlation for $L=0$ was caused by weak artifacts resulting 

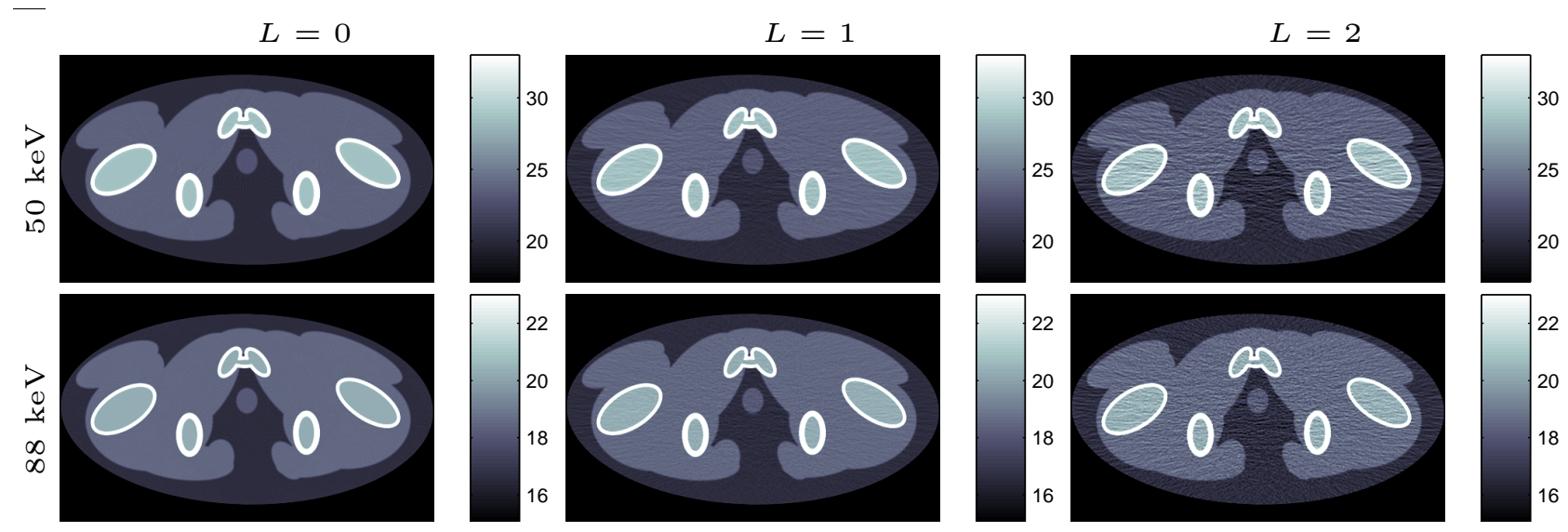

FIG. 5. Reconstructed images at photon energies $E_{1}=50 \mathrm{keV}$ and $E_{2}=88 \mathrm{keV}$ corresponding to tube voltages of 80 and 140 $\mathrm{kV}$ for slice B. Noise levels $L=0,1$, and 2 denote no noise, reduced noise, and a typical noise, respectively (Section III B). Eight iterations of DIRA were used.
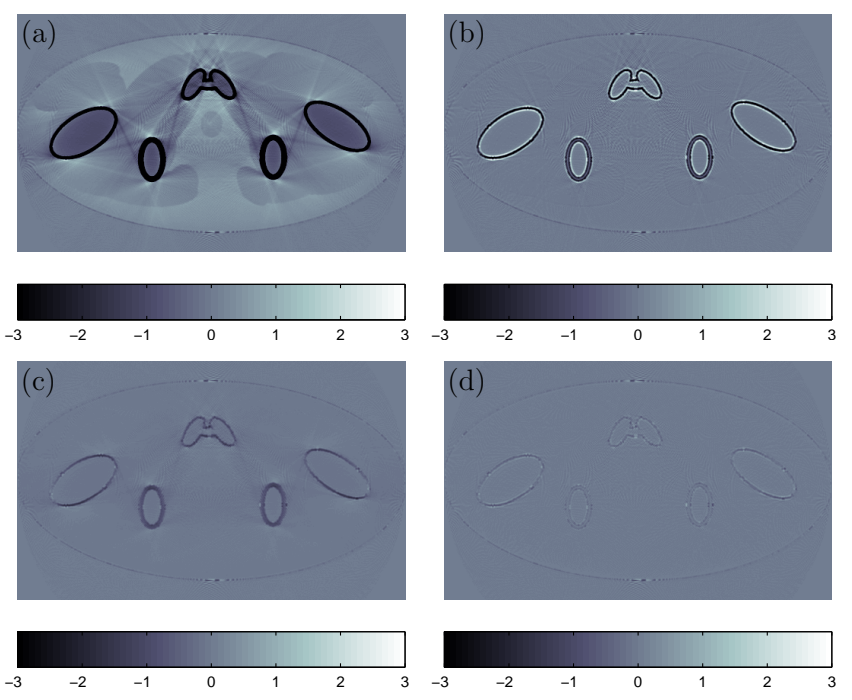

FIG. 6. Difference (in $\mathrm{m}^{-1}$ ) between the reconstructed $\mu$ values at $50 \mathrm{keV}$ and corresponding true $\mu$-values (phantom image) filtered with the system transfer function for 0th (a) and 8th (b) iteration. Similarly for $88 \mathrm{keV}(\mathrm{c}, \mathrm{d})$. The scale is chosen to highlight differences in soft tissues
TABLE I. Relative error $\delta\left(\bar{\mu}_{i}\right)$ of average reconstructed linear attenuation coefficients $\bar{\mu}_{i}$, relative standard uncertainty $u_{\mathrm{r}}\left(\mu_{i}\right)$ of the reconstructed linear attenuation coefficients $\mu_{i}$, standard uncertainty $u\left(H_{i}\right)$ of the corresponding CT number at the effective energy $E_{i}(i=1,2)$ for pixels in four circular regions $R$, three noise levels $L$ and 8 th iteration. The correlation coefficient $r\left(\mu_{1}, \mu_{2}\right)$ is also listed. Values are rescaled using the factor $\%_{0}=10^{-3}$.

\begin{tabular}{rrrrrrrrr}
\hline \hline$R$ & $L$ & $\delta\left(\bar{\mu}_{1}\right)$ & $\delta\left(\bar{\mu}_{2}\right)$ & $u_{\mathrm{r}}\left(\mu_{1}\right)$ & $u_{\mathrm{r}}\left(\mu_{2}\right)$ & $u\left(H_{1}\right)$ & $u\left(H_{2}\right)$ & $r\left(\mu_{1}, \mu_{2}\right)$ \\
& & $(\% 0)$ & $(\% 0)$ & $(\% 0)$ & $(\% 0)$ & & & \\
\hline$R_{\mathrm{M}}$ & 0 & -1.01 & -0.17 & 1.43 & 0.69 & 1 & 1 & 0.94 \\
$R_{\mathrm{M}}$ & 1 & -0.84 & 0.24 & 11.27 & 6.77 & 12 & 7 & -0.30 \\
$R_{\mathrm{M}}$ & 2 & -1.96 & 1.89 & 29.76 & 23.24 & 31 & 24 & -0.18 \\
$R_{\mathrm{A}}$ & 0 & -0.48 & -0.06 & 2.78 & 1.43 & 2 & 1 & 0.99 \\
$R_{\mathrm{A}}$ & 1 & -0.26 & 0.43 & 7.47 & 5.84 & 7 & 5 & -0.06 \\
$R_{\mathrm{A}}$ & 2 & -2.39 & 1.57 & 21.19 & 16.77 & 19 & 16 & -0.20 \\
$R_{\mathrm{C}}$ & 0 & -1.18 & -0.25 & 5.11 & 2.54 & 5 & 2 & 0.99 \\
$R_{\mathrm{C}}$ & 1 & -1.05 & 0.74 & 17.12 & 10.67 & 15 & 10 & -0.04 \\
$R_{\mathrm{C}}$ & 2 & 1.79 & 2.74 & 62.62 & 30.36 & 56 & 28 & 0.01 \\
$R_{\mathrm{F}}$ & 0 & -1.27 & -0.13 & 1.03 & 0.56 & 1 & 1 & 0.96 \\
$R_{\mathrm{F}}$ & 1 & -0.68 & 0.63 & 13.10 & 7.35 & 17 & 8 & -0.22 \\
$R_{\mathrm{F}}$ & 2 & -2.78 & 1.54 & 44.88 & 24.09 & 57 & 28 & -0.22 \\
\hline \hline
\end{tabular}

\section{B. Mass fractions}

from the aliasing, which were present in both $\mu_{1}$ and $\mu_{2}$ images. The overall effect of this correlation was, however, very small since the corresponding covariance was less than $8 \times 10^{-3}$ (see Supplementary material). For $L=1,2$, the noise resulted in close to zero correlation coefficients between $\mu_{1}$ and $\mu_{2}$ for the 0 th iteration. The correlation coefficient was a decreasing function of the number of iteration; the decrease stabilized between 3rd and 5th iteration to the (mostly) negative values of the 8th iteration listed in Table I.
The three-material decomposition of soft tissue to the (lipid, protein, water) triplet resulted in per-pixel mass fractions shown in Fig. 7 for all noise levels $L=$ $0,1,2$ and iteration 8 ; corresponding true values were $(-0.128,0.128,1.000)$ and $(0.701,0.029,0.270)$ for the muscle and adipose tissue, respectively. The negative mass fraction of -0.128 for lipid in the muscle tissue is related to the fact that muscle is outside the (lipid, protein, water) triangle in Fig. 4, see Section V and Ref. 18 for more information. Fig. 7 also demonstrates an im- 
provement in accuracy of the mass fractions achieved by DIRA at iteration 8 compared to the classical water beam hardening correction (iteration 0 ).

The achieved accuracy is quantified in Table II, which lists errors $\epsilon\left(\bar{w}_{1}\right), \epsilon\left(\bar{w}_{2}\right)$, and $\epsilon\left(\bar{w}_{3}\right)$ of average mass fractions of lipid, protein and water, respectively, in the soft tissue regions $R_{\mathrm{M}}, R_{\mathrm{A}}$ and $R_{\mathrm{C}}$ for all noise levels. Also listed is the relative error $\delta(\bar{\rho})$ of average mass density, where the per-pixel mass density was calculated from Eq. (2) and the mass density of the phantom material was used as the true density.

Noise levels in resulting mass fractions and densities were described by standard uncertainties estimated from standard deviations of the per-pixel mass fractions $w_{i}$ and mass density $\rho$, see Table II. As expected, these uncertainties were low for the noise level $L=0$ (no quantum noise), where the variation was caused by small aliasing artifacts only. For the noise level $L=1$ the uncertainty in mass fractions was lower than 0.36. For the noise level $L=2$, however, the standard uncertainties were quite high, e.g. up to 1.2. In this case, individual pixel values were very unreliable and an averaging over a region was needed. The mass fractions and the mass density were highly correlated, for instance $r\left(w_{1}, w_{3}\right) \approx-1$, $r\left(w_{1}, w_{2}\right)>0.93$ and $r\left(w_{2}, \rho\right)>0.94$ for noise levels $L=1,2$. The high correlation was expected since the four output variables $\left(w_{1}, w_{2}, w_{3}, \rho\right)$ were computed from only two input variables $\left(\mu_{1}, \mu_{2}\right)$.

The two-material decomposition of the bone to the (compact bone, bone marrow) doublet was associated with errors, $\epsilon\left(\bar{w}_{1}\right)$ and $\epsilon\left(\bar{w}_{2}\right)$, of average mass fractions and the relative error, $\delta(\bar{\rho})$, of average mass density listed in Table III for the region $R_{\mathrm{F}}$. Corresponding true values were $(0.203,0.797)$ for the mass fractions and $1.123 \mathrm{gcm}^{-3}$ for the mass density. The latter was calculated from Eq. (6), it was not the mass density of the phantom. This choice was motivated by the fact that (i) the relative difference between the two densities was small $(0.07 \%)$, and (ii) the other choice obscured the dependence of the mass density on the noise level. The per-pixel mass fractions $w_{1}$ and $w_{2}$ fulfill the normalization condition. As a consequence, $\bar{w}_{1}+\bar{w}_{2}=1$, $\epsilon\left(\bar{w}_{1}\right)=-\epsilon\left(\bar{w}_{2}\right), u\left(w_{2}\right)=u\left(w_{1}\right)$, and correlation coefficients are $r\left(w_{1}, w_{2}\right)=-1$ and $r\left(w_{1}, \rho\right)=-r\left(w_{2}, \rho\right)$, see Supplementary material.

The convergence speed of DIRA is demonstrated in Fig. 8, which for slice A and noise level $L=2$ shows that the average mass fractions were stable after the 4 th iteration and, in most cases, close to the true values. An exception was the central adipose region $R_{\mathrm{C}}$ for the noise level $L=2$ in slice A for which a small bias was observed; this position was more affected by noise than the other regions, and noise may bias the linear attenuation coefficient [30].

\section{Comparison of method $A$ and method $B$}

Standard uncertainties $u\left(w_{1}\right), u\left(w_{2}\right)$ and $u\left(w_{3}\right)$ in perpixel mass fractions of protein, lipid and water, respectively, determined using the method A (section IIIE2) and presented in Table II for the regions $R_{\mathrm{M}}, R_{\mathrm{A}}$ and $R_{\mathrm{C}}$ were compared to standard uncertainties determined using the method B. Relative differences between results of these two methods were lower than $1.5 \%$ for all cases. Similar comparison was performed for uncertainties in the mass fractions of compact bone and bone marrow for the femora spongiosa region $R_{\mathrm{F}}$; the relative differences were lower than $0.2 \%$. These results indicate that method B can be used for a quick and sufficiently accurate estimate of uncertainties in mass fractions computed using the $2 \mathrm{MD}$ or $3 \mathrm{MD}$ methods.

\section{DISCUSSION}

DIRA belongs to the class of iterative filtered backprojection methods (IFBP) $[12,31]$ whose main advantage is the high convergence speed relative to other iterative methods. Their disadvantage compared to statistical methods such as the iterative maximum-likelihood polychromatic algorithm for CT [9] is worse noise suppression. Methods inspired by the (i) basis decomposition algorithm [11] by Alvarez and Macovski such as MDIR [12] and E-ART [13] or (ii) the RAPCTI algorithm [14] decompose the resulting images into two base materials. Such methods effectively suppress the beam hardening artifact. DIRA extends the material decomposition approach by allowing either the $2 \mathrm{MD}$ or the $3 \mathrm{MD}$ method in the iterative loop. More generally, DIRA provides a framework where prior information about the anatomy [32] can be used for the selection of tissue-specific or contrast media-specific doublets (2MD) and triplets (3MD). The successful use of $3 \mathrm{MD}$ on reconstructed DECT images has been reported $[16,33]$. However, the benefits of using 3MD inside the iterative loop - such as the more accurate estimate of tissue composition-remain to be demonstrated. The drawback of $3 \mathrm{MD}$ is a slightly higher computational load as more forward projections must be computed. The principal component analysis of XCOM data showed that the intrinsic dimensionality of the LAC data (which is of interest in spectral CT) is four for low-Z elements $(Z=1-20)$ when reasonable uncertainties in the data are assumed [34]. DECT has only two degrees of freedom. Nevertheless the assumption about the conservation of molar volumes (equation (2)) allows the determination of mass fractions of three base materials. Radiotherapy treatment planning could benefit from the additional information since, for instance, prostatic tumors contain prostate tissue and calcifications with differing amount of calcium, zinc and other elements $[35,36]$.

Conceptually, DIRA is similar to RAPCTI as both algorithms perform the material decomposition at each iteration and the newly obtained material composition is 

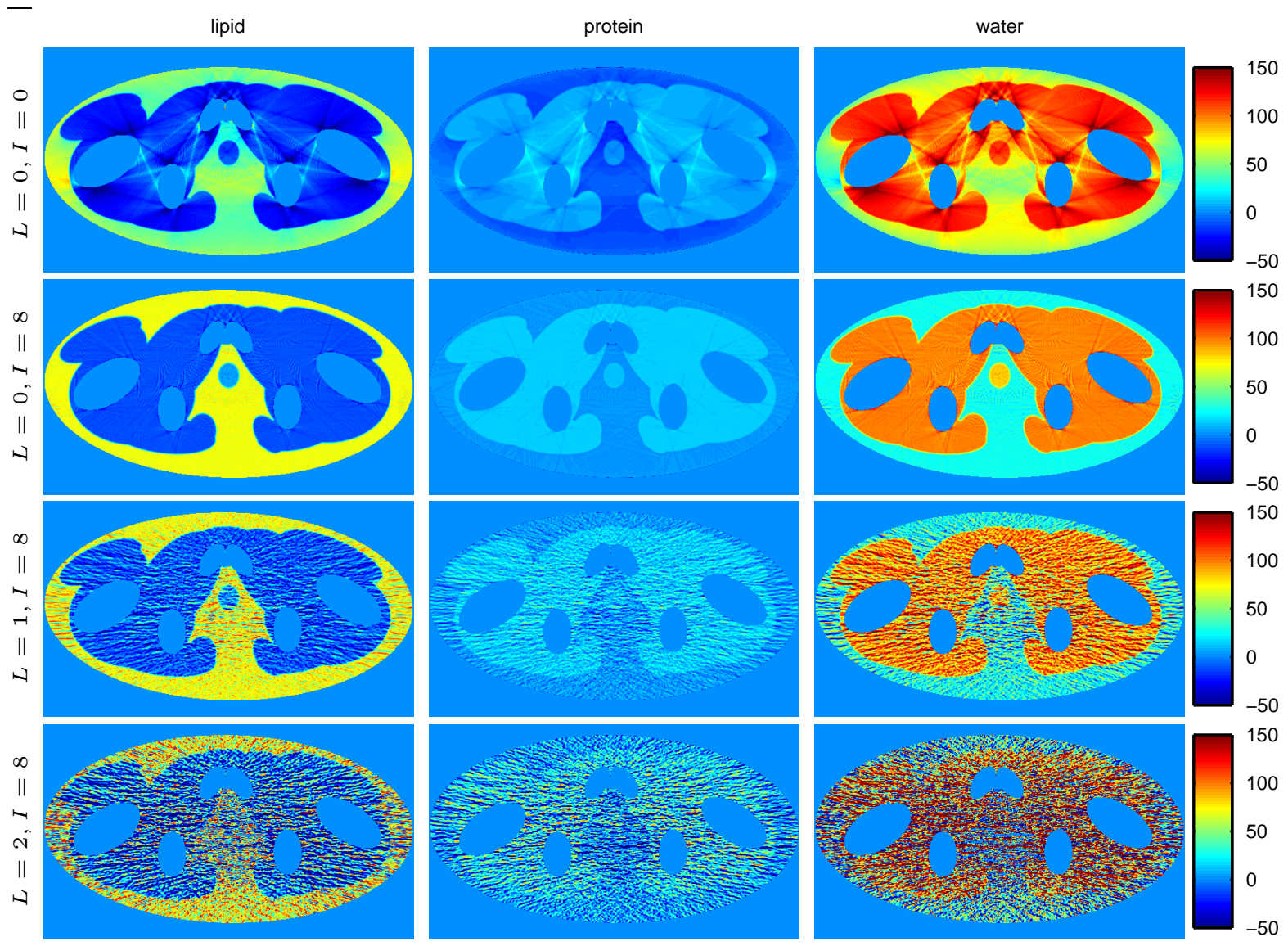

FIG. 7. Mass fractions (in \%) of lipid, protein and water after 0th (first row) and 8th iteration (remaining rows) for noise levels $L=0,1,2$ in slice $\mathrm{B}$.

TABLE II. Errors of average mass fractions of lipid $\epsilon\left(\bar{w}_{1}\right)$, protein $\epsilon\left(\bar{w}_{2}\right)$ and water $\epsilon\left(\bar{w}_{3}\right)$, and the relative error of average mass density of the mixture $\delta(\bar{\rho})$ for the regions $R_{\mathrm{M}}, R_{\mathrm{A}}, R_{\mathrm{C}}$, noise levels $L=0,1,2$ and 8th iteration. Also shown are standard uncertainties $u\left(w_{i}\right)$ and $u(\rho)$ in per-pixel mass fractions and mass density, respectively. All listed quantities were calculated using the method A. Values are rescaled using the factor $\%=0.01$.

\begin{tabular}{rrrrrrrrrr}
\hline \hline$R$ & $L$ & $\begin{array}{r}\epsilon\left(\bar{w}_{1}\right) \\
(\%)\end{array}$ & $\begin{array}{r}\epsilon\left(\bar{w}_{2}\right) \\
(\%)\end{array}$ & $\begin{array}{r}\epsilon\left(\bar{w}_{3}\right) \\
(\%)\end{array}$ & $\begin{array}{r}\delta(\bar{\rho}) \\
(\%)\end{array}$ & $\begin{array}{r}u\left(w_{1}\right) \\
(\%)\end{array}$ & $\begin{array}{r}u\left(w_{2}\right) \\
(\%)\end{array}$ & $\begin{array}{r}u\left(w_{3}\right) \\
(\%)\end{array}$ & $\begin{array}{r}u(\rho) \\
\left(\mathrm{g} \mathrm{cm}^{-3}\right)\end{array}$ \\
\hline$R_{\mathrm{M}}$ & 0 & 1.04 & 0.44 & -1.48 & -0.33 & 1.08 & 0.32 & 1.36 & $0.05 \times 10^{-2}$ \\
$R_{\mathrm{M}}$ & 1 & 1.04 & 0.59 & -1.63 & -0.27 & 17.00 & 10.69 & 27.54 & $1.50 \times 10^{-2}$ \\
$R_{\mathrm{M}}$ & 2 & 2.50 & 1.65 & -4.15 & 0.06 & 46.44 & 30.98 & 76.79 & $4.67 \times 10^{-2}$ \\
$R_{\mathrm{A}}$ & 0 & 0.46 & 0.21 & -0.67 & -0.08 & 1.76 & 0.24 & 1.96 & $0.10 \times 10^{-2}$ \\
$R_{\mathrm{A}}$ & 1 & 0.58 & 0.49 & -1.07 & -0.01 & 9.86 & 7.26 & 16.87 & $1.01 \times 10^{-2}$ \\
$R_{\mathrm{A}}$ & 2 & 3.17 & 2.19 & -5.37 & 0.29 & 29.46 & 21.91 & 50.80 & $3.01 \times 10^{-2}$ \\
$R_{\mathrm{C}}$ & 0 & 1.07 & 0.42 & -1.49 & -0.07 & 3.31 & 0.49 & 3.75 & $0.17 \times 10^{-2}$ \\
$R_{\mathrm{C}}$ & 1 & 1.46 & 1.04 & -2.50 & 0.08 & 21.08 & 14.39 & 34.97 & $1.89 \times 10^{-2}$ \\
$R_{\mathrm{C}}$ & 2 & -2.94 & -0.90 & 3.84 & 0.28 & 73.69 & 46.26 & 118.49 & $5.61 \times 10^{-2}$ \\
\hline \hline
\end{tabular}

used for the computation of the correction to the measured projections. DIRA, however, does not work with $\mathrm{x}$-ray spectrum weighted linear attenuation coefficients; it works with linear attenuation coefficients at the effective energies $E_{1}$ and $E_{2}$. Also, RAPCTI was primarily designed for single-energy CT and uses only one material doublet in DECT.

The main application area of DIRA is QCT for radiotherapy, specifically the determination of elemental composition and mass densities of imaged tissues. This article focuses on the application of the 2MD and 3MD methods in an iterative reconstruction algorithm. Ele- 
TABLE III. Errors of average mass fractions of mineral bone $\epsilon\left(\bar{w}_{1}\right)$ and bone marrow $\epsilon\left(\bar{w}_{2}\right)$, and relative error of average mass density of the mixture $\delta(\bar{\rho})$ for the region $R_{\mathrm{F}}$, noise levels $L=0,1,2$ and 8th iteration. Also shown are corresponding per-pixel standard uncertainties $u\left(w_{1}\right)$ and $u(\rho)$ and the correlation coefficient $r\left(w_{1}, \rho\right)$. Values are rescaled using the factor $\%=0.01$.

\begin{tabular}{|c|c|c|c|c|c|c|}
\hline$L$ & $\begin{array}{r}\epsilon\left(\bar{w}_{1}\right) \\
(\%)\end{array}$ & $\begin{array}{r}\epsilon\left(\bar{w}_{2}\right) \\
(\%)\end{array}$ & $\begin{array}{r}\delta(\bar{\rho}) \\
(\%)\end{array}$ & $\begin{array}{r}u\left(w_{1}\right) \\
(\%)\end{array}$ & $\begin{array}{r}u(\rho) \\
\left(\mathrm{g} \mathrm{cm}^{-3}\right)\end{array}$ & $r\left(w_{1}, \rho\right)$ \\
\hline 0 & -0.183 & 0.183 & 0.01 & 0.08 & $0.05 \times 10^{-2}$ & 0.760 \\
\hline 1 & -0.187 & 0.187 & 0.09 & 2.64 & $1.16 \times 10^{-2}$ & -0.833 \\
\hline 2 & -0.443 & 0.443 & 0.26 & 8.92 & $3.84 \times 10^{-2}$ & -0.828 \\
\hline
\end{tabular}
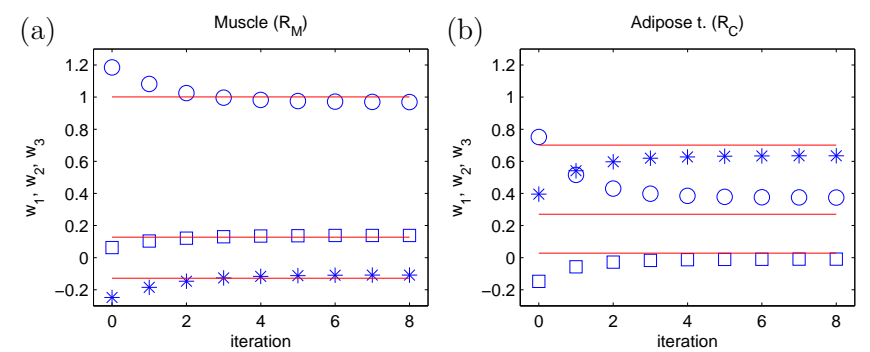

FIG. 8. Average mass fractions of lipid $\bar{w}_{1}(*)$, protein $\bar{w}_{2}$ $(\square)$ and water $\bar{w}_{3}(\circ)$ as functions of the iteration number for muscle in region $R_{\mathrm{M}}$ (a) and adipose tissue in region $R_{\mathrm{C}}$ (b) for noise level $L=2$ and slice A. True values are plotted with horizontal solid lines.

mental mass fractions can be determined from the mass fractions of doublets and triplets using for instance the method described in Ref. 18. The use of several doublets and triplets as in the multimaterial decomposition [33] avoids negative mass fractions. Of interest is the accuracy of these estimates and their applicability in Monte Carlo codes for radiation transport and dose calculations. This topic, including a comparison with other methods for the estimation of elemental composition from DECT scans, is beyond the scope of this article.

A major challenge in the determination of mass fractions is the statistical noise. A moderate noise in projection data may results in bias and large noise in the mass fractions. A workaround is to increase the tube load as in the presented simulations. Though this is typically not a serious problem for patients undergoing a radiotherapy, a better solution is desired. Without regularization (i.e. setting $\mathcal{F}_{H P}\left(\mathbf{f}^{(i)}\right)=0$ in Eq. (12)), experiments not reported here indicated that the noise increased with iterations. This was also concluded in Ref. 31. No regularization was used in Ref. 12. For better noise handling in the future, photon statistic modeling and regularization based on prior object knowledge [8] should be exploited for DIRA.

The number of iterations and stability of the algorithm also affect the accuracy of results. Fig. 8 and figures in Supplementary material show that the algorithm stabi- lized after approximately 4 or 5 iterations. For safety reasons, results were reported for the 8 th iteration. The computation of 1 iteration took approximately $1.3 \mathrm{~s}$ on the 12-core system; the total time of $20 \mathrm{~s}$ for 10 iterations was still acceptable for the clinical practice of radiation treatment planning.

The representation of object materials via doublets and triplets allows to speed up the calculation of forward projections. Ref. 8 claims that x-ray beams can be modeled polychromatic by dividing the spectrum into a number of energy bins and computing each interaction for each bin. It claims that the computational expense would be very high. The implementation in DIRA lowered the time complexity of the algorithm from $O(K N)$ to $O(K+N)$, where $N$ and $K$ are the numbers of voxels and energy bins, respectively, see Supplementary material. In our case $N=512$ and $K=100$.

The classical WBHC algorithm performed at step 0 reduces the number of iterations needed to achieve the same accuracy by about 2, e.g., from 7 to 5, (http://urn.kb.se/resolve?urn=urn:nbn:se:liu:diva80964) compared to a situation when the algorithm is not used. If needed, WBHC can be removed from DIRA or replaced with a different algorithm, e.g., the method by Alvarez and Macovski.

The robustness of the algorithm, i.e., its sensitivity to errors in input quantities like x-ray spectra, has not been systematically investigated in this work. The authors' experience is that the algorithm converges even for input values deviating from true values; the resulting linear attenuation coefficients will be accordingly biased.

In the present form DIRA has limitations: for instance, forward projections are calculated in axial scanning geometry only, scattered radiation is not simulated and an ideal detector array is used. Implementation of a realistic detector response function and scatter contributions to the projections in the iterative loop (Fig. 1) are principally straight-forward. Addition of scatter could be accomplished, e.g., by Monte Carlo simulations [3739]. However, computational efforts will increase considerably. It was beyond the scope of this paper to deal with these problems before a proof-of-concept for the simpler case was verified. Another future development is to introduce helical scanning to overcome the limitation of using axial geometry only.

\section{SHORT SUMMARY AND CONCLUSIONS}

The simulations showed that DIRA was capable of determining material composition of tissues, which is needed in brachytherapy with low energy $(<50 \mathrm{keV})$ photons and proton therapy. Compared to the filtered backprojection with classical water beam hardening correction, the accuracy of resulting mass fractions of base materials was notably improved, for instance from 1.2 to 1.0 for water in the $R_{\mathrm{C}}$ region, see Fig. 8. The algorithm provided quantitative monoenergetic images with beam 
hardening artifacts removed. Its convergence was fast, image sharpness expressed via the modulation transfer function was maintained, and image noise did not increase with the number of iterations. The derived type $B$ evaluation could be used for the determination of uncertainties in mass fractions from uncertainties in linear attenuation coefficients with errors less than $1.5 \%$. Optimization of the forward projection computation algorithm by eliminating summations over all energy bins in the spectrum for each line integral notably reduced the computational expense of the physical modeling part of the algorithm.

\section{SUPPLEMENTARY MATERIAL}

The supplementary material describes the CT scanner geometry, composition of the material doublets and triplets, determination of MTF and filter functions, determination of noise levels and computational complexity of forward projections. It complements Figs. 5, 7, 8 and Tables II and III with figures and tables for every simulated configuration. Also presented are the probability density functions (PDFs) of reconstructed linear attenu- ation coefficients.

\section{ACKNOWLEDGMENTS}

This work was supported by the Swedish Cancer Foundation Grant CAN 2012/764, CAN 2014/691, ALF Grants Region Östergötland LiO-439731, LiO-528791, LiO-602731 and the Medical Faculty at Linköping University. The authors acknowledge contributions by Oscar Grandell (code implementation and testing) and Bror Robin Westin (definition of the anthropomorphic phantom, code generalization and code optimization). This work has been conducted within the Center for Medical Image Science and Visualization (CMIV) at Linköping University, Sweden and we are grateful for using their state of the art computed tomography system.

\section{DISCLOSURE OF CONFLICTS OF INTEREST}

The authors have no relevant conflicts of interest to disclose.
[1] L. Beaulieu, A. Carlsson Tedgren, J.-F. Carrier, S. D. Davis, F. Mourtada, M. J. Rivard, R. M. Thomson, F. Verhaegen, T. A. Wareing, and J. F. Williamson, "Report of the Task Group 186 on model-based dose calculation methods in brachytherapy beyond the TG43 formalism: Current status and recommendations for clinical implementation," Medical Physics 39, 6208-6236 (2012).

[2] R. Nath, "Dosimetry of interstitial brachytherapy sources: Recommendations of the AAPM Radiation Therapy Committee Task Group No. 43," Medical Physics 22, 209 (1995).

[3] G. Landry, B. Reniers, L. Murrer, L. Lutgens, E. Bloemen-Van Gurp, J.-P. Pignol, B. Keller, L. Beaulieu, and F. Verhaegen, "Sensitivity of low energy brachytherapy Monte Carlo dose calculations to uncertainties in human tissue composition," Medical Physics 37, 5188 (2010).

[4] P. Andreo, "Dose to 'water-like' media or dose to tissue in MV photons radiotherapy treatment planning: Still a matter of debate," Physics in Medicine and Biology 60, 309-337 (2015).

[5] H. Paganetti, "Range uncertainties in proton therapy and the role of Monte Carlo simulations," Physics in Medicine and Biology 57, R99-R117 (2012).

[6] G. Landry, K. Parodi, J. E. Wildberger, and F. Verhaegen, "Deriving concentrations of oxygen and carbon in human tissues using single- and dual-energy CT for ion therapy applications," Physics in Medicine and Biology 58, 5029-5048 (2013).

[7] W. van Elmpt, G. Landry, M. Das, and F. Verhaegen, "Dual energy CT in radiotherapy: Current applications and future outlook," Radiotherapy and Oncology (2016), 10.1016/j.radonc.2016.02.026.

[8] M. Beister, D. Kolditz, and W. A. Kalender, "Iterative reconstruction methods in X-ray CT," Physica Medica 28, 94-108 (2012).

[9] B. De Man, J. Nuyts, P. Dupont, G. Marchal, and P. Suetens, "An iterative maximum-likelihood polychromatic algorithm for CT," Medical Imaging, IEEE Transactions on 20, 999-1008 (2001).

[10] M. Magnusson, A. Malusek, A. Muhammad, and G. Alm Carlsson, "Iterative Reconstruction for Quantitative Tissue Decomposition in Dual-Energy CT," in Image Analysis, Vol. 6688, edited by A. Heyden and F. Kahl (Springer Berlin Heidelberg, Berlin, Heidelberg, 2011) pp. 479-488.

[11] R. E. Alvarez and A. Macovski, "Energy-selective reconstructions in X-ray computerised tomography," Physics in Medicine and Biology 21, 733-744 (1976).

[12] C. Maaß, E. Meyer, and M. Kachelrieß, "Exact dual energy material decomposition from inconsistent rays (MDIR)," Medical Physics 38, 691 (2011).

[13] Y. Zhao, X. Zhao, and P. Zhang, "An Extended Algebraic Reconstruction Technique (E-ART) for Dual Spectral CT," IEEE Transactions on Medical Imaging 34, 761-768 (2015).

[14] C. H. Yan, R. T. Whalen, G. S. Beaupre, S. Y. Yen, and S. Napel, "Reconstruction algorithm for polychromatic CT imaging: Application to beam hardening correction," IEEE Transactions on medical imaging 19, 1-11 (2000).

[15] W. Schneider, T. Bortfeld, and W. Schlegel, "Correlation between CT numbers and tissue parameters needed for Monte Carlo simulations of clinical dose distributions," Physics in Medicine and Biology 45, 459-478 (2000).

[16] X. Liu, L. Yu, A. N. Primak, and C. H. McCollough, "Quantitative imaging of element composition and mass 
fraction using dual-energy CT: Three-material decomposition," Medical Physics 36, 1602 (2009).

[17] L. Yu, X. Liu, and C. H. McCollough, "Prereconstruction three-material decomposition in dualenergy CT," (SPIE, 2009) pp. 72583V-72583V-8.

[18] A. Malusek, M. Karlsson, M. Magnusson, and G. Alm Carlsson, "The potential of dual-energy computed tomography for quantitative decomposition of soft tissues to water, protein and lipid in brachytherapy," Physics in Medicine and Biology 58, 771-785 (2013).

[19] M. Bazalova, J.-F. Carrier, L. Beaulieu, and F. Verhaegen, "Dual-energy CT-based material extraction for tissue segmentation in Monte Carlo dose calculations," Physics in Medicine and Biology 53, 2439-2456 (2008).

[20] A. E. Bourque, J.-F. Carrier, and H. Bouchard, "A stoichiometric calibration method for dual energy computed tomography," Physics in Medicine and Biology 59, 20592088 (2014).

[21] G. Landry, M. Gaudreault, W. van Elmpt, J. E. Wildberger, and F. Verhaegen, "Improved dose calculation accuracy for low energy brachytherapy by optimizing dual energy CT imaging protocols for noise reduction using sinogram affirmed iterative reconstruction," Zeitschrift für Medizinische Physik (2015), 10.1016/j.zemedi.2015.09.001.

[22] N. Hünemohr, H. Paganetti, S. Greilich, O. Jäkel, and J. Seco, "Tissue decomposition from dual energy CT data for MC based dose calculation in particle therapy," Medical Physics 41, 061714 (2014).

[23] N. Hünemohr, B. Krauss, C. Tremmel, B. Ackermann, O. Jäkel, and S. Greilich, "Experimental verification of ion stopping power prediction from dual energy CT data in tissue surrogates," Physics in Medicine and Biology 59, 83-96 (2014).

[24] A. C. Kak and M. Slaney, Principles of Computerized Tomographic Imaging (IEEE Press, 1988).

[25] P. M. Joseph, "An improved algorithm for reprojecting rays through pixel images," Medical Imaging, IEEE Transactions on 1, 192-196 (1982).

[26] ICRP, "ICRP Publication 110: Adult reference computational phantoms," Annals of the ICRP 39, 1-166 (2009).

[27] K. Stierstorfer, "DRASIM: A CT-simulation tool," Internal report (Siemens Medical Engineering, 2007).

[28] JCGM, "Evaluation of measurement data -Guide to the expression of uncertainty in measurement," Tech. Rep. JCGM 100:2008 (Joint Committee for Guides in Metrology, 2008).
[29] A. Örtenberg, M. Magnusson, M. Sandborg, G. Alm Carlsson, and A. Malusek, "Parallelisation of the model-based iterative reconstruction algorithm DIRA," Radiation Protection Dosimetry 169, 405-409 (2016).

[30] P. L. Rajbhandary and N. J. Pelc, "Statistical bias in material decomposition in low photon statistics region," in Proc. SPIE 9412, Vol. 9412, edited by C. Hoeschen, D. Kontos, and T. G. Flohr (2015) p. 94124W.

[31] J. Sunnegårdh and P.-E. Danielsson, "Regularized iterative weighted filtered backprojection for helical conebeam CT," Medical Physics 35, 4173 (2008).

[32] M. Kardell, M. Magnusson, M. Sandborg, G. Alm Carlsson, J. Jeuthe, and A. Malusek, "Automatic segmentation of pelvis for brachytherapy of prostate," Radiation Protection Dosimetry 169, 398-404 (2016).

[33] P. R. S. Mendonca, P. Lamb, and D. V. Sahani, "A Flexible Method for Multi-Material Decomposition of Dual-Energy CT Images," IEEE Transactions on Medical Imaging 33, 99-116 (2014).

[34] H. Bornefalk, "XCOM intrinsic dimensionality for low-Z elements at diagnostic energies," Medical Physics 39, 654 (2012).

[35] D. J. Pope, D. L. Cutajar, S. P. George, S. Guatelli, J. A. Bucci, K. E. Enari, S. Miller, R. Siegele, and A. B. Rosenfeld, "The investigation of prostatic calcifications using $\mu$-PIXE analysis and their dosimetric effect in low dose rate brachytherapy treatments using Geant4," Physics in Medicine and Biology 60, 4335-4353 (2015).

[36] N. Miksys, E. Vigneault, A.-G. Martin, L. Beaulieu, and R. M. Thomson, "Large-scale Retrospective Monte Carlo Dosimetric Study for Permanent Implant Prostate Brachytherapy," International Journal of Radiation Oncology*Biology*Physics 97, 606-615 (2017).

[37] S. Jan, D. Benoit, E. Becheva, T. Carlier, F. Cassol, P. Descourt, T. Frisson, L. Grevillot, L. Guigues, L. Maigne, C. Morel, Y. Perrot, N. Rehfeld, D. Sarrut, D. R. Schaart, S. Stute, U. Pietrzyk, D. Visvikis, N. Zahra, and I. Buvat, "GATE V6: A major enhancement of the GATE simulation platform enabling modelling of CT and radiotherapy," Physics in Medicine and Biology 56, 881-901 (2011).

[38] I. Kawrakow, E. Mainegra-Hing, F. Tessier, and B. R. P. Walters, "The EGSnrc C++ class library," Tech. Rep. PIRS-898 (rev A) (NRC, Ottawa, Canada, 2009).

[39] A. Malusek, M. Sandborg, and G. Alm Carlsson, "CTmod-A toolkit for Monte Carlo simulation of projections including scatter in computed tomography," Computer Methods and Programs in Biomedicine 90, 167-178 (2008). 


\title{
Supplementary material to the article: A model-based iterative reconstruction algorithm DIRA using patient-specific tissue classification via DECT for improved quantitative CT in dose planning
}

\author{
A. Malusek* \\ Radiation Physics, Department of Medical and Health Sciences, Linköping University, Linköping, Sweden \\ M. Magnusson ${ }^{\dagger}$ \\ Computer Vision Laboratory, Department of Electrical Engineering, Linköping University, Linköping, Sweden \\ M. Sandborg $\ddagger$ \\ Radiation Physics, Department of Medical and Health Sciences, Linköping University, Linköping, Sweden \\ G. Alm Carlsson $\ddagger$ \\ Radiation Physics, Department of Medical and Health Sciences, Linköping University, Linköping, Sweden
} (Dated: October 27, 2017)

\footnotetext{
* Alexandr.Malusek@liu.se; Center for Medical Image Science and Visualization (CMIV), Linköping University, Linköping, Sweden

$\dagger$ Radiation Physics, Department of Medical and Health Sciences, Linköping University, Linköping, Sweden; Center for Medical Image Science and Visualization (CMIV), Linköping University, Linköping, Sweden

$\ddagger$ Center for Medical Image Science and Visualization (CMIV), Linköping University, Linköping, Sweden
} 


\section{METHODS}

\section{A. Simulation of measured projections}

Polyenergetic CT projections were calculated using the Drasim simulation code for a configuration resembling a typical DECT scanner; simulation parameters are described below.

$X$-ray spectra: The X-ray spectra were produced by Drasim for tube voltages of 80 and $140 \mathrm{kV}$. For $140 \mathrm{kV}$, an extra $0.4 \mathrm{~mm}$ of $\mathrm{Sn}$ was added. Zero focal spot size was used, bowtie filter was not simulated. Effective energies of these spectra were 50 and $88 \mathrm{keV}$ (section A III B).

Gantry: The number of projections was 1152 for a full rotation of $360^{\circ}$. The source-to-origin distance was $d_{\mathrm{SO}}=$ $595 \mathrm{~mm}$ and the detector-to-origin distance was $d_{\mathrm{DO}}=500 \mathrm{~mm}$. An ideal detector measuring energy fluence was simulated. The full fan beam angle was approximately $50^{\circ}$ and the number of detector elements (channels) was 736 . Each detector element registered 10 sub-rays equidistantly distributed in the fan-direction. The quarter off-set [1, p. 43] was used. The detector element width in z-direction was $d_{\mathrm{z}}=1.8403 \mathrm{~mm}$. It corresponded to the beam width of $d_{\mathrm{z}} \cdot d_{\mathrm{SO}} /\left(d_{\mathrm{SO}}+d_{\mathrm{DO}}\right)=1.8403 \cdot 595 /(595+500)=1 \mathrm{~mm}$ at the iso-center for an axial scan and to the slice thickness of $2 \mathrm{~mm}$ for a helical scan fully and without overlapping covering the imaged object.

Rebinning: Using cubic interpolation, the 3D projections were rebinned to 720 parallel projections in $2 \mathrm{D}$ covering a projection interval of $180^{\circ}$. The number of detector elements was 511 and the detector element size in the $2 \mathrm{D}$ geometry was $0.69 \mathrm{~mm}$. This size also became the pixel size in the reconstructed images.

\section{B. Composition of selected materials}

Elemental compositions of the material doublet (mineral bone, bone marrow) and triplet (lipid, protein, water) used in this work are listed in Table I. Also shown is the material composition of prostate.

TABLE I. Elemental atomic compositions and mass densities, $\rho$, of doublet and triplet base materials used in this work.

\begin{tabular}{|c|c|c|}
\hline Material & $\begin{array}{l}\text { Elemental mass fraction } \\
(\%)\end{array}$ & $\begin{array}{r}\rho \\
\left(\mathrm{g} \mathrm{cm}^{-3}\right) \\
\end{array}$ \\
\hline bone marrow $^{\mathrm{a}}$ & $\begin{array}{l}\text { H } 11.0, \mathrm{C} 52.9, \mathrm{~N} 2.05, \mathrm{O} 33.5, \mathrm{Na} 0.05, \mathrm{P} 0.05, \mathrm{~S} 0.15, \mathrm{Cl} 0.15, \mathrm{~K} 0.1 \text {, } \\
\text { Fe } 0.05\end{array}$ & 1.005 \\
\hline mineral bone ${ }^{\mathrm{b}}$ & $\mathrm{H} 3.6, \mathrm{C} 15.9, \mathrm{~N} 4.2, \mathrm{O} 44.8, \mathrm{Na} 0.3, \mathrm{Mg} 0.2, \mathrm{P} 9.4, \mathrm{~S} 0.3$, Ca 21.3 & 1.920 \\
\hline $\operatorname{lipid}^{\mathrm{c}}$ & H 11.8, C 77.3, O 10.9 & 0.920 \\
\hline $\operatorname{protein}^{\mathrm{c}}$ & H $6.6, \mathrm{C} 53.4, \mathrm{~N} 17.0, \mathrm{O} 22.0, \mathrm{~S} 1.0$ & 1.350 \\
\hline water & H $11.2, \mathrm{O} 88.8$ & 1.000 \\
\hline prostate $^{\mathrm{b}}$ & $\mathrm{H} 10.4, \mathrm{C} 23.1, \mathrm{~N} 2.8, \mathrm{O} 62.7, \mathrm{Na} 0.1, \mathrm{P} 0.2, \mathrm{~S} 0.3, \mathrm{Cl} 0.2, \mathrm{~K} 0.2$ & 1.030 \\
\hline
\end{tabular}

${ }^{a}$ Calculated as a mixture of $50 \%$ red marrow and $50 \%$ yellow marrow (by mass) taken from Ref. 2 .

b From Ref. 3.

c From Ref. 4.

\section{DETERMINATION OF MTF AND FILTER FUNCTIONS}

The modulation transfer function (MTF) describes the spatial frequency response of an imaging system. An MTF curve, e.g., a typical Siemens body kernel [5], starts at 1 at the spatial frequency $0 \mathrm{~cm}^{-1}$, is followed by a small overshoot before declining down to 0 at $8 \mathrm{~cm}^{-1}$. The overshoot close to zero frequency results in over- and undershoots close to edges in the image, which might improve the visibility of organs but are not desired for quantitative measurements. The bandwidth of the MTF cannot exceed the bandwidth of the image when iterative reconstruction is used. In our case, the size of the phantom was $35.2 \mathrm{~cm}$ in the lateral direction and $511 \times 511$ pixels were used. The pixel size of $35.2 / 511=0.069 \mathrm{~cm}$ corresponds to the Nyquist frequency (bandwidth) of $f_{\mathrm{n}}=0.5 / 0.069=7.2464 \mathrm{~cm}^{-1}$. To mimic a typical body kernel while avoiding the problems with over- and undershoots close to edges and keeping 
the MTF bandwidth limited by the Nyquist frequency $f_{\mathrm{n}}$ a simple function of the form $\cos ^{n}()$ was used:

$$
\operatorname{MTF}_{\text {DIRA }}(f)=\left\{\begin{array}{rr}
\cos ^{2.1}\left(\frac{\pi}{2} \cdot \frac{f}{f_{\mathrm{n}}}\right), & f<f_{\mathrm{n}}, \\
0, & \text { otherwise },
\end{array}\right.
$$

The parameter $n=2.1$ provided the best visual match between the MTF and the Siemens' typical body kernel; the maximum difference between the two curves was less than 0.1 .

In simulations presented in this work, the MTF is influenced by: (i) the zero size of the X-ray focus, (ii) the size of the detector elements, (iii) the interpolation used during rebinning from fan-beam to parallel beam, (iv) the interpolation used during backprojection in FBP, (v) the interpolation used during projection generation with Joseph's method and (vi) the smoothing filter function $W(f)$. (In FBP the smoothing filter function is combined with the Ram-Lak filter, which is the ramp filter cut off at the bandwidth frequency.) We recall that in Drasim we set the focus size to 0 and used 10 subrays per detector-element to simulate the rectangular detector response.

The smoothing filters (windows) $W_{0}, W_{A}$ and $W_{B}$ in DIRA were assumed to have the form $W()=\cos ^{m}()$, where the parameter $m$ was chosen so that the $\operatorname{MTF}(f)$ of the imaging system matched the $\operatorname{MTF}_{\text {DIRA }}(f)$ in Eq. (1) for a suitable mathematical phantom. The parameter $m$ in filter $W_{0}$ was obtained for the 0th iteration and corresponding parameters for filters $W_{A}$ and $W_{B}$ were obtained for the 8th iteration. The MTF of the imaging system was determined as follows. A water-filled cylinder positioned in the iso-center was used as the mathematical phantom. In an axial plane, this phantom can be described as a circular disc $f(x, y)$, where $x$ and $y$ are coordinates in the 2D spatial domain. Its Fourier transform $F(u, v)$, where $u$ and $v$ are coordinates in the Fourier domain, is known in analytic form, see e.g. Ref. 6, p. 323. Polyenergetic projections of the cylindrical phantom were obtained using Drasim, rebinned and used as measured projections in DIRA. Let $g(x, y)$ be the reconstructed image. The transfer function, $H(u, v)$, of the imaging system was then given by the ratio of the Fourier transforms of the reconstructed image, $G(u, v)$, and the mathematical phantom, $F(u, v)$, as $H(u, v)=G(u, v) / F(u, v)$. For a shift-invariant imaging system, the MTF can be calculated as $\operatorname{MTF}(f)=|H(u, v)| / H(0,0)$, where $f=\left(u^{2}+v^{2}\right)^{1 / 2}$ (Ref. 7, p. 311). In our case, $H(0,0)=1$ and $H(u, v)$ was a real and rotational symmetric function due to the symmetry of the CT-system. Thus the MTF could be estimated as $\operatorname{MTF}(f) \approx H(u, 0)$. The best agreement for $W_{0}$ was achieved for $m=1$, resulting in

$$
W_{\text {DIRA }}(f)=\left\{\begin{array}{rr}
\cos ^{1}\left(\frac{\pi}{2} \cdot \frac{f}{f_{\mathrm{n}}}\right), & f<f_{\mathrm{n}}, \\
0, & \text { otherwise. }
\end{array}\right.
$$

The combination of $W_{A}$ and $W_{B}$ should result in $W_{\text {DIRA }}$, i.e., $W_{A} W_{B}=W_{\text {DIRA }}$. This work used filters $W_{A}=1$ and $W_{B}=W_{\text {DIRA }}$, which suppressed the noise better than the filters $W_{A}=W_{\text {DIRA }}$ and $W_{B}=1$. Other combinations were also tested, but none of them provided notably better results.

\section{DETERMINATION OF NOISE LEVELS}

To determine the typical noise in clinical CT images of the male pelvis, an analysis of selected images reconstructed with FBP was performed. The analysis showed that typical standard deviations of CT numbers were approximately

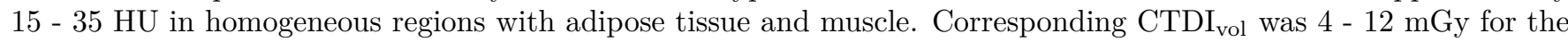
$32 \mathrm{~cm}$ diameter phantom. To avoid comparing noise in such images to noise in images reconstructed by an iterative reconstruction algorithm that automatically reduces noise, only the 0th iteration was used in DIRA. It was found that the per-projection tube loads of 0.217 and $0.08 \mathrm{mAs}$ for the low and high tube voltages, respectively, resulted in standard deviations of $16-31 \mathrm{HU}$ for the $R_{\mathrm{M}}$ and $R_{\mathrm{A}}$ regions (see Table A-I), which were similar to the standard deviations in the clinical images. Simulation setups with these and tenfold increased tube loads are referred to as noise levels $L=2$ and $L=1$, respectively, in this work. The setup with no simulated noise is denoted as $L=0$.

\section{UNCERTAINTY OF COMPUTED MASS FRACTIONS}

Uncertainty in the computed mass fractions depends on many factors, for instance the accuracy of the image reconstruction algorithm, accuracy of the tabulated cross sections as functions of energy, the accuracy of the independent atom approximation, and the accuracy of the formula for density of the mixture (Eq. (A2)). In clinical practice, the dominating factor is typically the quantum noise affecting the detector signal. For low photon intensities, the electronic noise may further reduce the signal-to-noise ratio by 30 to $50 \%$ [8, p. 49]. While the former factors are mostly deterministic and can, in principle, be corrected for, the quantum and electronic noise are random in nature 
and typically determine the precision of the calculated mass fractions. In this section, a simple model is presented for the calculation of uncertainties in mass fractions $w_{i}, 1 \leq i \leq 3$, from uncertainties in linear attenuation coefficients $\mu\left(E_{1}\right)$ and $\mu\left(E_{2}\right)$ at effective photon energies $E_{1}$ and $E_{2}$, respectively, calculated by the reconstruction algorithm. For clarity, these linear attenuation coefficients are denoted as $\mu_{1} \equiv \mu\left(E_{1}\right)$ and $\mu_{2} \equiv \mu\left(E_{2}\right)$. Consider the measurement model $w_{i}=f_{i}\left(\mu_{1}, \mu_{2}\right)$, where the model function $f_{i}$ is given by Eq. (A5). The standard uncertainty $u\left(w_{i}\right)$ in $w_{i}$ can be estimated by $[9]$

$$
u^{2}\left(w_{i}\right)=\left(\frac{\partial f_{i}}{\partial \mu_{1}}\right)^{2} u^{2}\left(\mu_{1}\right)+\left(\frac{\partial f_{i}}{\partial \mu_{2}}\right)^{2} u^{2}\left(\mu_{2}\right)+2 \frac{\partial f_{i}}{\partial \mu_{1}} \frac{\partial f_{i}}{\partial \mu_{2}} u\left(\mu_{1}\right) u\left(\mu_{2}\right) r\left(\mu_{1}, \mu_{2}\right),
$$

where $u\left(\mu_{j}\right)$ is the standard uncertainty in $\mu_{j}$ and $r\left(\mu_{1}, \mu_{2}\right)$ is the correlation coefficient between $\mu_{1}$ and $\mu_{2}$. The sensitivity coefficients $\partial f / \partial \mu_{j}=\left(\partial f_{1} / \partial \mu_{j}, \partial f_{2} / \partial \mu_{j}, \partial f_{3} / \partial \mu_{j}\right)^{T}$ can be calculated as

$$
\frac{\partial f}{\partial \mu_{j}}=\frac{\partial\left(A^{-1} b\right)}{\partial \mu_{j}}=\frac{\partial A^{-1}}{\partial \mu_{j}} b=-A^{-1} \frac{\partial A}{\partial \mu_{j}} A^{-1} b
$$

where the matrix $A$ and the vector $b$ have the same meaning as in Eq. (A3), and $A^{-1}$ is the inverse matrix of $A$. The formula

$$
\frac{\partial A^{-1}}{\partial \mu_{j}}=-A^{-1} \frac{\partial A}{\partial \mu_{j}} A^{-1}
$$

used in Eq. (4) allows the calculation of partial derivatives $\partial A^{-1} / \partial \mu_{j}$, which are difficult to express directly, through partial derivatives $\partial A / \partial \mu_{j}$. Eq. (5) can be derived from the relation $I=A^{-1} A$, where $I$ is the identity (unit) matrix, by multiplying the following equation by $A^{-1}$ from the right-hand side:

$$
O=\frac{\partial I}{\partial \mu_{j}}=\frac{\partial\left(A^{-1} A\right)}{\partial \mu_{j}}=\frac{\partial A^{-1}}{\partial \mu_{j}} A+A^{-1} \frac{\partial A}{\partial \mu_{j}} .
$$

In (6), $O$ is the zero matrix.

The partial derivatives $\partial A / \partial \mu_{j}$ can be calculated directly from Eq. (A3) as

$$
\frac{\partial A}{\partial \mu_{1}}=\left(\begin{array}{ccc}
\rho_{1}^{-1} & \rho_{2}^{-1} & \rho_{3}^{-1} \\
0 & 0 & 0 \\
0 & 0 & 0
\end{array}\right), \frac{\partial A}{\partial \mu_{2}}=\left(\begin{array}{ccc}
0 & 0 & 0 \\
\rho_{1}^{-1} & \rho_{2}^{-1} & \rho_{3}^{-1} \\
0 & 0 & 0
\end{array}\right)
$$

For the 2MD method, the uncertainties $u\left(w_{1}\right), u\left(w_{2}\right)$ and $u\left(\rho^{-1}\right)$ can be calculated from Eq. (3) using the functional relation in Eq. (A7). The sensitivity coefficients can be calculated from Eq. (4), where

$$
\frac{\partial A}{\partial \mu_{1}}=\left(\begin{array}{ccc}
0 & 0 & -1 \\
0 & 0 & 0 \\
0 & 0 & 0
\end{array}\right), \frac{\partial A}{\partial \mu_{2}}=\left(\begin{array}{ccc}
0 & 0 & 0 \\
0 & 0 & -1 \\
0 & 0 & 0
\end{array}\right)
$$

The uncertainty $u(\rho)$ can be estimated as $[9] u(\rho)=\rho^{2} u\left(\rho^{-1}\right)$.

\section{COVARIANCE BETWEEN MASS FRACTIONS}

There is an association between mass fractions computed using the three material decomposition. Consider that the linear attenuation coefficient $\mu_{1}$ measured with the CT scanner at the effective photon energy $E_{1}$ has a distribution with the expectation $E\left(\mu_{1}\right)$ and variance $\mathrm{V}\left(\mu_{1}\right)$. Corresponding quantities for the linear attenuation coefficient $\mu_{2}$ measured at $E_{2}$ are $E\left(\mu_{2}\right)$ and $\mathrm{V}\left(\mu_{2}\right)$.

The covariance between the mass fractions $w_{i}$ and $w_{j}$, which are now considered as functions of random variables $\mu_{1}$ and $\mu_{2}$, can be estimated using the same approach as in Ref. 9. The functional relation $w_{i}=f_{i}\left(\mu_{1}, \mu_{2}\right)$ given by Eq. (A5) is linearized using the Taylor series. The approximate mass fraction $\hat{w}_{i}$ is then

$$
\hat{w}_{i}=f_{i}\left(E\left(\mu_{1}\right), E\left(\mu_{2}\right)\right)+\frac{\partial f_{i}}{\partial \mu_{1}}\left(\mu_{1}-E\left(\mu_{1}\right)\right)+\frac{\partial f_{i}}{\partial \mu_{2}}\left(\mu_{2}-E\left(\mu_{2}\right)\right),
$$


where the partial derivatives are evaluated at the point $\left(E\left(\mu_{1}\right), E\left(\mu_{2}\right)\right)$. The covariance can be calculated from the definition

$$
\mathrm{C}\left(\hat{w}_{i}, \hat{w}_{j}\right) \equiv E\left[\left(\hat{w}_{i}-E\left(\hat{w}_{i}\right)\right)\left(\hat{w}_{j}-E\left(\hat{w}_{j}\right)\right)\right]
$$

by substituting $\hat{w}_{i}$ in Eq. (9) to (10) and realizing that $E\left(\hat{w}_{i}\right)=f_{i}\left(E\left(\mu_{1}\right), E\left(\mu_{2}\right)\right)$ and $\mathrm{C}\left(\mu_{2}, \mu_{1}\right)=\mathrm{C}\left(\mu_{1}, \mu_{2}\right)$ :

$$
\mathrm{C}\left(\hat{w}_{i}, \hat{w}_{j}\right)=\frac{\partial f_{i}}{\partial \mu_{1}} \frac{\partial f_{j}}{\partial \mu_{1}} \mathrm{~V}\left(\mu_{1}\right)+\frac{\partial f_{i}}{\partial \mu_{2}} \frac{\partial f_{j}}{\partial \mu_{2}} \mathrm{~V}\left(\mu_{2}\right)+\left(\frac{\partial f_{i}}{\partial \mu_{1}} \frac{\partial f_{j}}{\partial \mu_{2}}+\frac{\partial f_{i}}{\partial \mu_{2}} \frac{\partial f_{j}}{\partial \mu_{1}}\right) \mathrm{C}\left(\mu_{1}, \mu_{2}\right)
$$

Note that Eq. (3) follows from Eq. (11) since $u^{2}\left(w_{i}\right)=\mathrm{V}\left(w_{i}\right)=\mathrm{C}\left(w_{i}, w_{i}\right)$ for $1 \leq i \leq 3$ and $u^{2}\left(\mu_{j}\right)=\mathrm{V}\left(\mu_{j}\right)$ for $1 \leq j \leq 2$.

For the $2 \mathrm{MD}$, the normalization condition $w_{1}+w_{2}=1$ associates the mass fractions $w_{1}$ and $w_{2}$ so that $\mathrm{C}\left(w_{1}, w_{2}\right)=$ $\mathrm{C}\left(w_{1}, 1-w_{1}\right)=-\mathrm{C}\left(w_{1}, w_{1}\right)=-\mathrm{V}\left(w_{1}\right)=-\mathrm{V}\left(w_{2}\right)$. The correlation coefficient is then

$$
r\left(w_{1}, w_{2}\right) \equiv \frac{\mathrm{C}\left(w_{1}, w_{2}\right)}{\left[\mathrm{V}\left(w_{1}\right) \mathrm{V}\left(w_{2}\right)\right]^{1 / 2}}=-1
$$

and $r\left(w_{2}, \rho\right)=r\left(1-w_{1}, \rho\right)=-r\left(w_{1}, \rho\right)$.

\section{TIME COMPLEXITY OF FORWARD PROJECTIONS}

Time complexity of an algorithm can be quantified by the number of performed elementary operations. Of special interest is the asymptotic behavior of algorithms, when certain parameters approach infinity. In this case the time complexity is commonly described by the big-O (ordo) notation. For instance the time complexity of the order $N$, denoted as $O(N)$, means that the number of elementary operations is proportional to the parameter $N$.

Suppose that a slice of the voxel array has the size of $N \times N$ (e.g. $512 \times 512$ ) and the number of energy bins in the x-ray spectrum is $K$ (e.g. 100). The number of operations performed in a discretized version of Eq. (A18) (see Eq. (A20)) is proportional to $K$ assuming that the integrals $l_{i}$ have been pre-calculated. Since their calculation requires a number of operations that is proportional to $N$, the total time complexity is $O(K+N)$.

On the other hand, the number of operations performed in a discretized version of the expression $\exp [\ldots]$ in Eq. (A16) is proportional to $K N$, thus the complexity is $O(K N)$. The number of operations performed in the discretized remaining part of the formula is proportional to $K$. The total time complexity is thus $O(K+K N)$, which is the same as $O(K N)$.

\section{RESULTS}

Figs. 1 and 2 show reconstructed linear attenuation coefficients at 50 and $88 \mathrm{keV}$, respectively for the 0th (classical water beam hardening correction) and 8th iteration of DIRA. They complement Fig. A-5 in the article.

Figs. 4-9 show normalized probability density functions (PDFs) of reconstructed linear attenuation coefficients in muscle, adipose tissue, femora spongiosa and pelvis spongiosa regions for all pixels (except those close to the edges) and iterations 0 and 8; the regions were defined by manually created masks shown in Fig. 3. The PDFs show that DIRA reduced the bias while the statistical noise (the width of the peaks for noise levels $L=1,2$ ) remained approximately the same.

Figs. 11-13 show calculated mass fractions for slices A-C; they complement Fig. A-7 in the article. The soft tissue was separated by automatic segmentation in the iterative loop of DIRA; Fig. 10 shows masks of all segmented tissues.

Figs. 15-17 demonstrate the convergence speed of DIRA by showing average mass fractions in the four circular regions (Fig. 14) as functions of the number of iterations. They complement Fig. A-8 in the article.

Table II complements Table A-I in the article; it contains information on all three slices A, B and C. It also lists the covariance between linear attenuation coefficients $\mu_{1}$ and $\mu_{2}$. Tables III and IV complement Table A-II, and Tables VI and VII complement Table A-III; they contain information on all three slices. Table V shows that calculated mass fractions and mass densities were highly correlated. 


\section{REFERENCES}

[1] W. Kalender, Computed tomography: fundamentals, system technology, image quality, applications (Publicis Corporate Pub., Erlangen, 2011).

[2] ICRU, Tissue substitutes in radiation dosimetry and measurement., ICRU report 44 (International Commission on Radiation Units and Measurements, Bethesda, Md., U.S.A., 1989).

[3] ICRP, Annals of the ICRP 39, 1 (2009).

[4] ICRU, Photon, electron, proton, and neutron interaction data for body tissues., ICRU report 46 (International Commission on Radiation Units and Measurements, Bethesda, Md., U.S.A., 1992).

[5] Siemens, SOMATOM Definition Flash system owner manual, Tech. Rep. (2011).

[6] L. Råde and B. Westergren, Mathematics handbook for science and engineering (Springer ; Studentlitteratur, Berlin; Lund, 2004).

[7] H. H. Barrett and K. J. Myers, Foundations of Image Science (Wiley-Interscience, Hoboken, NJ, 2004).

[8] B. J. Heismann, B. T. Schmidt, and T. Flohr, Spectral computed tomography (SPIE Press, Bellingham, Wash. (1000 20th St. Bellingham WA 98225-6705 USA), 2012).

[9] JCGM, Evaluation of measurement data - Guide to the expression of uncertainty in measurement, Tech. Rep. JCGM 100:2008 (Joint Committee for Guides in Metrology, 2008). 


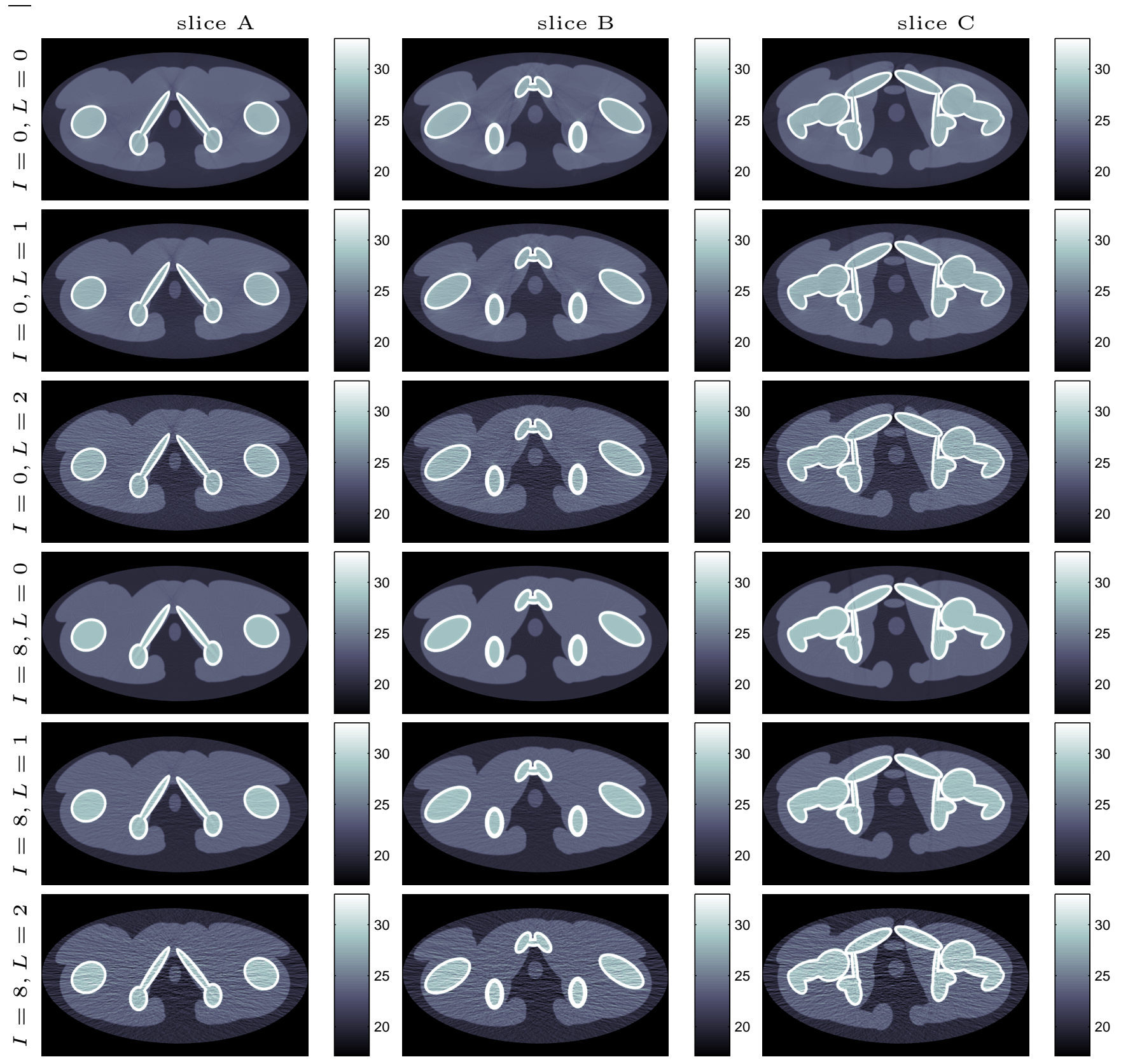

FIG. 1. Reconstructed slices A, B and C for effective photon energy of $E_{1}=50 \mathrm{keV}$ corresponding to the tube voltage of 80 $\mathrm{kV}$, noise levels $L=0,1,2$, and iterations $I=0,8$. 


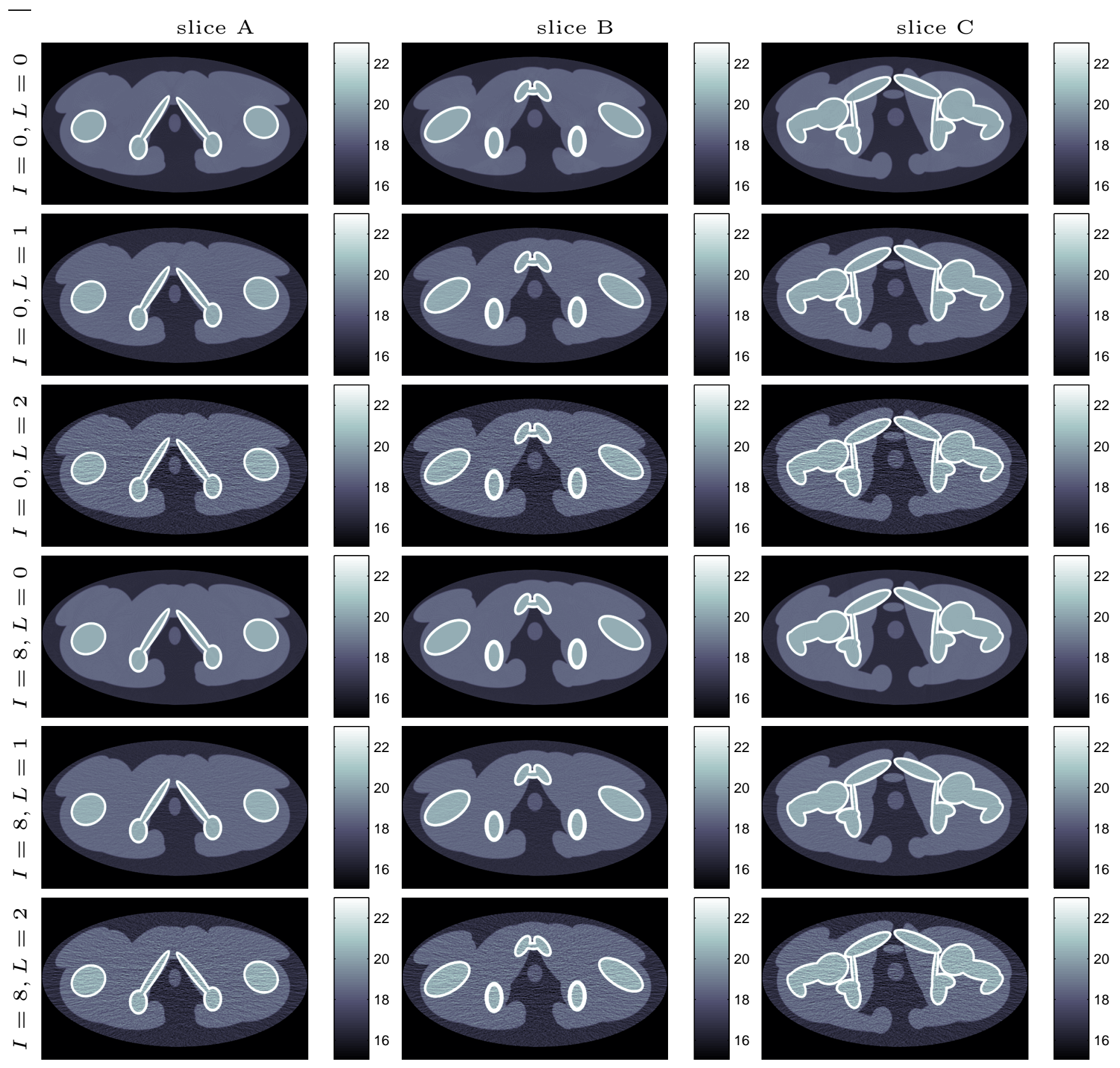

FIG. 2. Same as Fig. 1 for $E_{2}=88 \mathrm{keV}$ corresponding to the tube voltage of $140 \mathrm{kV}$. 
9
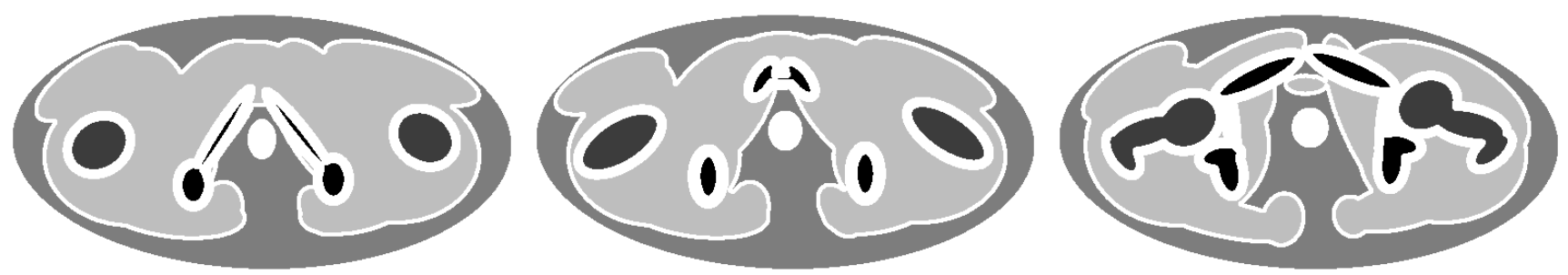

FIG. 3. Manually created masks defining muscle, adipose tissue, femora spongiosa and pelvis spongiosa regions. These masks were used to select pixels for histograms in Figs. 4-9. We recall that these masks were derived from regions defining the phantom by erosion and dilation so that border pixels (plotted as white regions) are not included. 

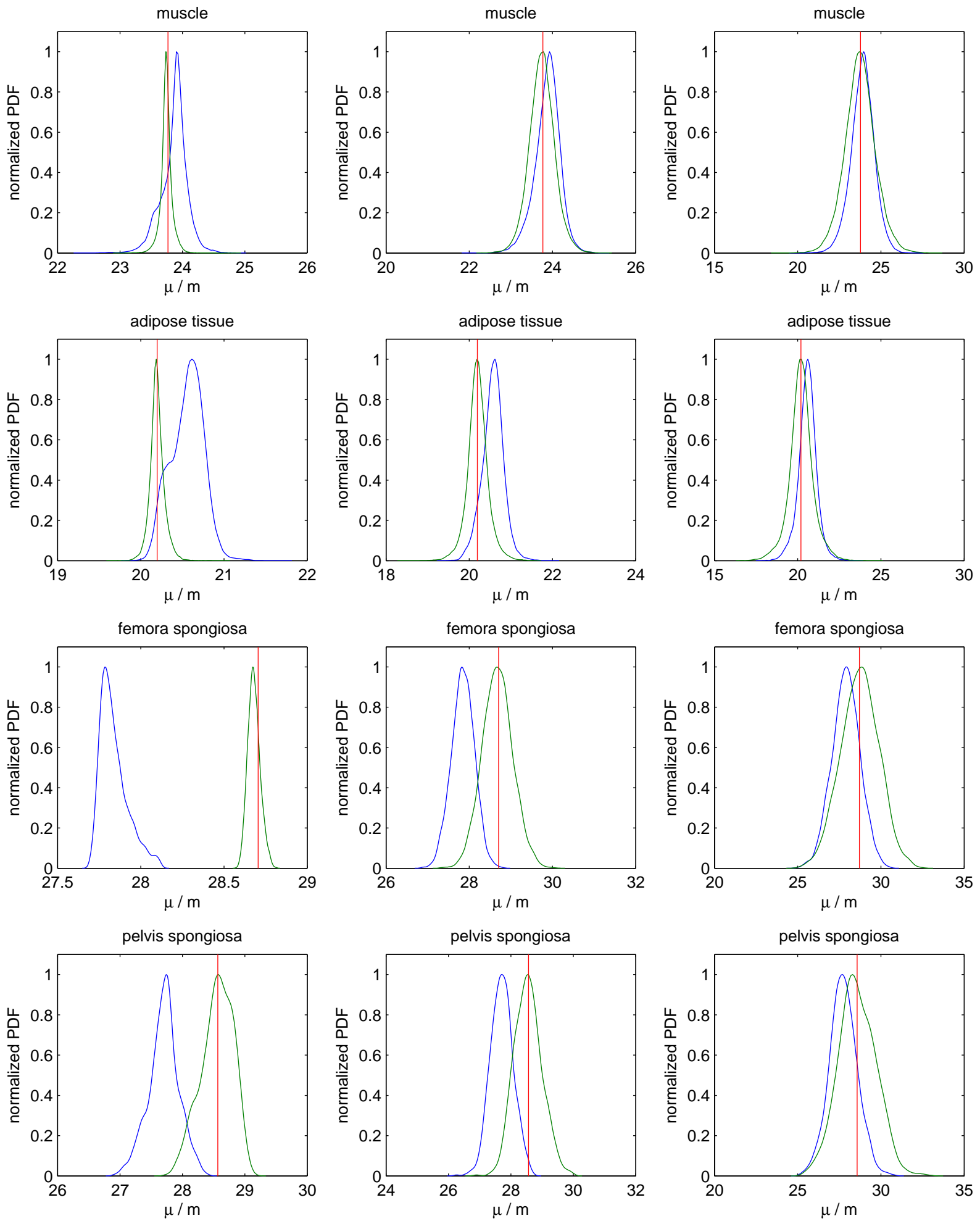

FIG. 4. Normalized probability density function of reconstructed linear attenuation coefficients $\mu$ at $50 \mathrm{keV}$ in the muscle, adipose tissue, femora spongiosa and pelvis spongiosa regions of slice A for iterations 0 (blue) and 8 (green) and noise level $L=0$ (left column), $L=1$ (middle column) and $L=2$ (right column). True values are plotted as a vertical line (red). 

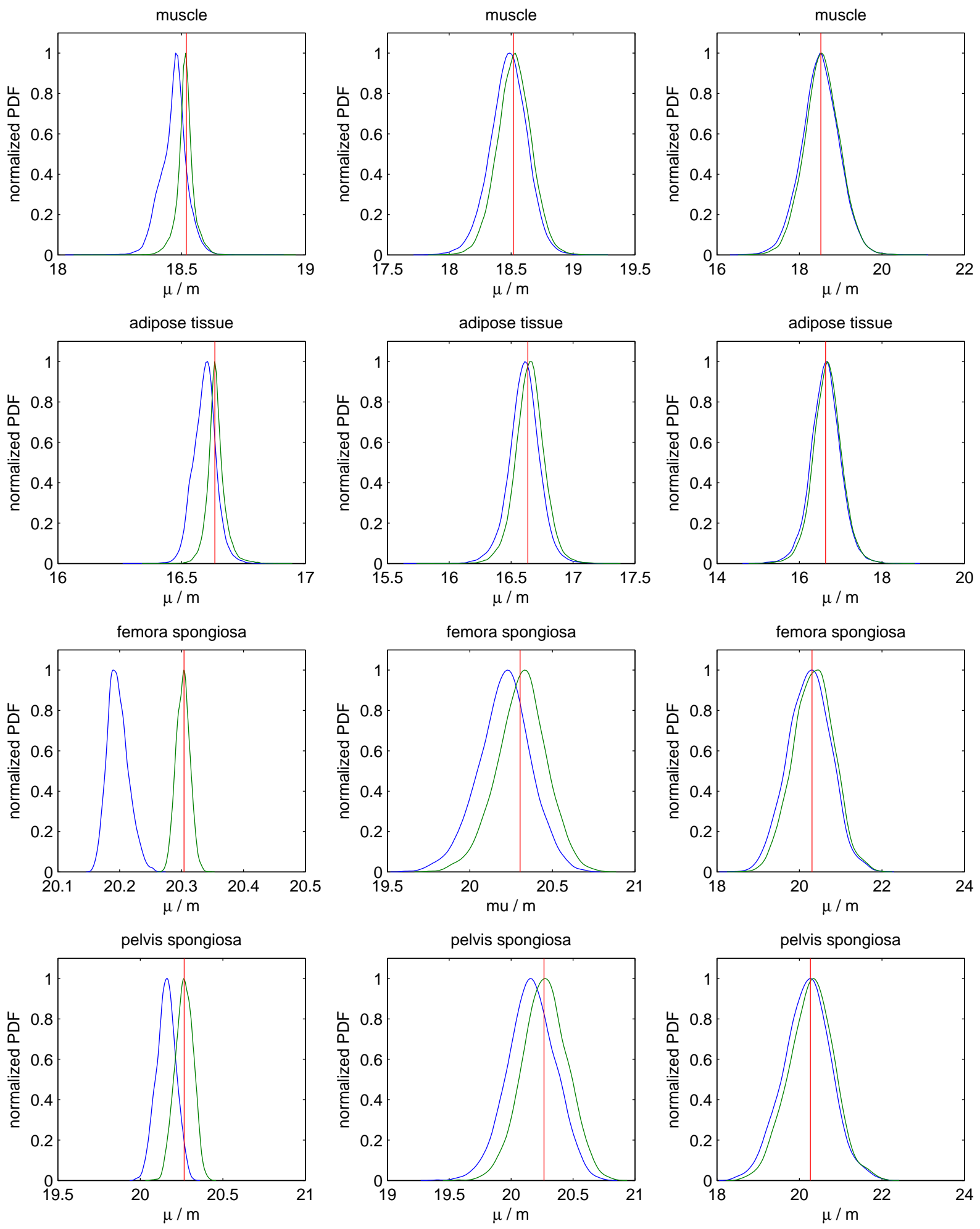

FIG. 5. Same as Fig. 4 for slice $\mathrm{A}$ and $\mu$ at $88 \mathrm{keV}$. 

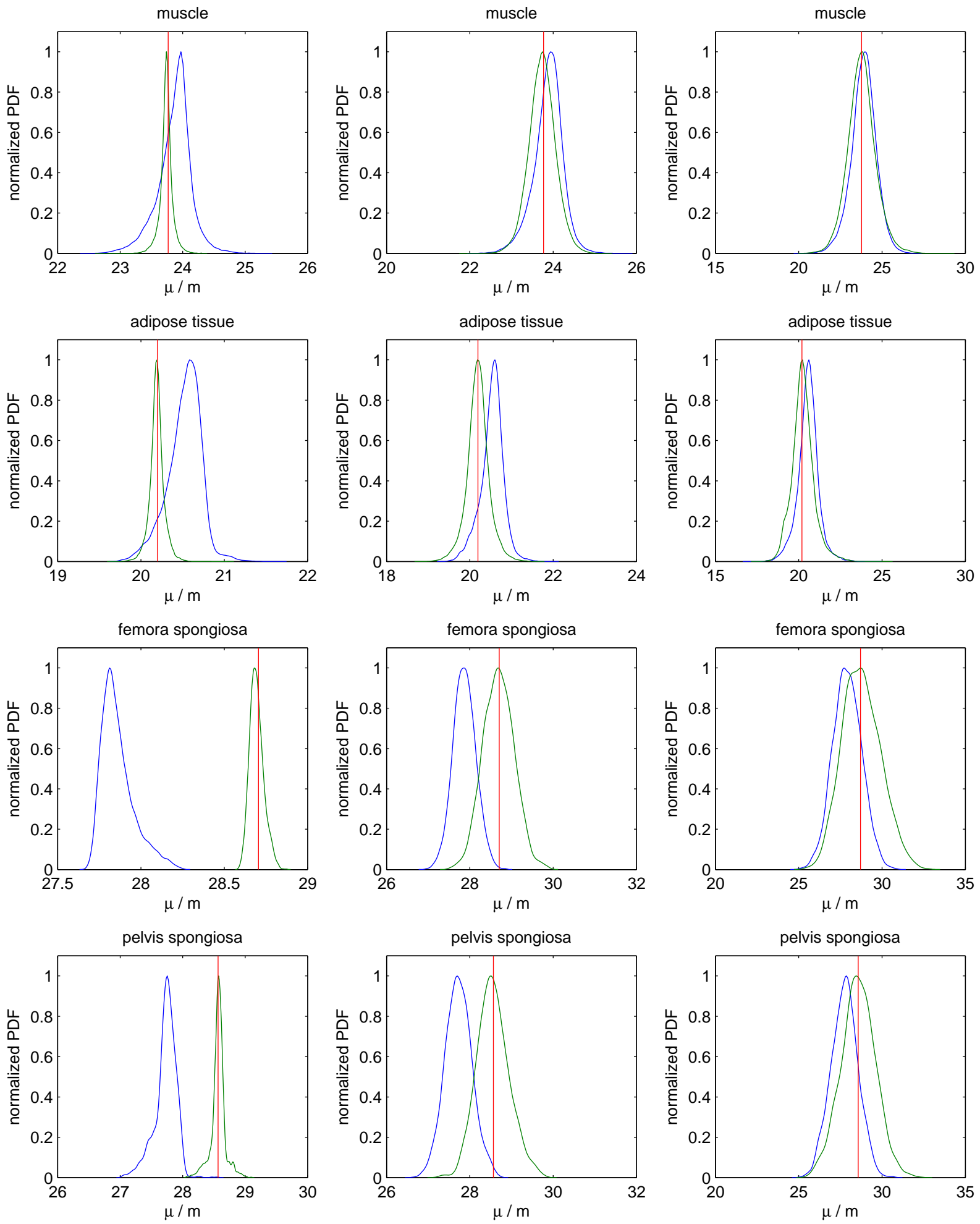

FIG. 6. Same as Fig. 4 for slice B and $\mu$ at $50 \mathrm{keV}$. 

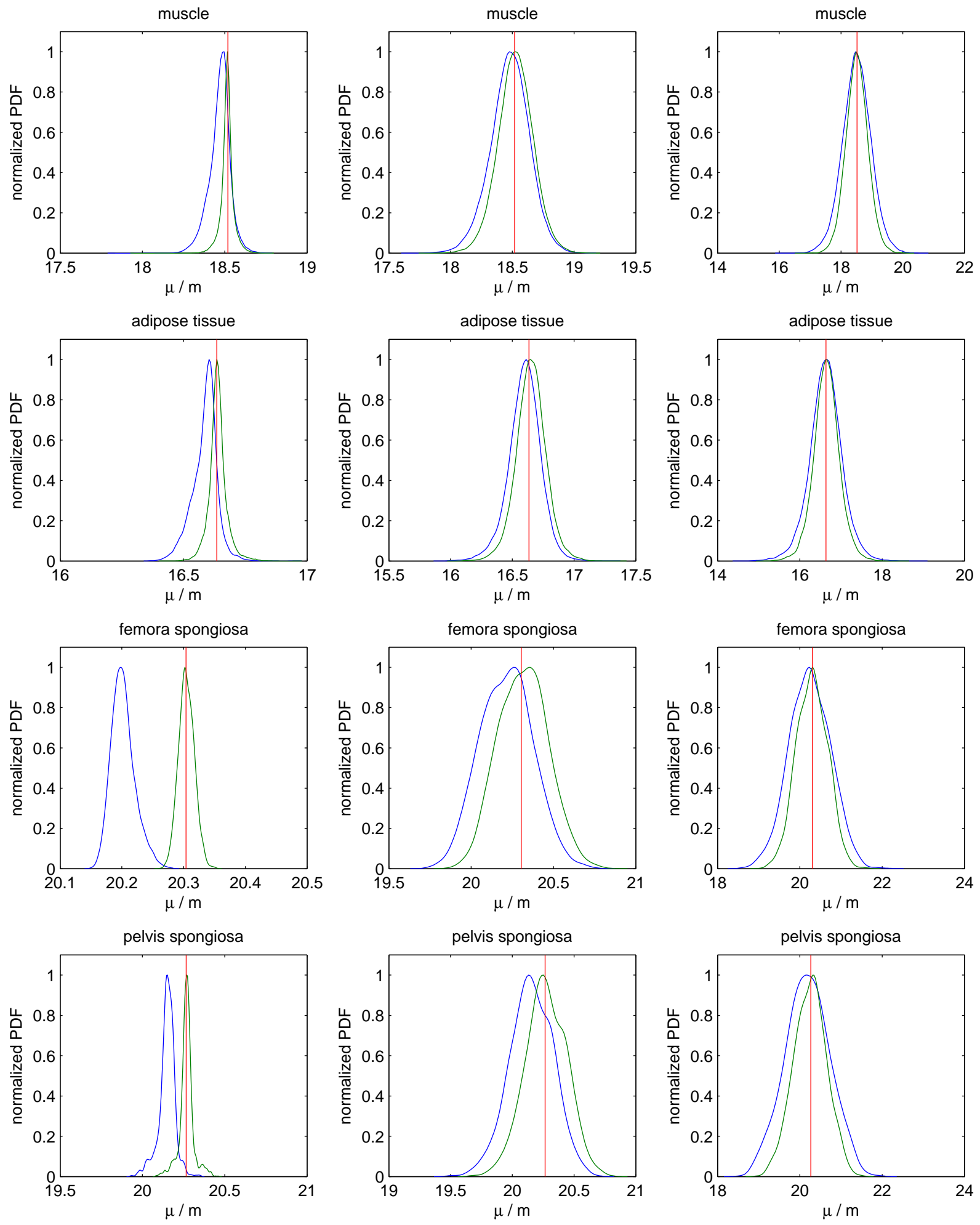

FIG. 7. Same as Fig. 4 for slice B and $\mu$ at $88 \mathrm{keV}$. 

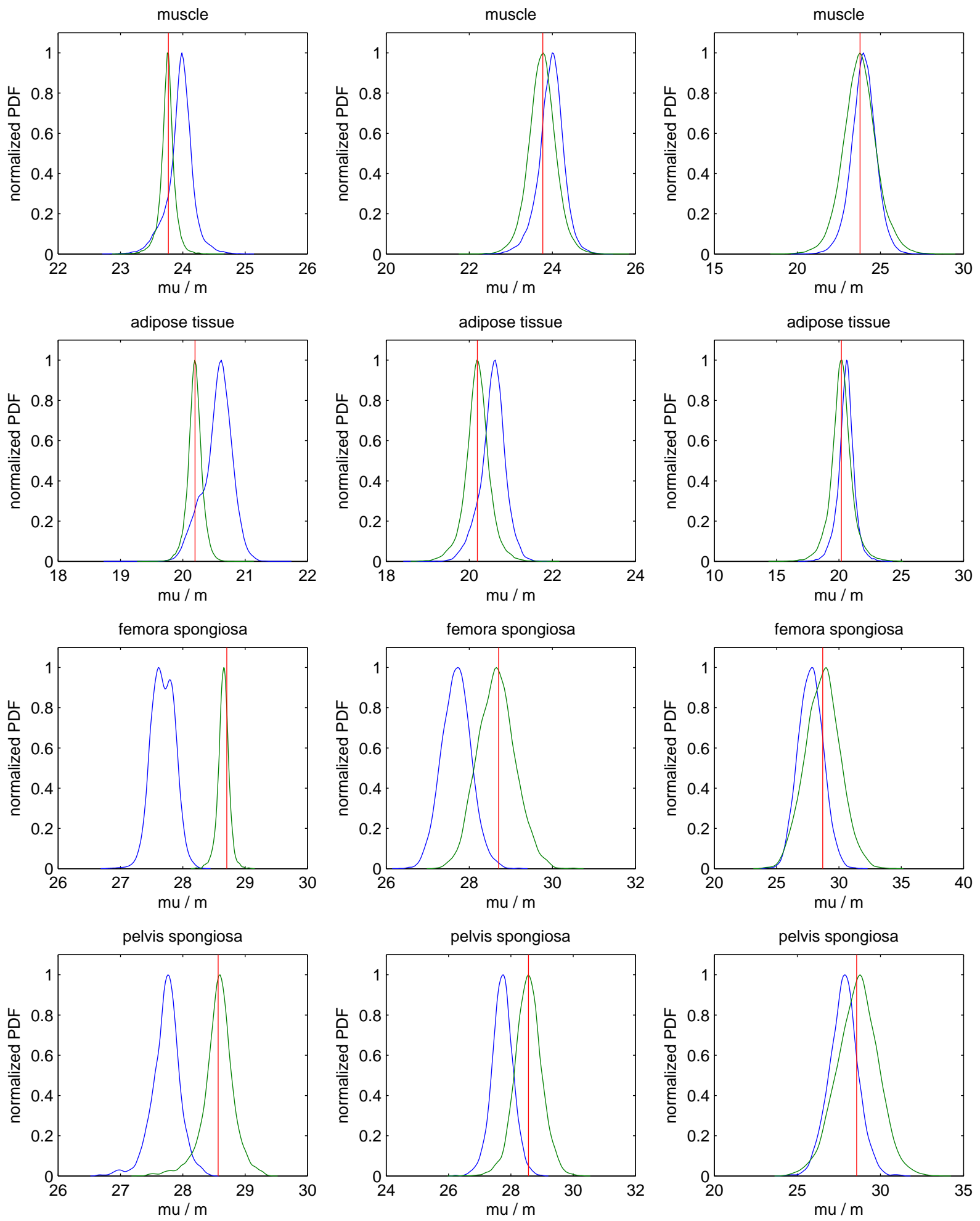

FIG. 8. Same as Fig. 4 for slice $\mathrm{C}$ and $\mu$ at $50 \mathrm{keV}$. 

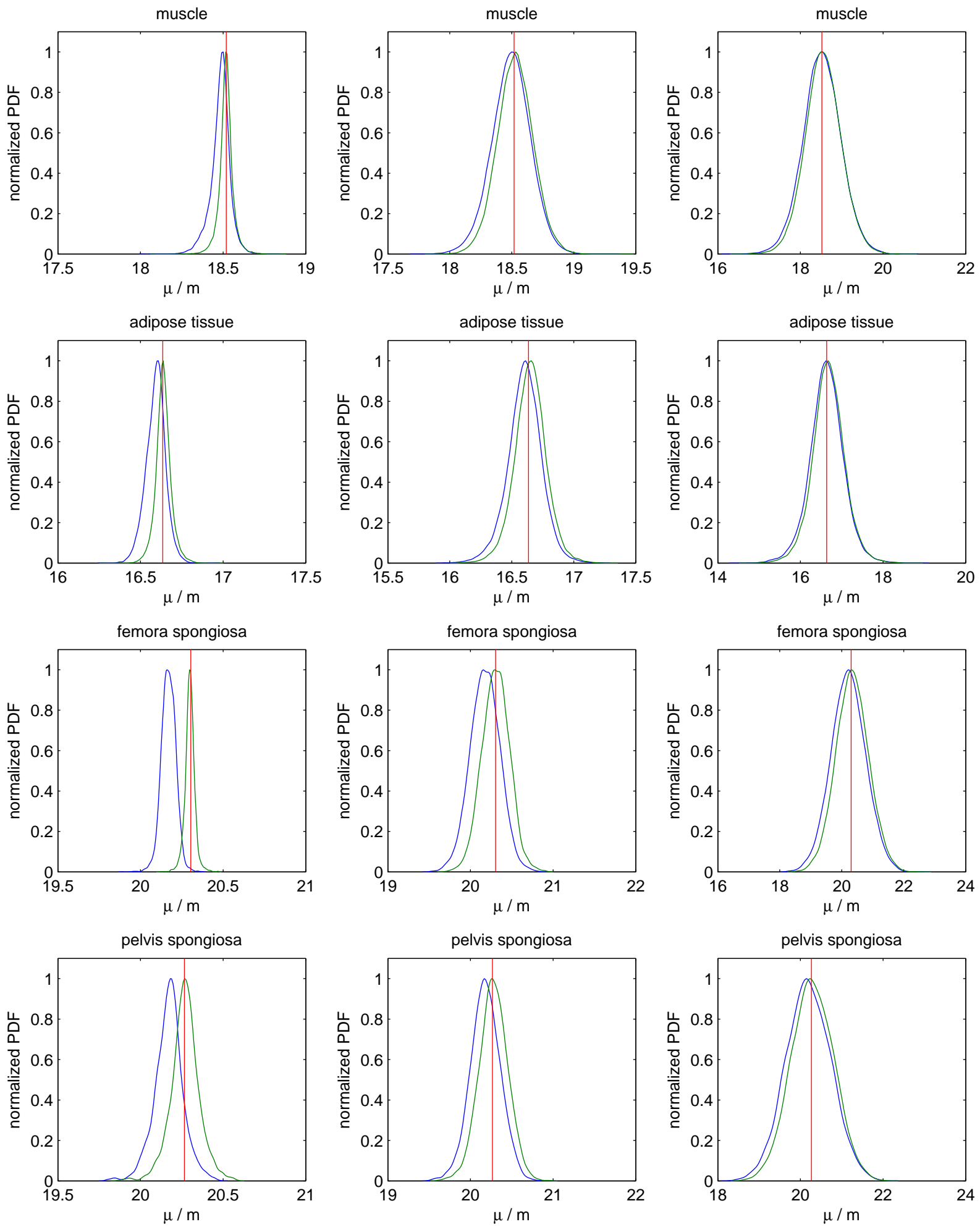

FIG. 9. Same as Fig. 4 for slice $\mathrm{C}$ and $\mu$ at $88 \mathrm{keV}$. 


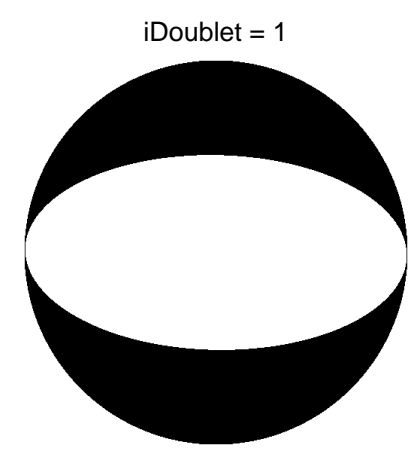

iDoublet $=1$

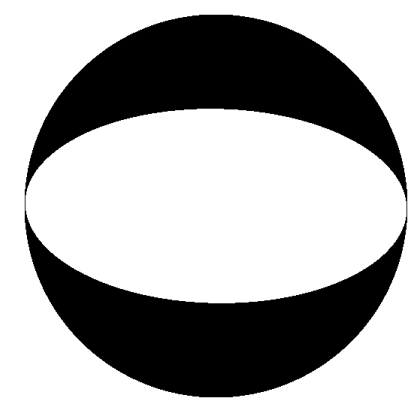

iDoublet $=1$

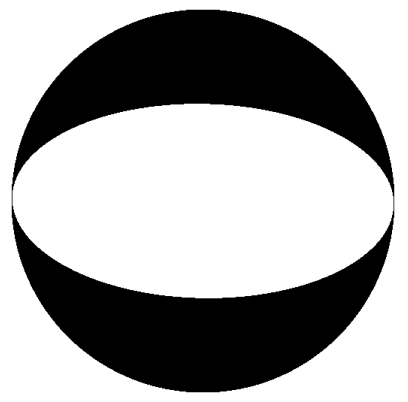

iDoublet $=2$

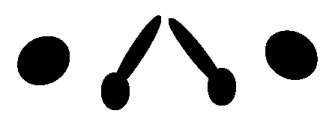

iDoublet $=2$

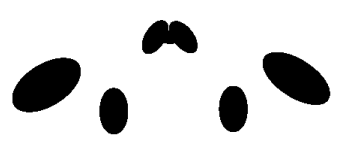

iDoublet $=2$

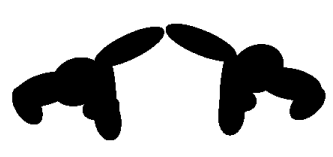

iTriplet $=1$

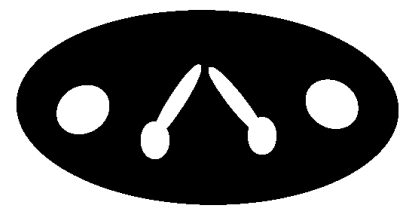

iTriplet $=1$

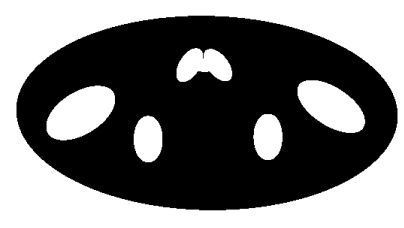

iTriplet $=1$

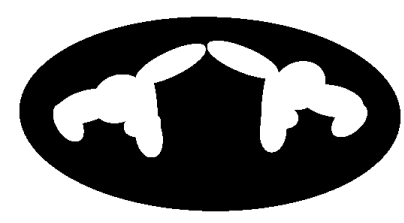

FIG. 10. Masks determined by DIRA for slices A (top row), B (middle row) and C (bottom row) for the 8th iteration and noise level $L=0$. The masks were produced by automatic segmentation of the $\mu$-images to outside air (iDoublet=1), bone (iDoublet=2) and soft tissue (iTriplet=1). We recall that the outside air was decomposed to the (lipid, water) doublet, bone was decomposed to the (compact bone, bone marrow) doublet and the soft tissue was decomposed to the (lipid, protein, water) triplet. The main purpose of the outside air doublet was to reduce partial volume artifacts at the soft tissue - outside air border. The masks were recalculated at each iteration of DIRA. 

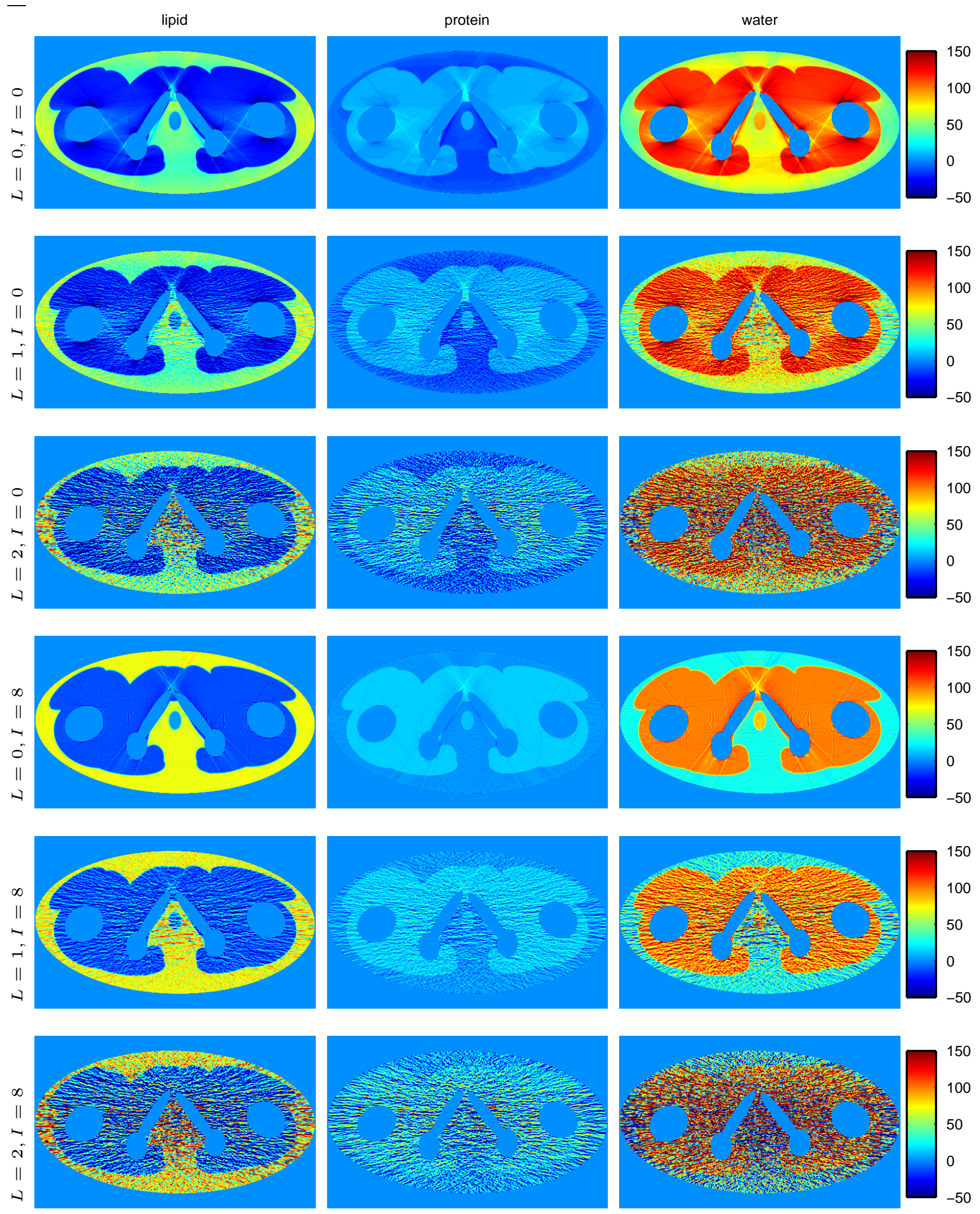

FIG. 11. Mass fractions of lipid, protein and water for noise levels $L=0,1,2$, iterations $I=0,8$, and slice A. 

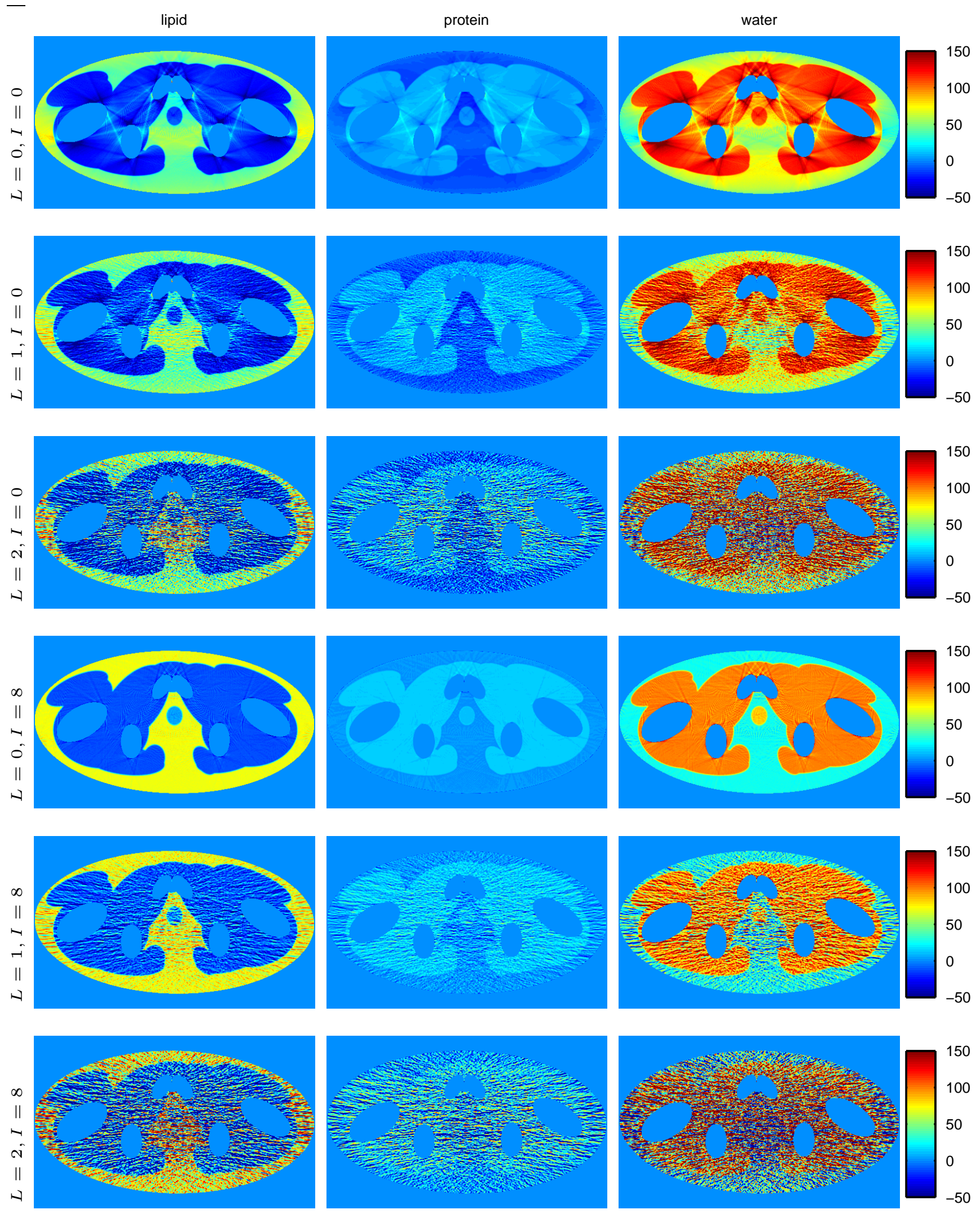

FIG. 12. Same as Fig. 11 for slice B. 

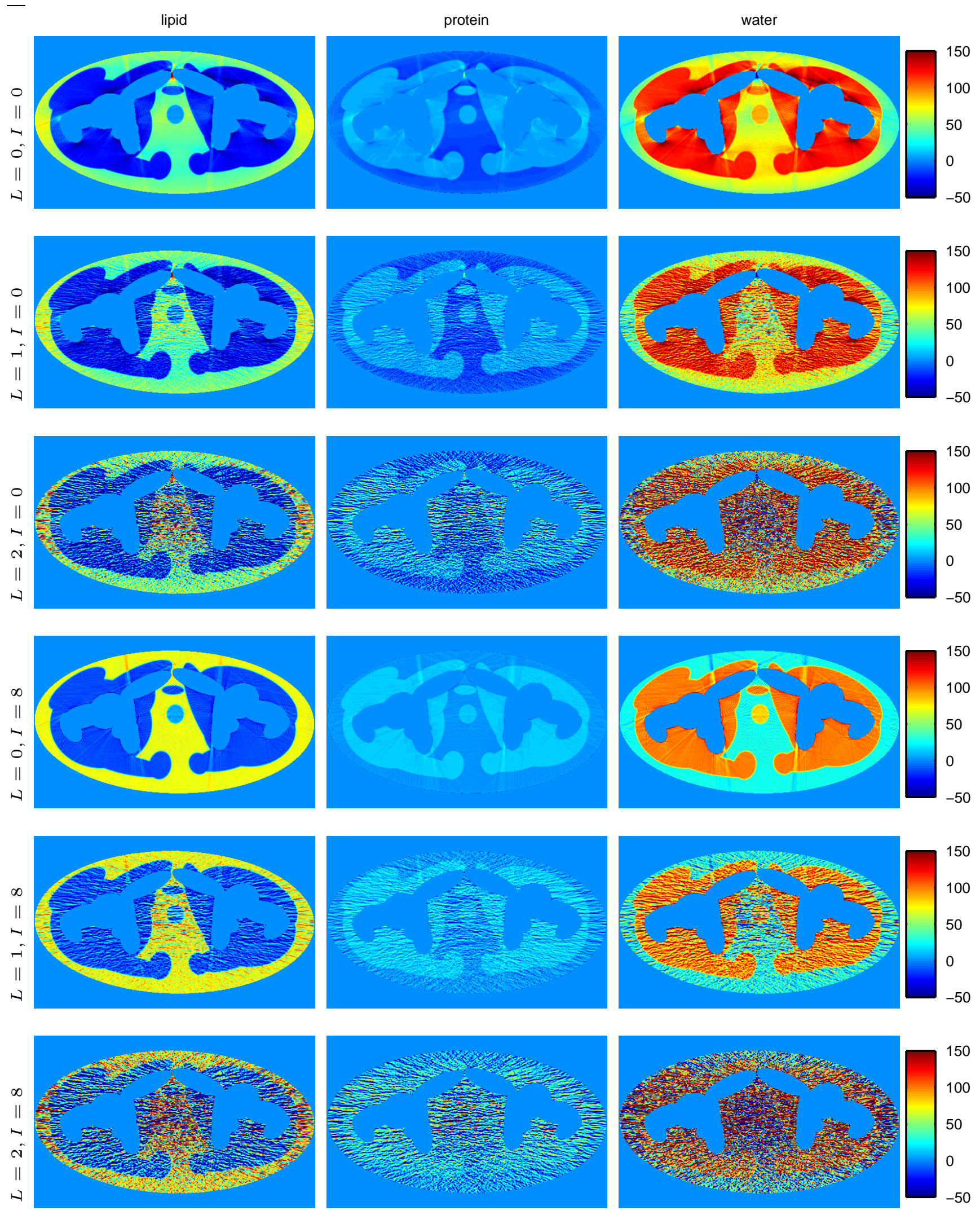

FIG. 13. Same as Fig. 11 for slice C. 

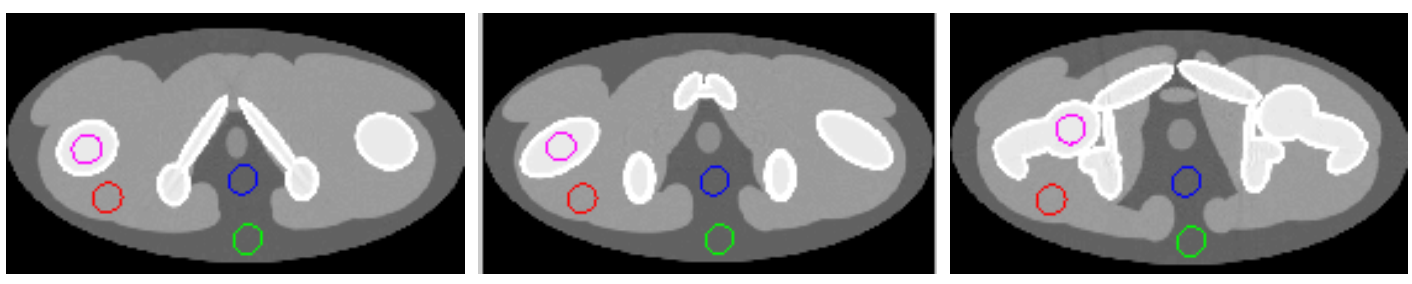

FIG. 14. Circular regions of interest: $R_{\mathrm{M}}$ (red) in muscle, $R_{\mathrm{A}}$ (green) in adipose tissue, $R_{\mathrm{C}}$ (blue) in adipose tissue and $R_{\mathrm{F}}$ (magenta) in femora spongiosa for slices A, B and C.
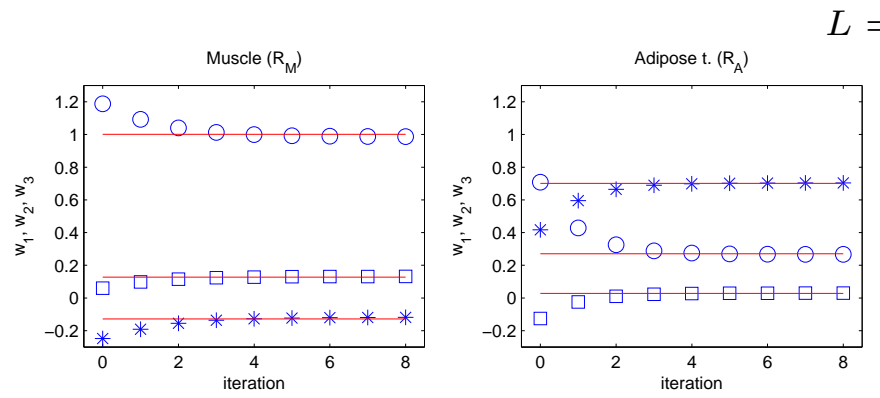

$L=0$
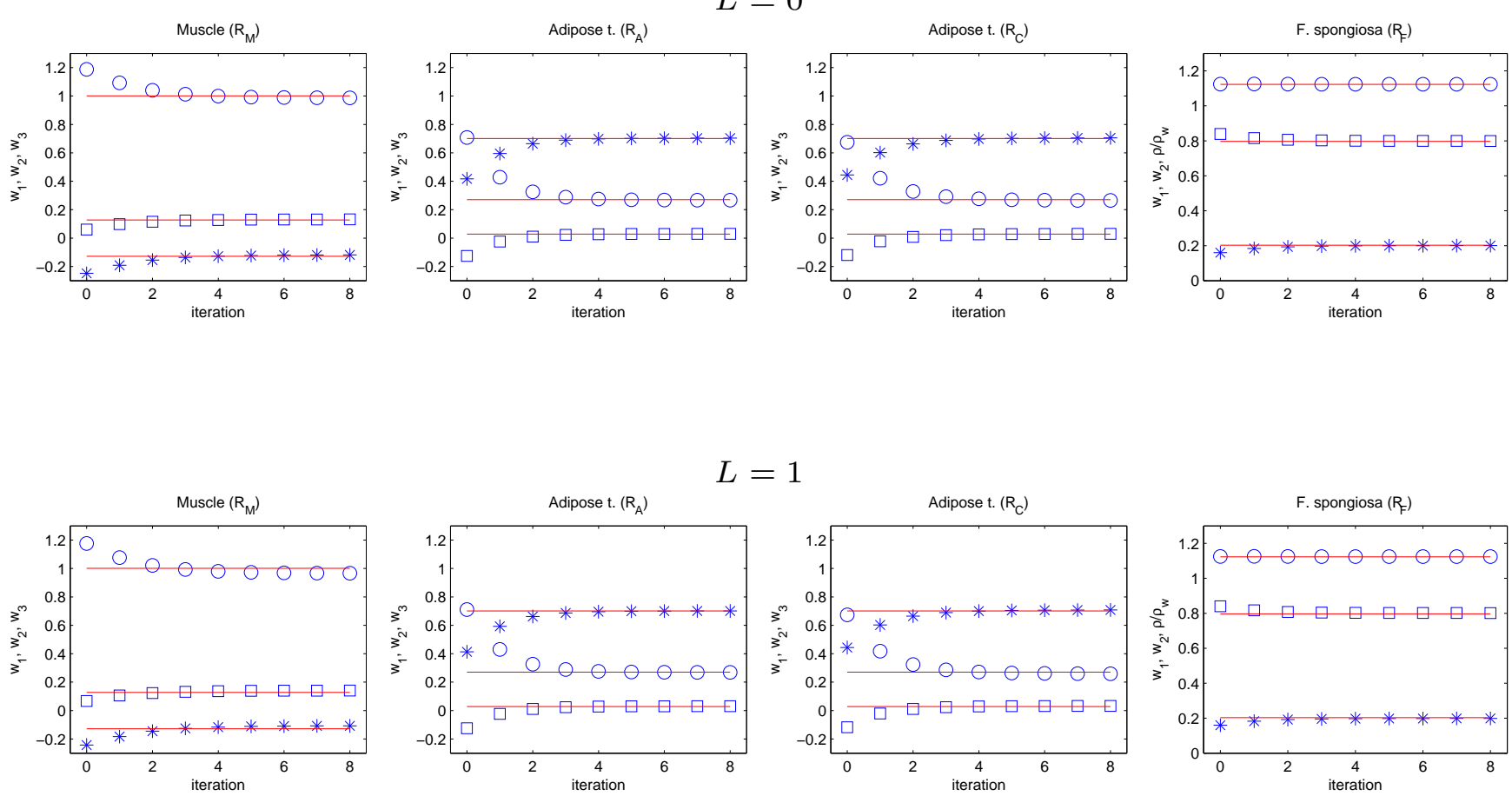

$L=1$
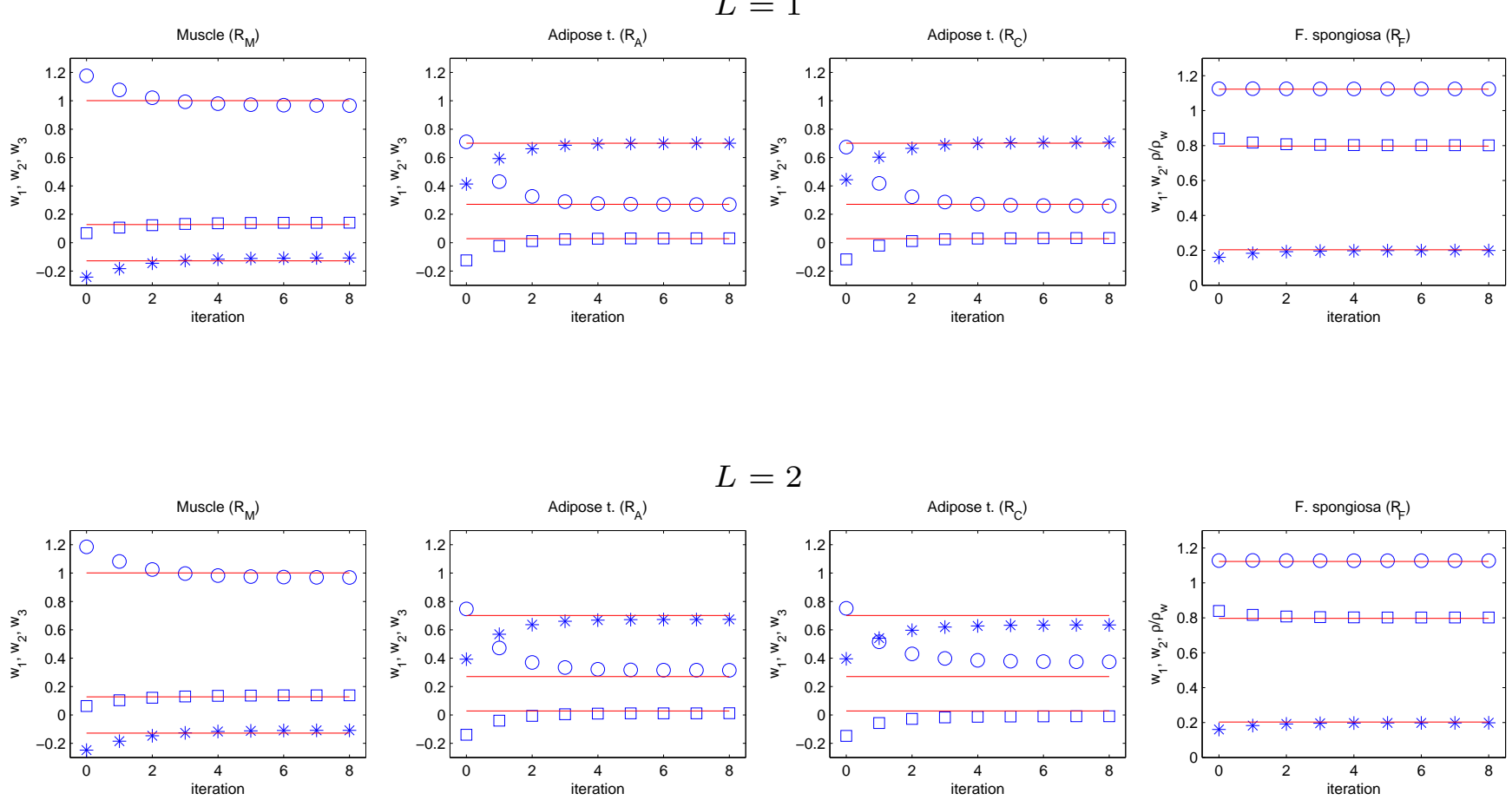

$L=2$
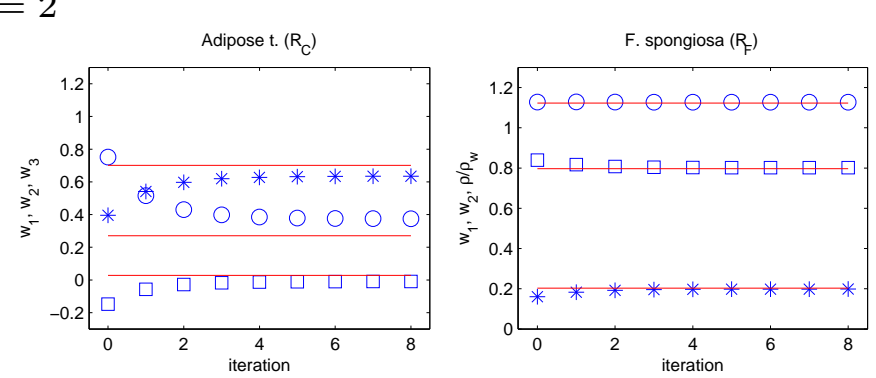

FIG. 15. Mass fractions of lipid $w_{1}(*)$, protein $w_{2}(\square)$ and water $w_{3}(\circ)$ as functions of the iteration number for muscle in region $R_{\mathrm{M}}$ and adipose tissue in regions $R_{\mathrm{A}}$ and $R_{\mathrm{C}}$. Mass fractions of compact bone $w_{1}(*)$ and bone marrow $w_{2}(\square)$ and mass density relative to water $\rho / \rho_{w}(\circ)$ for femora spongiosa in region $R_{\mathrm{F}}$. True values are plotted with horizontal solid lines. Noise level $L=0,1,2$ and slice A were used. 

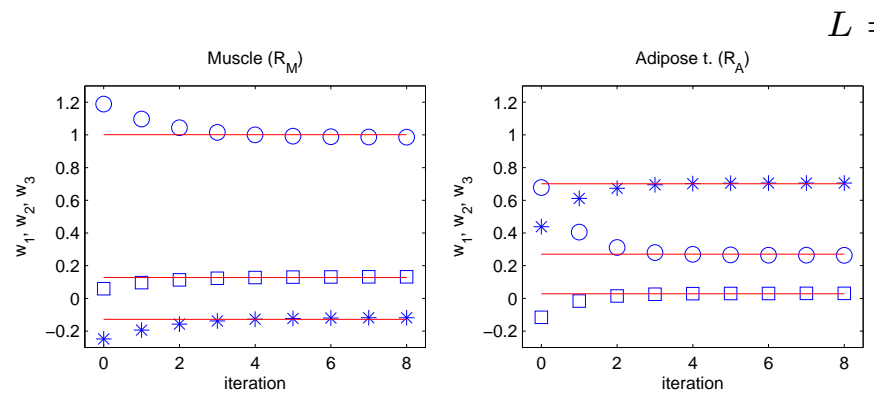

$$
L=0
$$
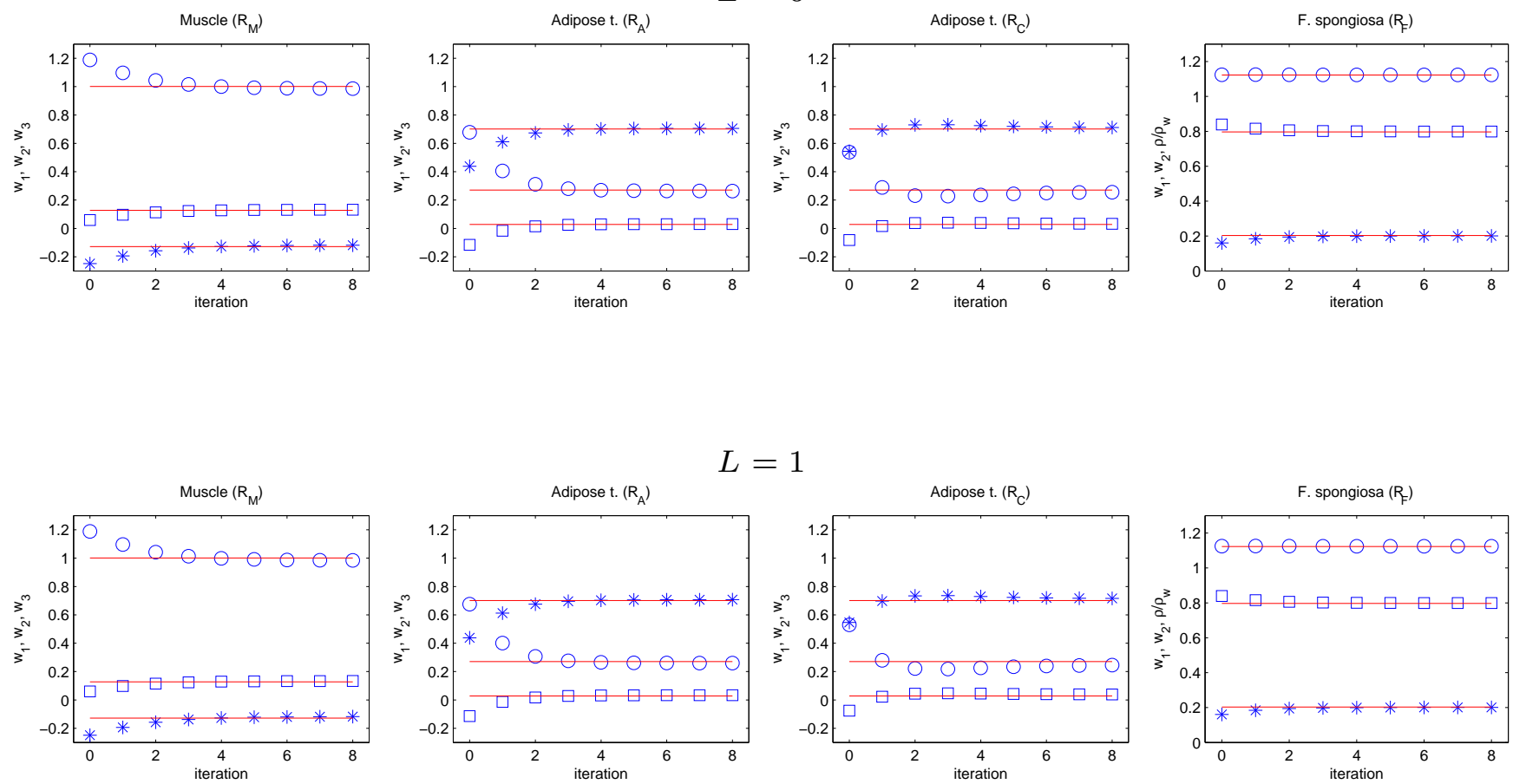

$$
L=1
$$
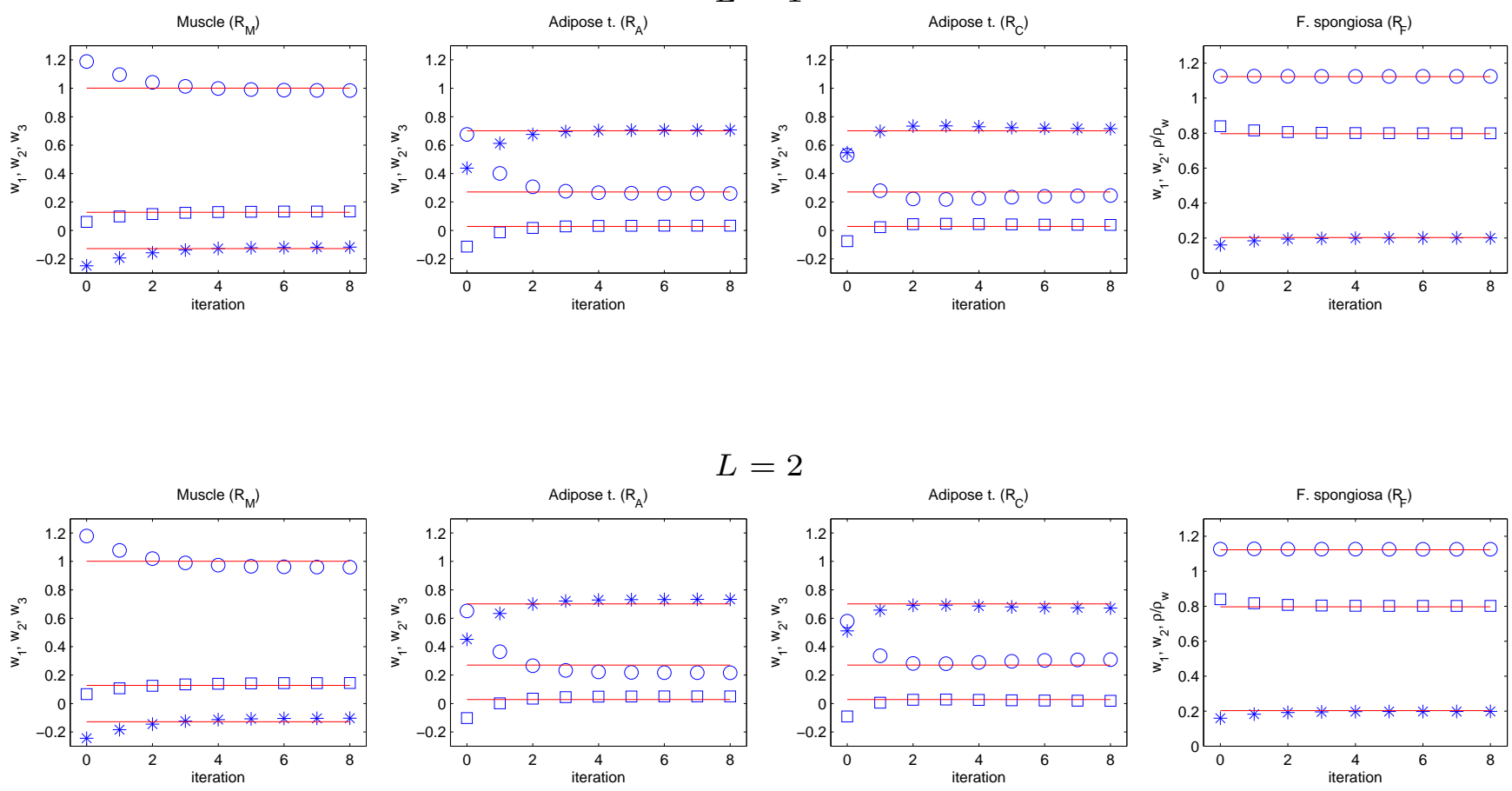

$$
L=2
$$
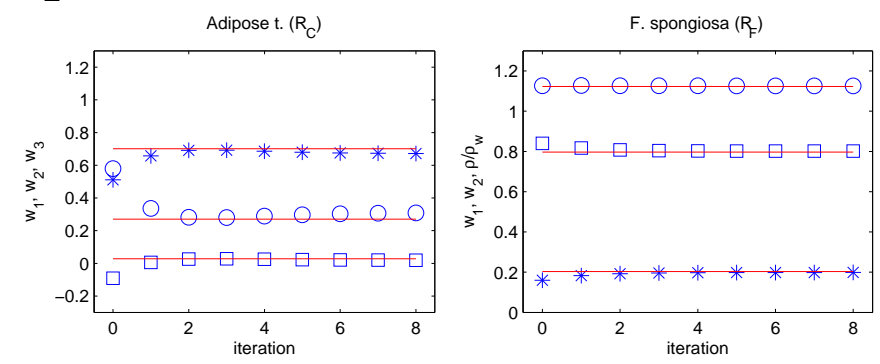

FIG. 16. Same as Fig. 16 for slice B. 

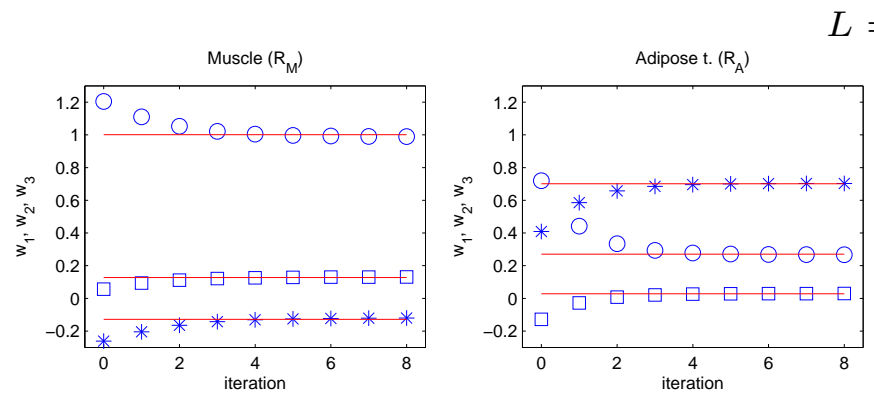

$$
L=0
$$
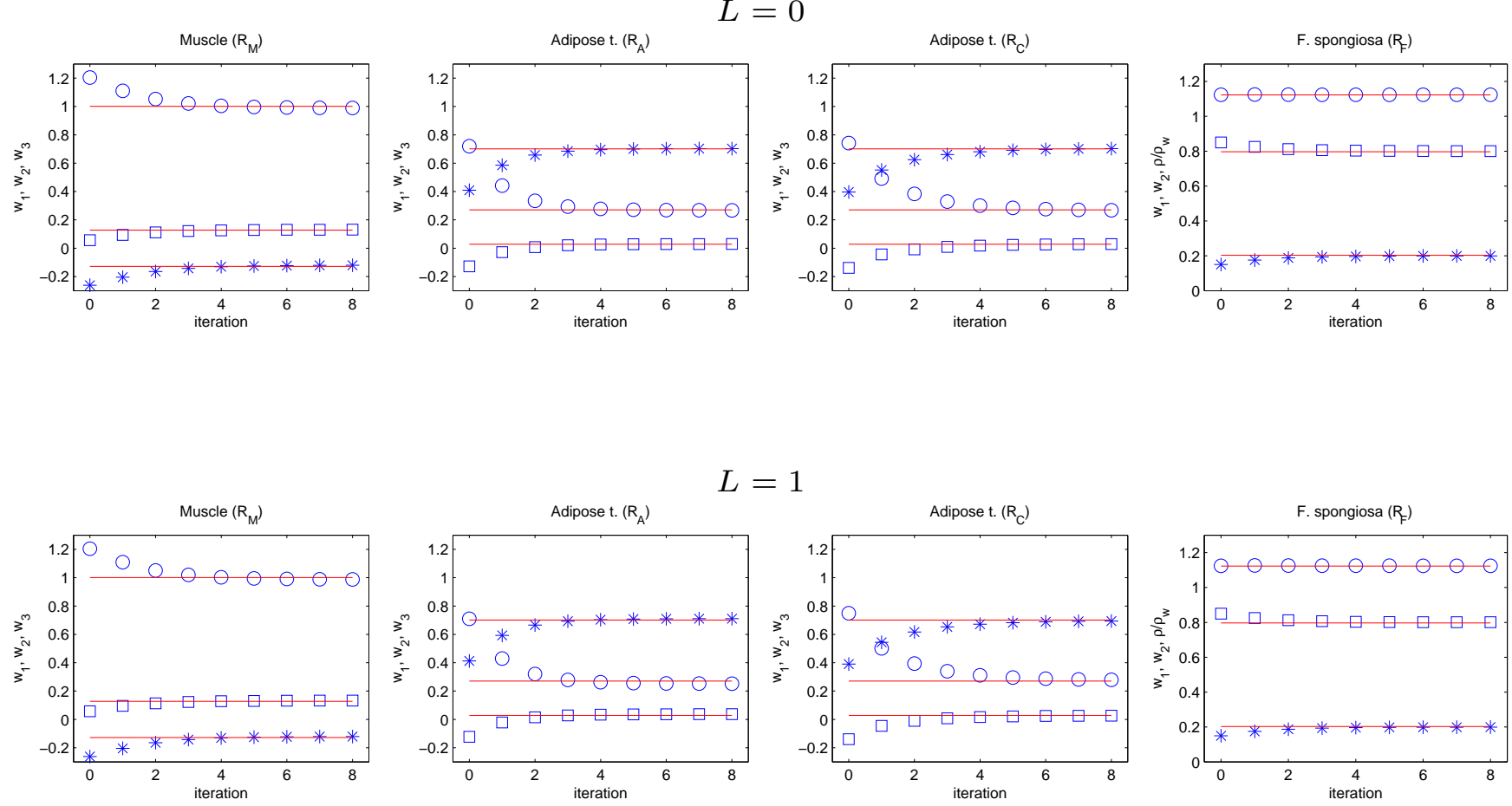

$$
L=1
$$
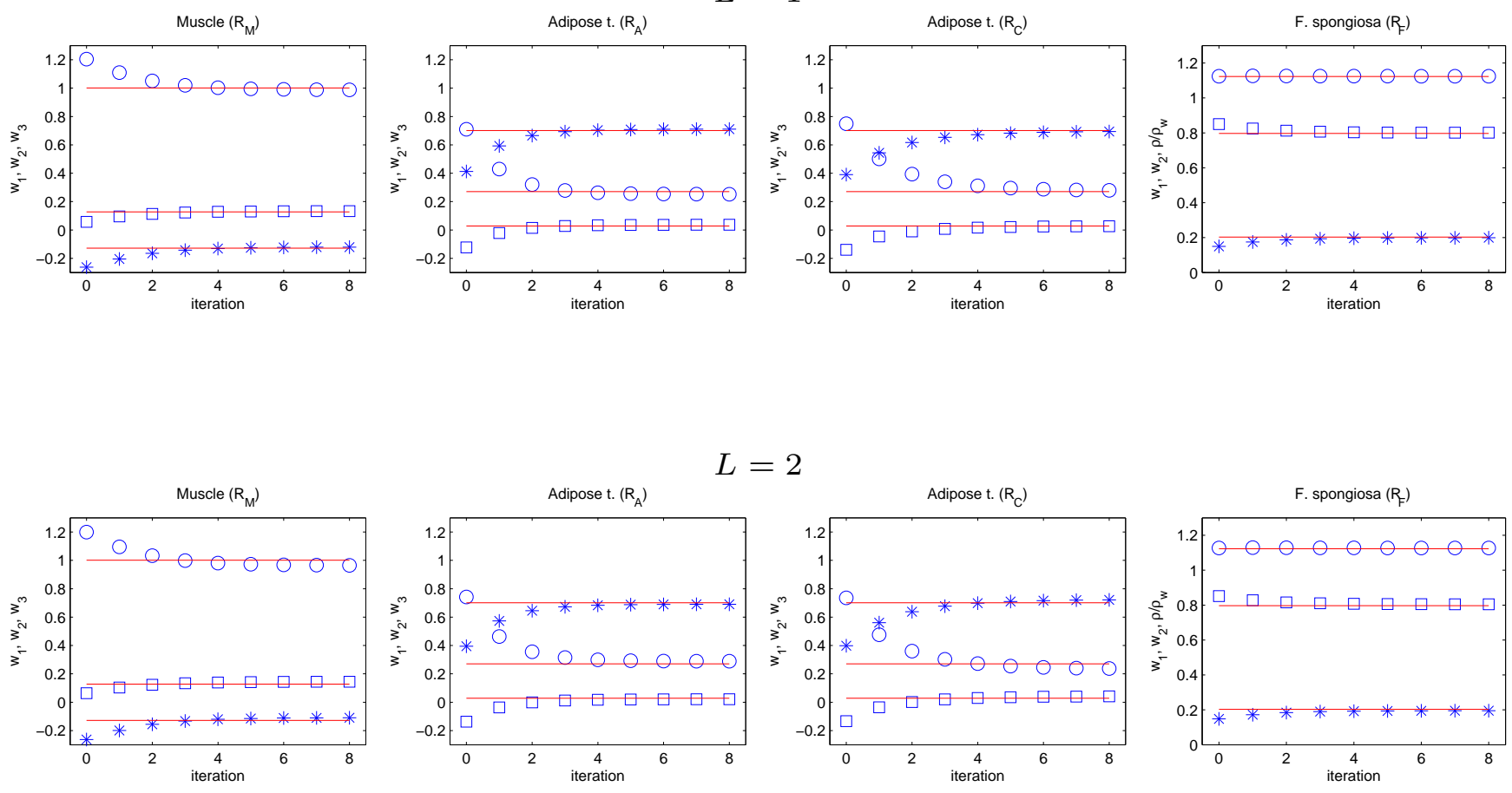

$$
L=2
$$
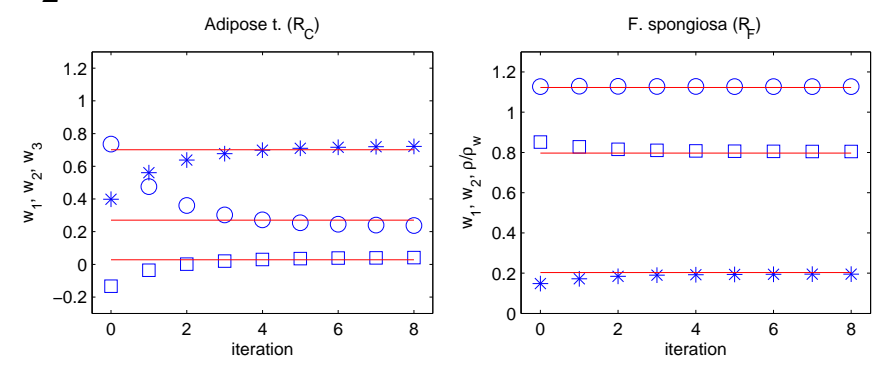

FIG. 17. Same as Fig. 16 for slice C. 
TABLE II. Relative error $\delta\left(\bar{\mu}_{i}\right)$ of average reconstructed linear attenuation coefficients $\bar{\mu}_{i}$, relative standard uncertainty $u_{\mathrm{r}}\left(\mu_{i}\right)$ of the reconstructed linear attenuation coefficients $\mu_{i}$, standard uncertainty $u\left(H_{i}\right)$ of the corresponding CT number at the effective energy $E_{i}(i=1,2)$ for pixels in four circular regions $R$, three noise levels $L$, three slices $S$ and 8th iteration. The correlation coefficient $r\left(\mu_{1}, \mu_{2}\right)$ and covariance $C\left(\mu_{1}, \mu_{2}\right)$ are also listed. Values are rescaled using the factor $\%_{0}=10^{-3}$.

\begin{tabular}{|c|c|c|c|c|c|c|c|c|c|c|}
\hline$S$ & $R$ & $L$ & $\begin{array}{r}\delta\left(\bar{\mu}_{1}\right) \\
(\% 0)\end{array}$ & $\begin{array}{r}\delta\left(\bar{\mu}_{2}\right) \\
(\% 0)\end{array}$ & $\begin{array}{r}u_{\mathrm{r}}\left(\mu_{1}\right) \\
(\% \mathrm{oo})\end{array}$ & $\begin{array}{r}u_{\mathrm{r}}\left(\mu_{2}\right) \\
(\% \mathrm{oo})\end{array}$ & $u\left(H_{1}\right)$ & $u\left(H_{2}\right)$ & $r\left(\mu_{1}, \mu_{2}\right)$ & $\begin{array}{r}C\left(\mu_{1}, \mu_{2}\right) \\
\left(\mathrm{cm}^{-2}\right)\end{array}$ \\
\hline $\mathrm{A}$ & $R_{\mathrm{M}}$ & 0 & -0.93 & -0.13 & 1.40 & 0.69 & 1 & 1 & 0.96 & $0.41 \times 10^{-3}$ \\
\hline $\mathrm{A}$ & $R_{\mathrm{M}}$ & 1 & -1.29 & 0.68 & 11.80 & 6.21 & 12 & 6 & -0.09 & $-2.97 \times 10^{-3}$ \\
\hline A & $R_{\mathrm{M}}$ & 2 & -2.05 & 1.01 & 32.05 & 20.94 & 33 & 22 & -0.05 & $-16.02 \times 10^{-3}$ \\
\hline A & $R_{\mathrm{A}}$ & 0 & -0.23 & -0.00 & 3.75 & 1.90 & 3 & 2 & 0.99 & $2.37 \times 10^{-3}$ \\
\hline A & $R_{\mathrm{A}}$ & 1 & 0.27 & 0.43 & 8.00 & 5.55 & 7 & 5 & 0.02 & $0.27 \times 10^{-3}$ \\
\hline A & $R_{\mathrm{A}}$ & 2 & 1.87 & 0.10 & 21.70 & 17.32 & 19 & 16 & -0.19 & $-24.41 \times 10^{-3}$ \\
\hline A & $R_{\mathrm{C}}$ & 0 & -0.53 & -0.17 & 4.13 & 2.00 & 4 & 2 & 0.99 & $2.76 \times 10^{-3}$ \\
\hline A & $R_{\mathrm{C}}$ & 1 & -0.68 & 0.32 & 17.51 & 9.63 & 16 & 9 & -0.14 & $-8.01 \times 10^{-3}$ \\
\hline A & $R_{\mathrm{C}}$ & 2 & 3.56 & 0.89 & 56.38 & 28.47 & 50 & 27 & -0.18 & $-99.34 \times 10^{-3}$ \\
\hline A & $R_{\mathrm{F}}$ & 0 & -1.57 & -0.22 & 0.94 & 0.50 & 1 & 1 & 0.96 & $0.27 \times 10^{-3}$ \\
\hline A & $R_{\mathrm{F}}$ & 1 & -2.50 & 0.27 & 12.95 & 6.79 & 16 & 8 & 0.00 & $0.03 \times 10^{-3}$ \\
\hline A & $R_{\mathrm{F}}$ & 2 & -1.34 & 2.65 & 43.57 & 24.28 & 55 & 28 & -0.01 & $-5.48 \times 10^{-3}$ \\
\hline B & $R_{\mathrm{M}}$ & 0 & -1.01 & -0.17 & 1.43 & 0.69 & 1 & 1 & 0.94 & $0.41 \times 10^{-3}$ \\
\hline B & $R_{\mathrm{M}}$ & 1 & -0.84 & 0.24 & 11.27 & 6.77 & 12 & 7 & -0.30 & $-10.22 \times 10^{-3}$ \\
\hline B & $R_{\mathrm{M}}$ & 2 & -1.96 & 1.89 & 29.76 & 23.24 & 31 & 24 & -0.18 & $-55.15 \times 10^{-3}$ \\
\hline B & $R_{\mathrm{A}}$ & 0 & -0.48 & -0.06 & 2.78 & 1.43 & 2 & 1 & 0.99 & $1.32 \times 10^{-3}$ \\
\hline B & $R_{\mathrm{A}}$ & 1 & -0.26 & 0.43 & 7.47 & 5.84 & 7 & 5 & -0.06 & $-0.88 \times 10^{-3}$ \\
\hline B & $R_{\mathrm{A}}$ & 2 & -2.39 & 1.57 & 21.19 & 16.77 & 19 & 16 & -0.20 & $-23.78 \times 10^{-3}$ \\
\hline B & $R_{\mathrm{C}}$ & 0 & -1.18 & -0.25 & 5.11 & 2.54 & 5 & 2 & 0.99 & $4.33 \times 10^{-3}$ \\
\hline B & $R_{\mathrm{C}}$ & 1 & -1.05 & 0.74 & 17.12 & 10.67 & 15 & 10 & -0.04 & $-2.27 \times 10^{-3}$ \\
\hline B & $R_{\mathrm{C}}$ & 2 & 1.79 & 2.74 & 62.62 & 30.36 & 56 & 28 & 0.01 & $4.83 \times 10^{-3}$ \\
\hline B & $R_{\mathrm{F}}$ & 0 & -1.27 & -0.13 & 1.03 & 0.56 & 1 & 1 & 0.96 & $0.33 \times 10^{-3}$ \\
\hline B & $R_{\mathrm{F}}$ & 1 & -0.68 & 0.63 & 13.10 & 7.35 & 17 & 8 & -0.22 & $-12.57 \times 10^{-3}$ \\
\hline B & $R_{\mathrm{F}}$ & 2 & -2.78 & 1.54 & 44.88 & 24.09 & 57 & 28 & -0.22 & $-135.34 \times 10^{-3}$ \\
\hline $\mathrm{C}$ & $R_{\mathrm{M}}$ & 0 & -0.74 & -0.11 & 2.65 & 1.31 & 3 & 1 & 0.97 & $1.48 \times 10^{-3}$ \\
\hline $\mathrm{C}$ & $R_{\mathrm{M}}$ & 1 & -0.42 & 0.47 & 10.35 & 8.37 & 11 & 9 & -0.21 & $-7.88 \times 10^{-3}$ \\
\hline $\mathrm{C}$ & $R_{\mathrm{M}}$ & 2 & -0.86 & 2.47 & 32.02 & 21.37 & 33 & 22 & -0.15 & $-45.26 \times 10^{-3}$ \\
\hline $\mathrm{C}$ & $R_{\mathrm{A}}$ & 0 & -0.17 & 0.01 & 3.74 & 1.93 & 3 & 2 & 0.99 & $2.41 \times 10^{-3}$ \\
\hline $\mathrm{C}$ & $R_{\mathrm{A}}$ & 1 & -0.45 & 0.67 & 7.72 & 5.99 & 7 & 6 & -0.04 & $-0.61 \times 10^{-3}$ \\
\hline $\mathrm{C}$ & $R_{\mathrm{A}}$ & 2 & 0.24 & 0.23 & 22.40 & 18.18 & 20 & 17 & -0.30 & $-40.64 \times 10^{-3}$ \\
\hline $\mathrm{C}$ & $R_{\mathrm{C}}$ & 0 & -0.25 & -0.10 & 6.99 & 3.44 & 6 & 3 & 1.00 & $8.06 \times 10^{-3}$ \\
\hline $\mathrm{C}$ & $R_{\mathrm{C}}$ & 1 & 0.81 & 0.52 & 18.24 & 9.92 & 16 & 9 & 0.07 & $4.08 \times 10^{-3}$ \\
\hline $\mathrm{C}$ & $R_{\mathrm{C}}$ & 2 & -3.75 & 2.30 & 52.61 & 31.95 & 47 & 30 & -0.30 & $-167.37 \times 10^{-3}$ \\
\hline $\mathrm{C}$ & $R_{\mathrm{F}}$ & 0 & -2.30 & -0.32 & 2.07 & 1.09 & 3 & 1 & 0.96 & $1.25 \times 10^{-3}$ \\
\hline $\mathrm{C}$ & $R_{\mathrm{F}}$ & 1 & -1.84 & 0.67 & 13.99 & 9.46 & 18 & 11 & -0.11 & $-8.30 \times 10^{-3}$ \\
\hline $\mathrm{C}$ & $R_{\mathrm{F}}$ & 2 & -4.48 & 2.12 & 44.71 & 28.63 & 56 & 33 & -0.15 & $-112.83 \times 10^{-3}$ \\
\hline
\end{tabular}


TABLE III. Errors of average mass fractions of lipid $\epsilon\left(\bar{w}_{1}\right)$, protein $\epsilon\left(\bar{w}_{2}\right)$ and water $\epsilon\left(\bar{w}_{3}\right)$, and the relative error of average mass density of the mixture $\delta(\bar{\rho})$ for slices $S$, regions $R$, noise levels $L$ and 8th iteration. Also shown are standard uncertainties $u\left(w_{i}\right)$ and $u(\rho)$ in mass fractions and mass density, respectively.

\begin{tabular}{|c|c|c|c|c|c|c|c|c|c|c|}
\hline$S$ & $R$ & $L$ & $\begin{array}{r}\epsilon\left(\bar{w}_{1}\right) \\
(\%)\end{array}$ & $\begin{array}{r}\epsilon\left(\bar{w}_{2}\right) \\
(\%)\end{array}$ & $\begin{array}{r}\epsilon\left(\bar{w}_{3}\right) \\
(\%)\end{array}$ & $\begin{array}{r}\delta(\bar{\rho}) \\
(\%)\end{array}$ & $\begin{array}{r}u\left(w_{1}\right) \\
(\%)\end{array}$ & $\begin{array}{r}u\left(w_{2}\right) \\
(\%)\end{array}$ & $\begin{array}{r}u\left(w_{3}\right) \\
(\%)\end{array}$ & $\begin{array}{r}u(\rho) \\
\left(\mathrm{g} \mathrm{cm}^{-3}\right)\end{array}$ \\
\hline A & $R_{\mathrm{M}}$ & 0 & 0.98 & 0.42 & -1.41 & -0.33 & 1.01 & 0.27 & 1.25 & $0.05 \times 10^{-2}$ \\
\hline A & $R_{\mathrm{M}}$ & 1 & 2.09 & 1.31 & -3.40 & -0.17 & 16.02 & 9.61 & 25.42 & $1.32 \times 10^{-2}$ \\
\hline A & $R_{\mathrm{M}}$ & 2 & 2.01 & 1.11 & -3.13 & -0.08 & 45.10 & 28.53 & 72.89 & $4.17 \times 10^{-2}$ \\
\hline A & $R_{\mathrm{A}}$ & 0 & 0.24 & 0.12 & -0.37 & -0.08 & 2.40 & 0.37 & 2.71 & $0.13 \times 10^{-2}$ \\
\hline $\mathrm{A}$ & $R_{\mathrm{A}}$ & 1 & 0.00 & 0.19 & -0.20 & -0.03 & 9.89 & 6.96 & 16.58 & $0.95 \times 10^{-2}$ \\
\hline A & $R_{\mathrm{A}}$ & 2 & -2.80 & -1.68 & 4.48 & -0.17 & 30.88 & 22.98 & 53.27 & $3.10 \times 10^{-2}$ \\
\hline $\mathrm{A}$ & $R_{\mathrm{C}}$ & 0 & 0.42 & 0.13 & -0.55 & -0.09 & 2.73 & 0.45 & 3.13 & $0.13 \times 10^{-2}$ \\
\hline A & $R_{\mathrm{C}}$ & 1 & 0.67 & 0.43 & -1.10 & -0.00 & 21.81 & 14.49 & 35.91 & $1.83 \times 10^{-2}$ \\
\hline A & $R_{\mathrm{C}}$ & 2 & -6.66 & -3.77 & 10.43 & -0.12 & 71.45 & 46.40 & 116.79 & $5.62 \times 10^{-2}$ \\
\hline B & $R_{\mathrm{M}}$ & 0 & 1.04 & 0.44 & -1.48 & -0.33 & 1.08 & 0.32 & 1.36 & $0.05 \times 10^{-2}$ \\
\hline B & $R_{\mathrm{M}}$ & 1 & 1.04 & 0.59 & -1.63 & -0.27 & 17.00 & 10.69 & 27.54 & $1.50 \times 10^{-2}$ \\
\hline B & $R_{\mathrm{M}}$ & 2 & 2.50 & 1.65 & -4.15 & 0.06 & 46.44 & 30.98 & 76.79 & $4.67 \times 10^{-2}$ \\
\hline B & $R_{\mathrm{A}}$ & 0 & 0.46 & 0.21 & -0.67 & -0.08 & 1.76 & 0.24 & 1.96 & $0.10 \times 10^{-2}$ \\
\hline B & $R_{\mathrm{A}}$ & 1 & 0.58 & 0.49 & -1.07 & -0.01 & 9.86 & 7.26 & 16.87 & $1.01 \times 10^{-2}$ \\
\hline B & $R_{\mathrm{A}}$ & 2 & 3.17 & 2.19 & -5.37 & 0.29 & 29.46 & 21.91 & 50.80 & $3.01 \times 10^{-2}$ \\
\hline B & $R_{\mathrm{C}}$ & 0 & 1.07 & 0.42 & -1.49 & -0.07 & 3.31 & 0.49 & 3.75 & $0.17 \times 10^{-2}$ \\
\hline B & $R_{\mathrm{C}}$ & 1 & 1.46 & 1.04 & -2.50 & 0.08 & 21.08 & 14.39 & 34.97 & $1.89 \times 10^{-2}$ \\
\hline B & $R_{\mathrm{C}}$ & 2 & -2.94 & -0.90 & 3.84 & 0.28 & 73.69 & 46.26 & 118.49 & $5.61 \times 10^{-2}$ \\
\hline $\mathrm{C}$ & $R_{\mathrm{M}}$ & 0 & 0.78 & 0.34 & -1.11 & -0.34 & 1.89 & 0.47 & 2.30 & $0.10 \times 10^{-2}$ \\
\hline $\mathrm{C}$ & $R_{\mathrm{M}}$ & 1 & 0.76 & 0.53 & -1.29 & -0.26 & 16.54 & 11.12 & 27.45 & $1.68 \times 10^{-2}$ \\
\hline $\mathrm{C}$ & $R_{\mathrm{M}}$ & 2 & 1.89 & 1.69 & -3.58 & 0.10 & 47.19 & 30.13 & 76.68 & $4.39 \times 10^{-2}$ \\
\hline $\mathrm{C}$ & $R_{\mathrm{A}}$ & 0 & 0.20 & 0.11 & -0.30 & -0.08 & 2.36 & 0.30 & 2.61 & $0.13 \times 10^{-2}$ \\
\hline $\mathrm{C}$ & $R_{\mathrm{A}}$ & 1 & 0.99 & 0.83 & -1.82 & 0.04 & 10.07 & 7.39 & 17.20 & $1.03 \times 10^{-2}$ \\
\hline $\mathrm{C}$ & $R_{\mathrm{A}}$ & 2 & -1.08 & -0.76 & 1.84 & -0.07 & 32.97 & 24.64 & 57.09 & $3.34 \times 10^{-2}$ \\
\hline $\mathrm{C}$ & $R_{\mathrm{C}}$ & 0 & 0.19 & 0.04 & -0.23 & -0.10 & 4.55 & 0.64 & 5.15 & $0.22 \times 10^{-2}$ \\
\hline $\mathrm{C}$ & $R_{\mathrm{C}}$ & 1 & -0.71 & -0.18 & 0.89 & -0.04 & 21.10 & 13.62 & 34.20 & $1.73 \times 10^{-2}$ \\
\hline $\mathrm{C}$ & $R_{\mathrm{C}}$ & 2 & 2.05 & 1.25 & -3.30 & 0.47 & 70.38 & 48.66 & 118.05 & $6.23 \times 10^{-2}$ \\
\hline
\end{tabular}


TABLE IV. Relative errors of average mass fractions of lipid $\delta\left(\bar{w}_{1}\right)$, protein $\delta\left(\bar{w}_{2}\right)$ and water $\delta\left(\bar{w}_{3}\right)$ and corresponding average mass fractions $\bar{w}_{1}, \bar{w}_{2}$ and $\bar{w}_{3}$ for slices $S$, regions $R$, noise levels $L$ and 8th iteration.

\begin{tabular}{|c|c|c|c|c|c|c|c|c|}
\hline$S$ & $R$ & $L$ & $\bar{w}_{1}$ & $\begin{array}{r}\delta\left(\bar{w}_{1}\right) \\
(\%)\end{array}$ & $\bar{w}_{2}$ & $\begin{array}{r}\delta\left(\bar{w}_{2}\right) \\
(\%)\end{array}$ & $\bar{w}_{3}$ & $\begin{array}{r}\delta\left(\bar{w}_{3}\right) \\
(\%)\end{array}$ \\
\hline $\mathrm{A}$ & $R_{\mathrm{M}}$ & 0 & -0.119 & -7.6 & 0.132 & 3.3 & 0.987 & -1.4 \\
\hline A & $R_{\mathrm{M}}$ & 2 & -0.108 & -15.7 & 0.139 & 8.7 & 0.970 & -3.1 \\
\hline A & $R_{\mathrm{A}}$ & 0 & 0.704 & 0.3 & 0.030 & 4.3 & 0.267 & -1.4 \\
\hline A & $R_{\mathrm{C}}$ & 0 & 0.705 & 0.6 & 0.030 & 4.5 & 0.265 & -2.0 \\
\hline A & $R_{\mathrm{C}}$ & 1 & 0.708 & 1.0 & 0.033 & 15.0 & 0.259 & -4.1 \\
\hline A & $R_{\mathrm{C}}$ & 2 & 0.635 & -9.5 & -0.009 & -132.3 & 0.375 & 38.6 \\
\hline B & $R_{\mathrm{M}}$ & 0 & -0.118 & -8.1 & 0.132 & 3.4 & 0.986 & -1.5 \\
\hline B & $R_{\mathrm{A}}$ & 1 & 0.707 & 0.8 & 0.033 & 17.3 & 0.260 & -4.0 \\
\hline B & $R_{\mathrm{A}}$ & 2 & 0.733 & 4.5 & 0.050 & 77.1 & 0.217 & -19.9 \\
\hline B & $R_{\mathrm{C}}$ & 0 & 0.712 & 1.5 & 0.033 & 14.9 & 0.255 & -5.5 \\
\hline B & $R_{\mathrm{C}}$ & 1 & 0.716 & 2.1 & 0.039 & 36.5 & 0.245 & -9.2 \\
\hline B & $R_{\mathrm{C}}$ & 2 & 0.672 & -4.2 & 0.019 & -31.6 & 0.309 & 14.2 \\
\hline $\mathrm{C}$ & $R_{\mathrm{M}}$ & 0 & -0.121 & -6.1 & 0.131 & 2.6 & 0.990 & -1.1 \\
\hline $\mathrm{C}$ & $R_{\mathrm{M}}$ & 1 & -0.121 & -5.9 & 0.133 & 4.2 & 0.988 & -1.3 \\
\hline $\mathrm{C}$ & $R_{\mathrm{M}}$ & 2 & -0.109 & -14.7 & 0.144 & 13.2 & 0.965 & -3.6 \\
\hline $\mathrm{C}$ & $R_{\mathrm{A}}$ & 0 & 0.703 & 0.3 & 0.030 & 3.7 & 0.267 & -1.1 \\
\hline
\end{tabular}


TABLE V. Correlation coefficients between variables. Mass fractions of lipid, protein and water are denoted $w_{1}, w_{2}$ and $w_{3}$, respectively. Calculated mass density of the mixture is denoted $\rho$.

\begin{tabular}{|c|c|c|c|c|c|c|c|c|}
\hline$S$ & $R$ & $L$ & $r\left(w_{1}, w_{2}\right)$ & $r\left(w_{1}, w_{3}\right)$ & $r\left(w_{2}, w_{3}\right)$ & $r\left(w_{1}, \rho\right)$ & $r\left(w_{2}, \rho\right)$ & $r\left(w_{3}, \rho\right)$ \\
\hline $\mathrm{A}$ & $R_{\mathrm{M}}$ & 0 & 0.836 & -0.993 & -0.895 & -0.615 & -0.081 & 0.517 \\
\hline A & $R_{\mathrm{M}}$ & 1 & 0.967 & -0.995 & -0.987 & 0.846 & 0.954 & -0.894 \\
\hline A & $R_{\mathrm{M}}$ & 2 & 0.958 & -0.994 & -0.984 & 0.832 & 0.955 & -0.888 \\
\hline A & $R_{\mathrm{A}}$ & 0 & 0.786 & -0.996 & -0.836 & -0.912 & -0.463 & 0.874 \\
\hline A & $R_{\mathrm{A}}$ & 1 & 0.933 & -0.989 & -0.977 & 0.786 & 0.956 & -0.870 \\
\hline A & $R_{\mathrm{A}}$ & 2 & 0.955 & -0.992 & -0.985 & 0.863 & 0.973 & -0.920 \\
\hline A & $R_{\mathrm{C}}$ & 0 & 0.902 & -0.998 & -0.927 & -0.936 & -0.693 & 0.913 \\
\hline A & $R_{\mathrm{C}}$ & 1 & 0.956 & -0.993 & -0.984 & 0.838 & 0.962 & -0.897 \\
\hline A & $R_{\mathrm{C}}$ & 2 & 0.962 & -0.994 & -0.986 & 0.850 & 0.960 & -0.901 \\
\hline B & $R_{\mathrm{M}}$ & 0 & 0.847 & -0.992 & -0.907 & -0.468 & 0.074 & 0.354 \\
\hline B & $R_{\mathrm{M}}$ & 1 & 0.976 & -0.996 & -0.991 & 0.898 & 0.972 & -0.932 \\
\hline B & $R_{\mathrm{M}}$ & 2 & 0.966 & -0.995 & -0.988 & 0.873 & 0.968 & -0.918 \\
\hline B & $R_{\mathrm{A}}$ & 0 & 0.801 & -0.997 & -0.843 & -0.939 & -0.545 & 0.911 \\
\hline B & $R_{\mathrm{A}}$ & 1 & 0.941 & -0.989 & -0.980 & 0.820 & 0.965 & -0.895 \\
\hline B & $R_{\mathrm{A}}$ & 2 & 0.954 & -0.992 & -0.985 & 0.862 & 0.973 & -0.920 \\
\hline B & $R_{\mathrm{C}}$ & 0 & 0.880 & -0.998 & -0.908 & -0.946 & -0.678 & 0.924 \\
\hline B & $R_{\mathrm{C}}$ & 1 & 0.942 & -0.990 & -0.979 & 0.803 & 0.956 & -0.878 \\
\hline B & $R_{\mathrm{C}}$ & 2 & 0.949 & -0.992 & -0.981 & 0.792 & 0.942 & -0.860 \\
\hline $\mathrm{C}$ & $R_{\mathrm{M}}$ & 0 & 0.841 & -0.994 & -0.896 & -0.680 & -0.176 & 0.594 \\
\hline $\mathrm{C}$ & $R_{\mathrm{M}}$ & 1 & 0.968 & -0.995 & -0.989 & 0.881 & 0.971 & -0.924 \\
\hline $\mathrm{C}$ & $R_{\mathrm{M}}$ & 2 & 0.966 & -0.995 & -0.987 & 0.859 & 0.962 & -0.907 \\
\hline $\mathrm{C}$ & $R_{\mathrm{A}}$ & 0 & 0.819 & -0.998 & -0.855 & -0.954 & -0.610 & 0.932 \\
\hline $\mathrm{C}$ & $R_{\mathrm{A}}$ & 1 & 0.938 & -0.989 & -0.979 & 0.813 & 0.964 & -0.890 \\
\hline $\mathrm{C}$ & $R_{\mathrm{A}}$ & 2 & 0.963 & -0.993 & -0.988 & 0.886 & 0.977 & -0.933 \\
\hline $\mathrm{C}$ & $R_{\mathrm{C}}$ & 0 & 0.930 & -0.999 & -0.946 & -0.969 & -0.810 & 0.956 \\
\hline $\mathrm{C}$ & $R_{\mathrm{C}}$ & 1 & 0.936 & -0.990 & -0.976 & 0.766 & 0.943 & -0.848 \\
\hline $\mathrm{C}$ & $R_{\mathrm{C}}$ & 2 & 0.965 & -0.994 & -0.988 & 0.876 & 0.969 & -0.922 \\
\hline
\end{tabular}


TABLE VI. Errors of average mass fractions of mineral bone $\epsilon\left(\bar{w}_{1}\right)$ and bone marrow $\epsilon\left(\bar{w}_{2}\right)$, and relative error of average mass density of the mixture $\delta(\bar{\rho})$ for the slice $S$, region $R_{\mathrm{F}}$, noise levels $L$ and 8th iteration. Also shown are corresponding standard uncertainties $u\left(w_{1}\right), u\left(w_{2}\right)$ and $u(\rho)$ and the correlation coefficient $r\left(w_{1}, \rho\right)$.

\begin{tabular}{|c|c|c|c|c|c|c|c|}
\hline$S$ & $L$ & $\begin{array}{r}\epsilon\left(\bar{w}_{1}\right) \\
(\%)\end{array}$ & $\begin{array}{r}\epsilon\left(\bar{w}_{2}\right) \\
(\%)\end{array}$ & $\begin{array}{r}\delta(\bar{\rho}) \\
(\%)\end{array}$ & $\begin{array}{r}u\left(w_{1}\right) \\
(\%)\end{array}$ & $\begin{array}{r}u(\rho) \\
\left(\mathrm{g} \mathrm{cm}^{-3}\right)\end{array}$ & $r\left(w_{1}, \rho\right)$ \\
\hline A & 0 & -0.219 & 0.219 & 0.01 & 0.08 & $0.05 \times 10^{-2}$ & 0.735 \\
\hline A & 1 & -0.431 & 0.431 & 0.10 & 2.34 & $1.01 \times 10^{-2}$ & -0.746 \\
\hline A & 2 & -0.447 & 0.447 & 0.36 & 8.02 & $3.62 \times 10^{-2}$ & -0.753 \\
\hline B & 0 & -0.183 & 0.183 & 0.01 & 0.08 & $0.05 \times 10^{-2}$ & 0.760 \\
\hline B & 1 & -0.187 & 0.187 & 0.09 & 2.64 & $1.16 \times 10^{-2}$ & -0.833 \\
\hline B & 2 & -0.443 & 0.443 & 0.26 & 8.92 & $3.84 \times 10^{-2}$ & -0.828 \\
\hline $\mathrm{C}$ & 0 & -0.318 & 0.318 & 0.02 & 0.17 & $0.10 \times 10^{-2}$ & 0.739 \\
\hline $\mathrm{C}$ & 1 & -0.374 & 0.374 & 0.13 & 2.85 & $1.42 \times 10^{-2}$ & -0.808 \\
\hline $\mathrm{C}$ & 2 & -0.770 & 0.770 & 0.37 & 9.18 & $4.37 \times 10^{-2}$ & -0.816 \\
\hline
\end{tabular}

TABLE VII. Relative errors $\delta\left(\bar{w}_{1}\right)$ and $\delta\left(\bar{w}_{2}\right)$ of average mass fractions $\bar{w}_{1}$ and $\bar{w}_{2}$ of mineral bone and bone marrow, respectively, for the slice $S$, region $R_{\mathrm{F}}$, noise levels $L$ and 8th iteration.

\begin{tabular}{|c|c|c|c|c|c|}
\hline$S$ & $L$ & $\bar{w}_{1}$ & $\begin{array}{r}\delta\left(\bar{w}_{1}\right) \\
(\%) \\
\end{array}$ & $\bar{w}_{2}$ & $\begin{array}{r}\delta\left(\bar{w}_{2}\right) \\
(\%) \\
\end{array}$ \\
\hline A & 0 & 0.201 & -1.1 & 0.799 & 0.3 \\
\hline A & 1 & 0.199 & -2.1 & 0.801 & 0.5 \\
\hline A & 2 & 0.198 & -2.2 & 0.802 & 0.6 \\
\hline B & 0 & 0.201 & -0.9 & 0.799 & 0.2 \\
\hline B & 1 & 0.201 & -0.9 & 0.799 & 0.2 \\
\hline B & 2 & 0.198 & -2.2 & 0.802 & 0.6 \\
\hline $\mathrm{C}$ & 0 & 0.200 & -1.6 & 0.800 & 0.4 \\
\hline $\mathrm{C}$ & 1 & 0.199 & -1.8 & 0.801 & 0.5 \\
\hline $\mathrm{C}$ & 2 & 0.195 & -3.8 & 0.805 & 1.0 \\
\hline
\end{tabular}

